\title{
Acoustic Echo Cancellation in the Presence of Microphone Arrays
}

\author{
submitted by \\ Trevor Burton, B.Sc.E \\ A thesis submitted to \\ the Faculty of Graduate Studies and Research \\ in partial fulfillment of \\ the requirements for the degree of
}

\section{Master of Applied Science}

\author{
Ottawa-Carleton Institute for Electrical and Computer Engineering \\ Faculty of Engineering \\ Department of Systems and Computer Engineering \\ Carleton University \\ Ottawa, Ontario, Canada K1S 5B6 \\ December 2004 \\ (C) Copyright 2004, Trevor Burton
}




$\begin{array}{ll}\begin{array}{l}\text { Library and } \\ \text { Archives Canada }\end{array} & \begin{array}{l}\text { Bibliothèque et } \\ \text { Archives Canada }\end{array} \\ \begin{array}{l}\text { Published Heritage } \\ \text { Branch }\end{array} & \begin{array}{l}\text { Direction du } \\ \text { Patrimoine de l'édition }\end{array} \\ \begin{array}{l}\text { 395 Wellington Street } \\ \text { Ottawa ON K1A ON4 }\end{array} & \begin{array}{l}\text { 395, rue Wellington } \\ \text { Ottawa ON K1A ON4 } \\ \text { Canada }\end{array}\end{array}$

Your file Votre référence

ISBN: 0-494-00736-2

Our file Notre référence

ISBN: 0-494-00736-2

NOTICE:

The author has granted a nonexclusive license allowing Library and Archives Canada to reproduce, publish, archive, preserve, conserve, communicate to the public by telecommunication or on the Internet, loan, distribute and sell theses worldwide, for commercial or noncommercial purposes, in microform, paper, electronic and/or any other formats.

The author retains copyright ownership and moral rights in this thesis. Neither the thesis nor substantial extracts from it may be printed or otherwise reproduced without the author's permission.
AVIS:

L'auteur a accordé une licence non exclusive permettant à la Bibliothèque et Archives Canada de reproduire, publier, archiver, sauvegarder, conserver, transmettre au public par télécommunication ou par l'Internet, prêter, distribuer et vendre des thèses partout dans le monde, à des fins commerciales ou autres, sur support microforme, papier, électronique et/ou autres formats.

L'auteur conserve la propriété du droit d'auteur et des droits moraux qui protège cette thèse. $\mathrm{Ni}$ la thèse ni des extraits substantiels de celle-ci ne doivent être imprimés ou autrement reproduits sans son autorisation.
In compliance with the Canadian

Privacy Act some supporting forms may have been removed from this thesis.

While these forms may be included in the document page count, their removal does not represent any loss of content from the thesis.
Conformément à la loi canadienne sur la protection de la vie privée, quelques formulaires secondaires ont été enlevés de cette thèse.

Bien que ces formulaires aient inclus dans la pagination, il n'y aura aucun contenu manquant.

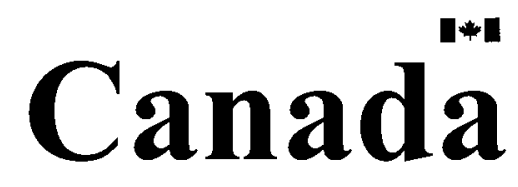




\begin{abstract}
Hands-free communication can be applied to many practical applications such as teleconferencing, video conferencing, voice over Internet Protocol (VoIP) conferencing, and mobile telephony to make communicating more convenient and safer. In order to make sure that a high quality conversation exists between parties the hands-free system requires a microphone array beamformer, to reduce background noise, as well as an acoustic echo canceller to prevent the far-end signal from being transmitted back to the far-end.
\end{abstract}

This thesis proposes a new structure for combining acoustic echo cancellation and microphone array beamforming that outperforms current structures under changing conditions within the hands-free environment. Simulations have shown that by taking into consideration the dynamic behaviour of the hands-free environment, the proposed structure outperforms current combined structures by up to approximately $2 \mathrm{~dB}$ on average during changing acoustical environment conditions. 


\section{ACKNOWLEDGEMENTS}

I would like to sincerely thank my supervisor, Dr. Rafik A. Goubran, for his invaluable guidance, encouragement, and enthusiasm during the course of this work. In addition, I would like to also thank Mitel Networks for their constructive comments and suggestions that arose during the Carleton-Mitel research interactions meetings. I would also like to thank our DSP lab technologist, Chris Welsh, for his assistance with the experimental setup and data acquisition portion of this thesis.

Also, I would like to thank Communications and Information Technology Ontario (CITO), the Natural Sciences and Engineering Research Council of Canada (NSERC), and Carleton University for their financial support during this research. 


\section{TABLE OF CONTENTS}

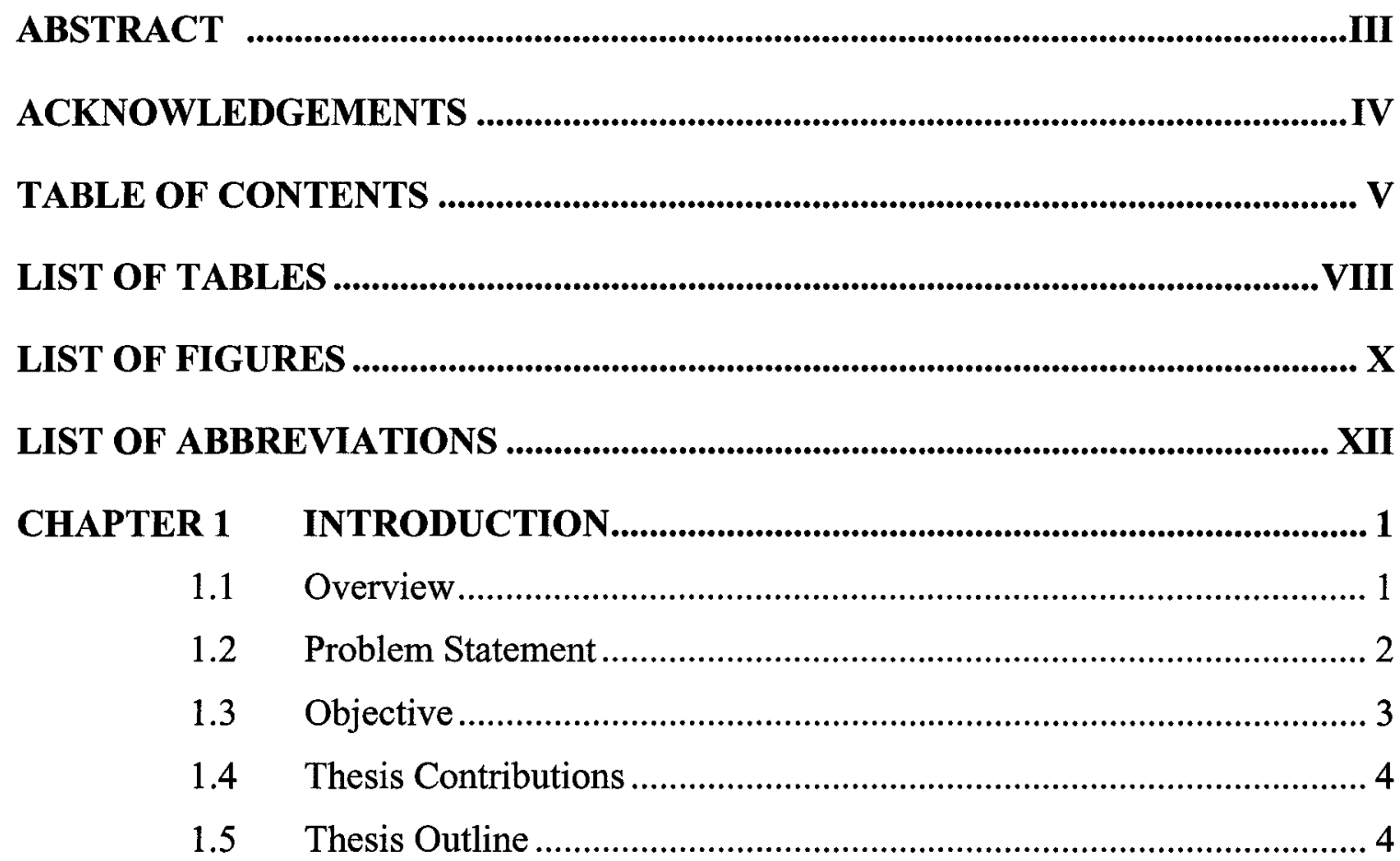

CHAPTER 2 BACKGROUND REVIEW .................................................... 6

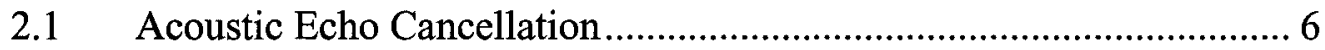

2.2 Microphone Array Beamforming ….............................................. 11

2.3 Combined Microphone Array Beamforming and Acoustic Echo Cancellation Approaches ............................................................. 15

$2.4 \quad$ Literature Review Summary .................................................... 18

CHAPTER 3 CURRENT AND PROPOSED STRUCTURES ............................. 20

3.1 Single Microphone Structure ........................................................ 20

3.2 Microphone Array Beamformer (MABF) Followed By Single Acoustic Echo Canceller (AEC) Structure ........................................ 21

3.3 Multi Channel AEC Followed By MABF Structure......................... 23

3.4 Proposed Multi Channel AEC Followed By MABF Followed By Single Channel AEC Structure ....................................................... 25

3.5 Underlying Algorithms Used in Implementing Structures ................ 28

3.5.1 Normalized Least Mean Squares (NLMS) Algorithm........ 29

3.5.2 Sector Based Delay-and-Sum Beamforming ...................... 30 


\section{CHAPTER 4 EXPERIMENTAL SETUP AND DATA ACQUISITION.............. 33}

$4.1 \quad$ Room Impulse Response (IR) Measurements....................................... 33

4.2 Loudspeaker-Room-Microphone (LRM) IR Recording Setup............ 33

4.3 Determining LRM IRs from Recorded Data ....................................... 35

$4.4 \quad$ Acquired LRM IRs ....................................................................... 36

4.4.1 Typical Office LRM IR ……………………………........... 37

4.4.2 Acquired LRM IRs under Changing Room Configurations 38

CHAPTER 5 SIMULATION OF STRUCTURES UNDER FIXED AND CHANGING SECTOR BASED BEAMFORMING CONDITIONS

5.1 Simulation Environment and Methodology ........................................... 43

5.2 Simulation of Structures under Stationary Room and Fixed BF

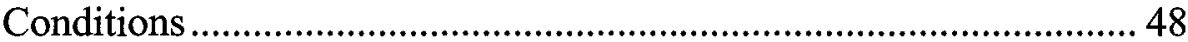

5.2.1 Stationary Room and Fixed BF Simulation Results ............. 49

5.2.1.1 Simulation Results under Increasing Front-End AEC Taps..

5.2.1.2 Simulation Results under Increasing Front-End AEC Taps Towards Full Echo Path Modeling .. 51

5.2.1.3 Simulation Results under Increasing Front-End AEC Step Size 54

5.2.1.4 Simulation Results under Increasing Tail-End AEC Step Size 56

5.2.1.5 Stationary Room and Fixed BF Simulation Results Summary 58

5.3 Simulation of Structures under Changing Sector Based BF Conditions 59

5.3.1 Changing Sector Based BF Simulation Results 60

5.3.1.1 Simulation Results under Increasing Front-End AEC Taps 60

5.3.1.2 Simulation Results under Increasing Front-End AEC Taps Towards Full Echo Path Modeling .. 63

5.3.1.3 Simulation Results under Increasing Front-End AEC Step Size 66

5.3.1.4 Simulation Results under Increasing Tail-End AEC Step Size 68 
5.3.1.5 Changing Sector Based BF Simulation Results

Summary

CHAPTER 6 SIMULATION OF STRUCTURES UNDER CHANGING ROOM CONDITIONS.......................................................................... 73

6.1 Simulation of Structures under Changing Room and Fixed BF

Conditions 73

6.1.1 Simulation Results under an Artificially Changing Room Environment....................................................................... 74

6.1.1.1 Simulation Results under Increasing Front-End AEC Taps.......................................................... 75

6.1.1.2 Simulation Results under Increasing Front-End AEC Taps Towards Full Echo Path Modeling .. 79

6.1.1.3 Simulation Results under Increasing Front-End AEC Step Size 82

6.1.1.4 Simulation Results under Increasing Tail-End AEC Step Size 85

6.1.1.5 Artificially Changing Room Environment Simulation Results Summary

6.1.2 Simulation Results under a Real Changing Room Environment 91

6.1.2.1 Simulation Results under Increasing Front-End AEC Taps.

6.1.2.2 Simulation Results under Increasing Front-End AEC Taps Towards Full Echo Path Modeling .. 94

6.1.2.3 Simulation Results under Increasing Front-End AEC Step Size

6.1.2.4 Simulation Results under Increasing Tail-End AEC Step Size 100

6.1.2.5 Real Changing Room Environment Simulation Results Summary 103

CHAPTER 7 CONCLUSIONS 106

7.1 Summary of Results..................................................................... 106

7.2 Recommendations for Future Research ............................................... 109

REFERENCES 


\section{LIST OF TABLES}

TABLE 5.1 Average Steady State ERLE under Stationary Room Conditions with Increasing Front-End AEC Taps for Proposed Structure

TABLE 5.2 Average Steady State ERLE under Stationary Room Conditions with Increasing Front-End AEC Taps Towards Full Echo Path Modeling for Proposed Structure

TABLE 5.3 Average Steady State ERLE under Stationary Room Conditions with Increasing Front-End AEC Step Sizes for Proposed Structure. 55

TABLE 5.4 Average Steady State ERLE under Stationary Room Conditions with Increasing Tail-End AEC Step Sizes for Proposed Structure

TABLE 5.5 Average ERLE Performance under Changing Sector Based BF Conditions with Increasing Front-End AEC Taps for Proposed Structure

TABLE 5.6 Average ERLE Performance under Changing Sector Based BF Conditions with Increasing Front-End AEC Taps Towards Full Echo Path Modeling for Proposed Structure.

TABLE 5.7 Average ERLE Performance under Changing Sector Based BF Conditions with Increasing Front-End AEC Step Sizes for Proposed

Structure

TABLE 5.8 Average ERLE Performance under Changing Sector Based BF Conditions with Increasing Tail-End AEC Step Sizes for Proposed Structure.

TABLE 6.1 Average ERLE Performance under Artificially Changing Room Conditions with Increasing Front-End AEC Taps for Proposed Structure

TABLE 6.2 Average ERLE Performance under Artificially Changing Room Conditions with Increasing Front-End AEC Taps Towards Full Echo Path Modeling for Proposed Structure.

TABLE 6.3 Average ERLE Performance under Artificially Changing Room Conditions with Increasing Front-End AEC Step Sizes for Proposed Structure.

TABLE 6.4 Average ERLE Performance under Artificially Changing Room Conditions with Increasing Tail-End AEC Step Sizes for Proposed Structure.

TABLE 6.5 Average ERLE Performance under Real Changing Room Conditions with Increasing Front-End AEC Taps for Proposed Structure

TABLE 6.6 Average ERLE Performance under Real Changing Room Conditions with Increasing Front-End AEC Taps Towards Full Echo Path Modeling for Proposed Structure 96 
TABLE 6.7 Average ERLE Performance under Real Changing Room Conditions with Increasing Front-End AEC Step Sizes for Proposed Structure ..... 99

TABLE 6.8 Average ERLE Performance under Real Changing Room Conditions with Increasing Tail-End AEC Step Sizes for Proposed Structure...... 102 


\section{LIST OF FIGURES}

FIGURE 1.1 Single Microphone Hands-free System ........................................... 1

FIGURE 2.1 Half-Duplex Hands-Free System ..................................................... 7

FIGURE 2.2 Single Channel Acoustic Echo Cancellation Process ........................... 8

FIGURE 2.3 Microphone Array Beamformer in a Hands-Free System .................... 12

FIGURE 2.4 Generic Approaches for Combining Microphone Array Beamforming and Acoustic Echo Cancellation .......................................................... 16

FIGURE 3.1 Single-microphone Hands-free Communication Structure.................... 20

FIGURE 3.2 Microphone Array Beamformer Followed By a Single Acoustic Echo Canceller Structure............................................................................ 22

FIGURE 3.3 Multi Channel Acoustic Echo Canceller Followed By a Microphone Array Beamformer Structure ......................................................... 24

FIGURE 3.4 Compromise AEC-BF-AEC Structure ............................................. 27

FIGURE 3.5 Sector Based Approach to Beamforming ........................................... 31

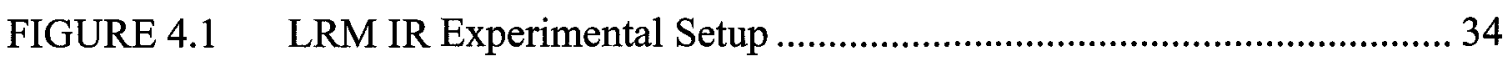

FIGURE 4.2 Determining LRM IRs Using NLMS Adaptive Filters........................ 35

FIGURE 4.3 Typical Conference Room LRM IRs................................................ 37

FIGURE 4.4 LRM IR Changing Room Condition Experimental Setup.................... 39

FIGURE 4.5 LRM IRs Obtained Under Room Configuration One.......................... 40

FIGURE 4.6 LRM IRs Obtained Under Room Configuration Two .......................... 41

FIGURE 5.1 Blank MATLAB GUI Screen Shot................................................... 44

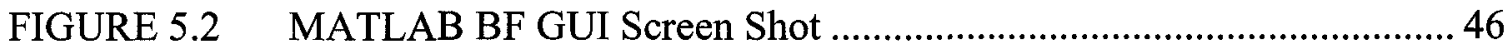

FIGURE 5.3 ERLE Results under Stationary Room Conditions with Increasing FrontEnd AEC Taps for Proposed Structure ............................................. 50

FIGURE 5.4 ERLE Results under Stationary Room Conditions with Increasing FrontEnd AEC Taps Towards Full Echo Path Modeling for Proposed

Structure .................................................................................... 52

FIGURE 5.5 ERLE Results under Stationary Room Conditions with Increasing FrontEnd AEC Step Sizes for Proposed Structure 54

FIGURE 5.6 ERLE Results under Stationary Room Conditions with Increasing TailEnd AEC Step Sizes for Proposed Structure ........................................ 56

FIGURE 5.7 ERLE Results under Changing Sector Based BF Conditions with Increasing Front-End AEC Taps for Proposed Structure 61 
FIGURE 5.8 ERLE Results under Changing Sector Based BF Conditions with Increasing Front-End AEC Taps Towards Full Echo Path Modeling for Proposed Structure 64

FIGURE 5.9 ERLE Results under Changing Sector Based BF Conditions with Increasing Front-End AEC Step Sizes for Proposed Structure.. 66

FIGURE 5.10 ERLE Results under Changing Sector Based BF Conditions with Increasing Tail-End AEC Step Sizes for Proposed Structure.

FIGURE 6.1 Modified LRM IRs used to Artificially Simulate Changing Room Acoustical Conditions 75

FIGURE 6.2 ERLE Results under Artificially Changing Room Conditions with Increasing Front-End AEC Taps for Proposed Structure . 76

FIGURE 6.3 ERLE Results under Artificially Changing Room Conditions with Increasing Front-End AEC Taps Towards Full Echo Path Modeling for Proposed Structure

FIGURE 6.4 ERLE Results under Artificially Changing Room Conditions with Increasing Front-End AEC Step Sizes for Proposed Structure.

FIGURE 6.5 ERLE Results under Artificially Changing Room Conditions with Increasing Tail-End AEC Step Sizes for Proposed Structure 86

FIGURE 6.6 ERLE Results under Real Changing Room Conditions with Increasing Front-End AEC Taps for Proposed Structure 92

FIGURE 6.7 ERLE Results under Real Changing Room Conditions with Increasing Front-End AEC Taps Towards Full Echo Path Modeling for Proposed Structure

FIGURE 6.8 ERLE Results under Real Changing Room Conditions with Increasing Front-End AEC Step Sizes for Proposed Structure

FIGURE 6.9 ERLE Results under Real Changing Room Conditions with Increasing Tail-End AEC Step Sizes for Proposed Structure. 101 


\section{LIST OF ABBREVIATIONS}

$\begin{array}{ll}\text { AEC } & \text { Acoustic Echo Canceller } \\ \text { BF } & \text { Beamformer } \\ \text { DSP } & \text { Digital Signal Processing } \\ \text { ERLE } & \text { Echo Return Loss Enhancement } \\ \text { FAP } & \text { Fast Affine Projection } \\ \text { GUI } & \text { Graphical User Interface } \\ \text { IR } & \text { Impulse Response } \\ \text { LMS } & \text { Least Mean Squares } \\ \text { LRM } & \text { Loudspeaker-Room-Microphone } \\ \text { MABF } & \text { Microphone Array Beamformer } \\ \text { MSE } & \text { Mean Square Error } \\ \text { NLMS } & \text { Normalized Least Mean Squares } \\ \text { RLS } & \text { Recursive Least Squares } \\ \text { WGN } & \text { White Gaussian Noise } \\ \text { VoIP } & \text { Voice over Internet Protocol }\end{array}$

xii 


\section{CHAPTER 1 INTRODUCTION}

\subsection{Overview}

The classical approach to removing acoustical echo, caused by reverberant room environments, from a full-duplex hands-free communication system requires an acoustic echo canceller (AEC). The AEC is generally implemented via an adaptive filter which is an area of study that has been rigorously researched over many years [1], [2], [3], [4]. Figure 1.1 shows one half of the setup of an AEC for a single-microphone hands-free communication system. The AEC setup is replicated at the far-end of the communication system.

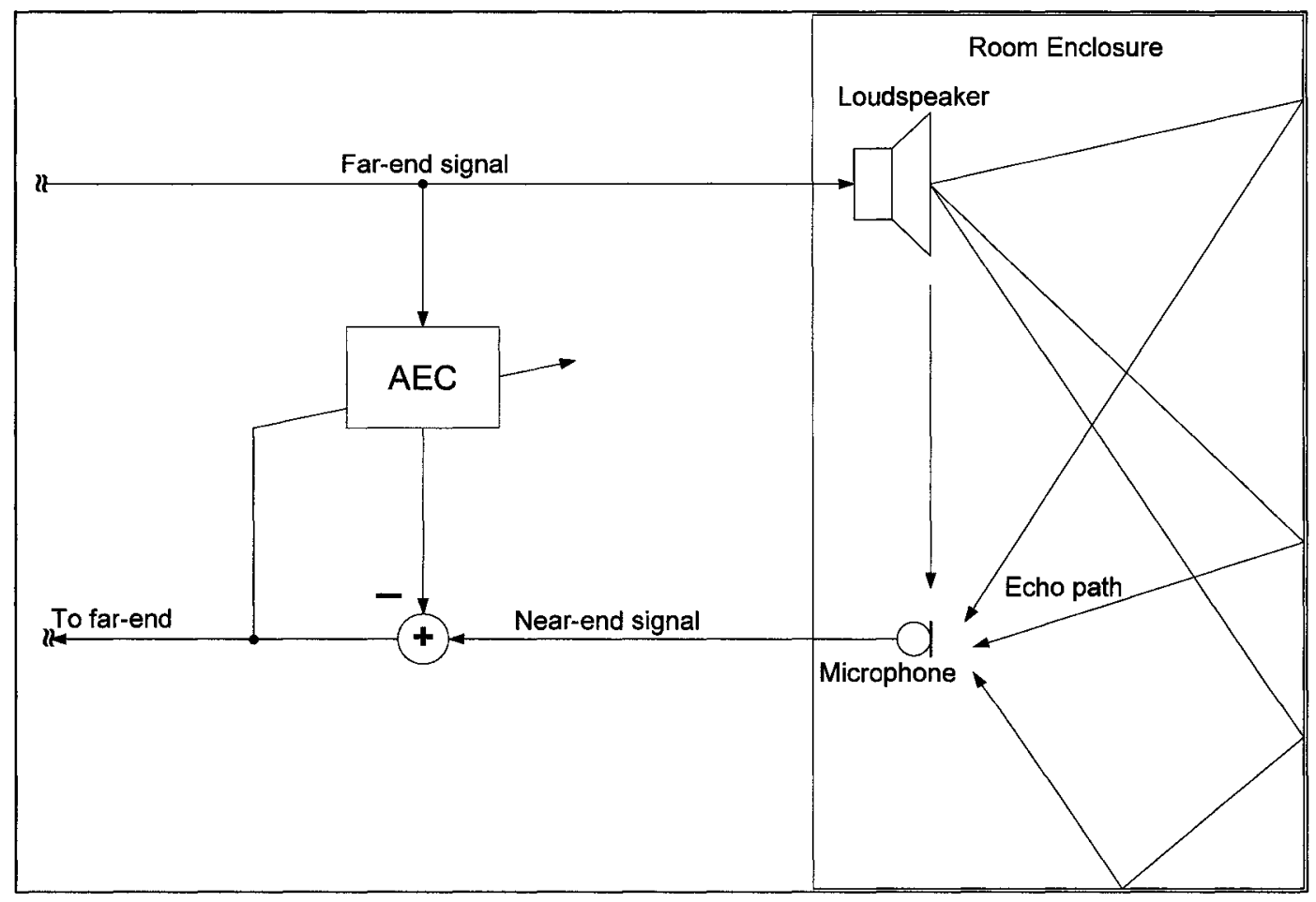

FIGURE 1.1 Single Microphone Hands-Free System 
The single-microphone approach to acoustic echo cancellation is limited in its effectiveness to suppress echo by background noise sources, non-stationarities in the acoustical environment, and system non-linearities [5]. In order to mitigate the decrease in performance of the single-microphone AEC system, multi-microphone approaches have been proposed [6], [7], [8], [9], [10], [11]. These methods involve combining microphone array beamforming and acoustic echo cancellation techniques.

While all of the multi-microphone approaches strive to guarantee a superior hands-free conversation exists between parties, the manner in which this is achieved varies. Some techniques are implemented in the time-domain while others in the frequency-domain, with different underlying beamforming and acoustic echo cancellation approaches. In all cases there is generally a trade-off between the amount of achievable echo cancellation and complexity of the strategies used to attain it.

\subsection{Problem Statement}

The optimal manner to combine the techniques of microphone array beamforming and acoustic echo cancellation in hands-free communication systems is the subject of much research. An overview of strategies for combining the two techniques is given by Kellermann in [12] and [13]. The findings of these works suggest that for a multimicrophone hands-free communication setup, a structure with an AEC per microphone input followed by a single beamformer (BF) is not practical due to its high computational complexity, especially for a large number of microphones. 
An opposite structure is also discussed, where a single AEC follows a microphone array BF. This structure has reduced complexity, due to the single AEC, but the single AEC has to model not only the acoustic echo path but also any time-variations of the BF [12]. This becomes increasingly difficult if an adaptive $\mathrm{BF}$ is employed. One way to alleviate the problems introduced by an adaptive $\mathrm{BF}$ is to use a fixed $\mathrm{BF}$ instead.

Thus, the main focus of this thesis is to develop a combined AEC-BF structure for handsfree communication systems that acts as a compromise between the aforementioned two structures in terms of achievable acoustic echo cancellation, complexity, and robustness to time-variations in the acoustical and beamforming environments.

\subsection{Objective}

The main objectives of this thesis are as follows:

- To develop a compromise AEC-BF structure

- Verification of the compromise AEC-BF structure under stationary conditions

- Verification of the compromise AEC-BF structure under time-varying acoustical environments

- Verification of the compromise AEC-BF structure under time-varying beamforming conditions

As a result of meeting the above objectives the main contribution of this thesis will be the development of a compromise AEC-BF structure that achieves equal or better acoustic echo cancellation performance than current structures, especially under non-stationary 
conditions. Ideally the low to moderate complexity compromise structure will be suitable to be implemented in a real-world hands-free communication system.

\subsection{Thesis Contributions}

The following are the main contributions of this thesis work:

- Proposition of a new structure combining acoustic echo cancellation and microphone array beamforming that outperforms existing structures and takes into account the dynamic behaviour of the unknown environment

- Comparison of the proposed structure to existing structures under changing acoustical and beamforming conditions to demonstrate that the proposed structure outperforms the current structures under these conditions

- Verification that the proposed structure outperforms the current structures under the above conditions using real experimental data

- Investigation of the dynamic behaviour of typical office room acoustics and suggestion of a varying transfer function model to track this behaviour

- Design of a MATLAB based GUI used to facilitate simulating all of the hands-free communication system structures investigated in this thesis

\subsection{Thesis Outline}

The remainder of this thesis is organized as follows. Chapter 2 provides a background review on acoustic echo cancellation, beamforming, and the combination of the two techniques. Chapter 3 discusses current combined AEC-BF structures along with the 
proposed compromise structure, where the advantages and disadvantages of each are highlighted.

Experimental setup and data acquisition is discussed in Chapter 4. Specifically the procedure for acquiring loudspeaker-room-microphone (LRM) impulse responses (IR), to be used in the computer simulations of the hands-free structures, is outlined.

In Chapter 5 the simulation environment and methodology are discussed. Also, the compromise $\mathrm{AEC}-\mathrm{BF}$ structure is compared to current combined structures along with a single microphone structure via computer simulation. The structures are simulated using measured LRM IRs to generate the microphone signals and compared under fixed and time-varying beamforming conditions. Discussions of results are also presented. Chapter 6 follows the same ideology as Chapter 5 except the structures are compared under time-varying acoustical conditions with fixed beamforming.

Finally, conclusions are presented in Chapter 7 along with recommendations for future research, and a summary of contributions. 


\section{CHAPTER 2 BACKGROUND REVIEW}

\subsection{Acoustic Echo Cancellation}

One of the original methods to achieve acoustic echo cancellation in a hands-free system involved a switching mechanism to determine the active talker [5]. This method is inherently a half-duplex system since the switching mechanism allows voice to be transmitted in only one direction at a time which leads to an unnatural conversation. Also, clipping and chopping of words can occur as a result of the finite time required to switch from one voice path to the other [5]. A typical half-duplex hands-free communication setup is shown in Figure 2.1 and is adapted from [5]. 


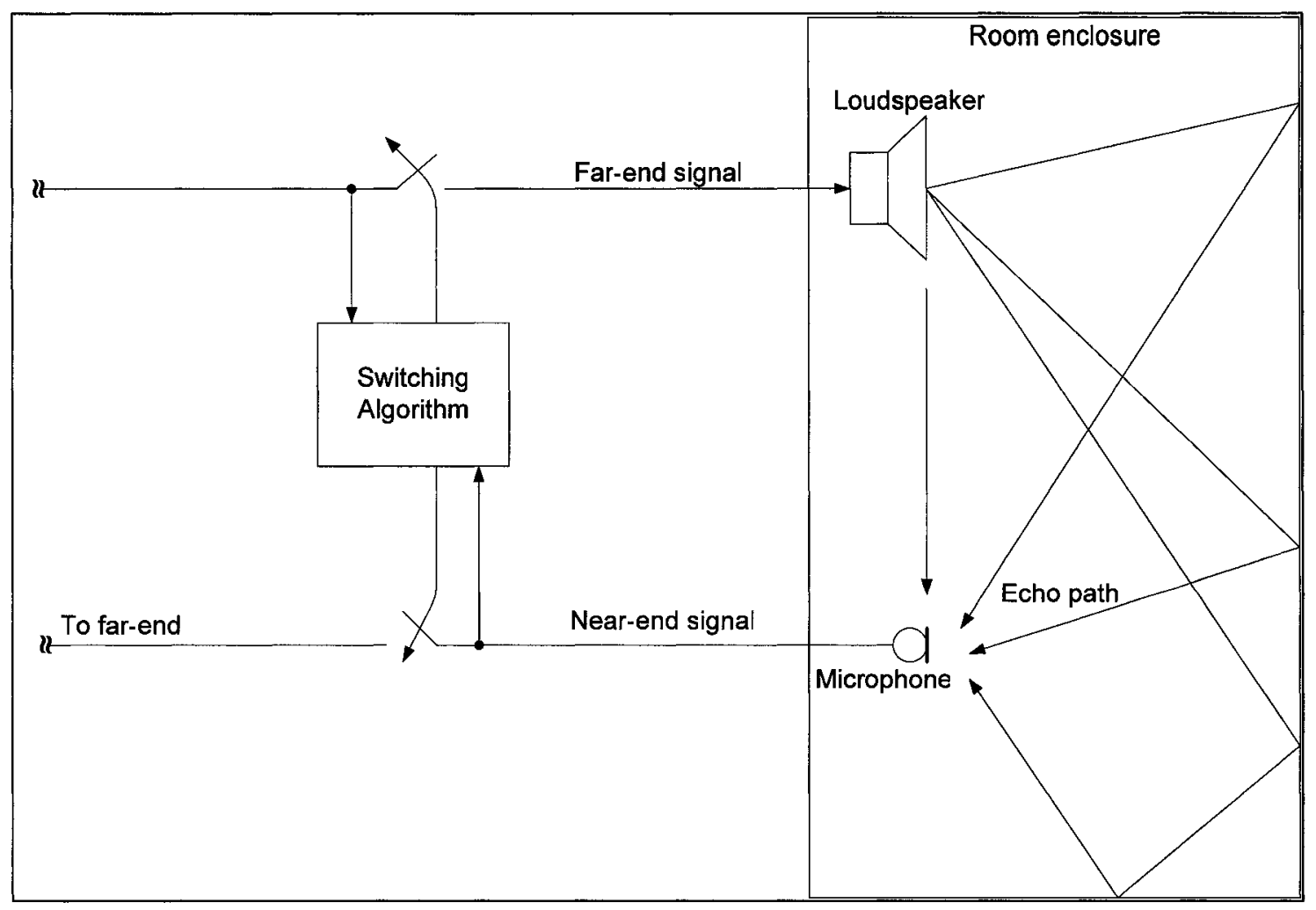

FIGURE 2.1 Half-Duplex Hands-Free System

Current methods for implementing acoustic echo cancellation in a full-duplex hands-free communication system, as shown in Figure 1.1, employ adaptive filtering techniques and has been actively researched over the years [1], [2], [3], [4]. The goal of the adaptive filter is to estimate the transfer function of the loudspeaker-room-microphone (LRM) environment and thereby remove the undesired echo from being transmitted back to the far-end. Figure 2.2 outlines the single channel acoustic echo cancellation process and is adapted from [14]. It should be noted that the adaptive filter of the acoustic echo canceller only adapts when the local talker is quiet in order to avoid cancellation of the local talker signal. 


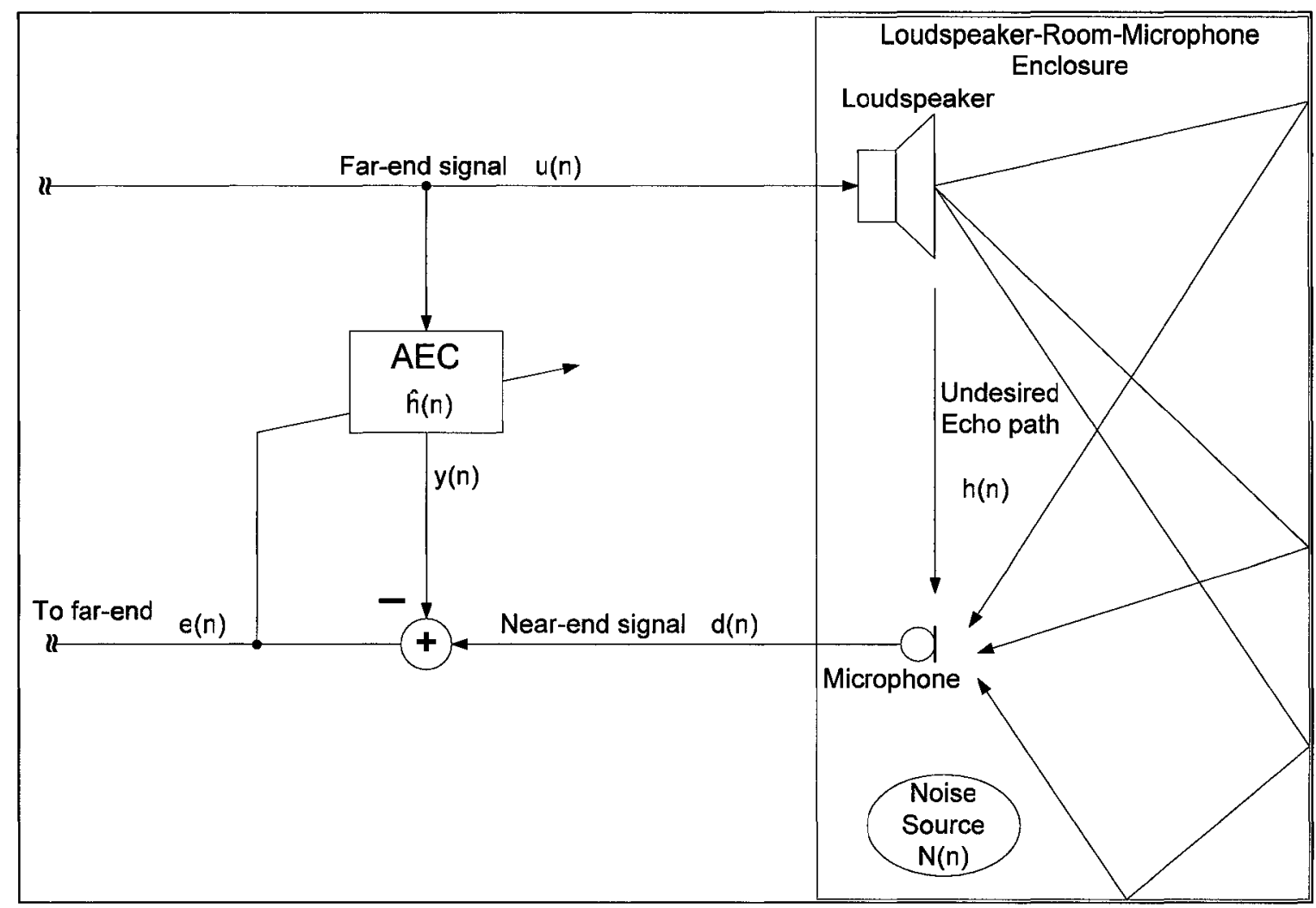

FIGURE 2.2 Single Channel Acoustic Echo Cancellation Process

Mathematically the acoustic echo cancellation problem depicted in Figure 2.2 can be modeled as follows, where any system nonlinearities are ignored [14]:

$$
\begin{aligned}
e(n) & =d(n)-y(n)+N(n) \\
& =\boldsymbol{u}(n) * \boldsymbol{h}(n)-\boldsymbol{u}(n) * \hat{\boldsymbol{h}}(n)+N(n)
\end{aligned}
$$

Where:

$e(n)$ is the echo cancelled signal sent back to the far-end

$\boldsymbol{u}(n)$ is the far-end signal vector

$d(n)$ is the near-end microphone signal

$y(n)$ is the output signal from the AEC 
$N(n)$ is an additive noise signal

$\boldsymbol{h}(n)$ is the LRM impulse response vector

$\hat{\boldsymbol{h}}(n)$ is the estimated LRM impulse response vector

The most extensively used adaptive filtering algorithm in acoustic echo cancellation is the least mean squares (LMS) algorithm, or one of its variants. This is due to it simple implementation, low complexity, and performance robustness [14], [15]. However, the main drawback of the LMS algorithm is its slow convergence rate for input signals with a large eigenvalue spread such as speech [16], [17]. A theoretical investigation of the LMS algorithm is presented in [18] and [19].

In order to overcome the relatively slow convergence rate of the LMS type adaptive filtering algorithms many alternative adaptive filtering algorithms have been introduced over the years. One set of adaptive filtering algorithms that improve upon the slow convergence speed of the LMS algorithms are the recursive least squares (RLS) algorithms [20]. Although the RLS algorithm converges faster than the LMS algorithms it does so at the expense of much greater computational complexity. As a result the RLS algorithm itself it not generally used in acoustic echo cancellation systems. In order to reduce the complexity of the RLS algorithm fast versions of the algorithm have been developed over the years [21], [22], [23], [24]. The fast RLS adaptive algorithms can still be too complex for use in acoustic echo cancellation systems. This is due to the very long adaptive filters that may be required to sufficiently model the LRM impulse response (echo path) that can range in the thousands of taps. 
Frequency domain adaptive filtering and subband adaptive filtering are two other techniques that have been applied to acoustic echo cancellation [16], [25], [26], [27], [28], [29], [30]. Both of these techniques aim to reduce the complexity and increase the rate of convergence compared to the general LMS algorithm. However, both of these methods have their drawbacks. In the case of frequency domain adaptive filtering and subband adaptive filtering an inherent delay is introduced to the resulting echo cancelled signal that can lead to unnatural full-duplex hands-free conversations. Also, the subband adaptive filtering approach may suffer from aliasing in the output signal due to the down sampling operations.

Another adaptive filtering algorithm that has been applied to acoustic echo cancellation is the fast affine projection (FAP) algorithm [14], [31]. The FAP algorithm has complexity similar to the LMS algorithm, while providing convergence speed similar to the RLS type algorithms. Another important characteristic of the FAP algorithm is that does not introduce any delays unlike the frequency domain and subband adaptive filtering techniques mentioned above. However, the FAP algorithm may not be suitable for acoustic echo cancellation where extremely long adaptive filters are required to accurately model the LRM echo path since this will result in high computational complexity.

Variable step size LMS adaptive filtering algorithms [32], [33], [34] are another set of algorithms aimed at improving the convergence rate compared to the standard LMS 
algorithm. These variable step size algorithms achieve faster convergence while maintaining the same steady state performance as standard LMS with little increase in computational complexity. Also, variable step size LMS type algorithms have an additional advantage of improved tracking performance in non-stationary environments compared to the general LMS algorithm. Again, the variable step size LMS algorithms may not be suitable for acoustic echo cancellation systems where very long adaptive filters are required to effectively model the acoustic echo path.

\subsection{Microphone Array Beamforming}

The purpose of a microphone array is to capture a desired signal from a specific location while attenuating interference signals such as noise [35]. Thus, microphone arrays lend themselves readily for use in a hands-free communication system. The way in which the individual microphone signals are processed to acquire the desired signal while attenuating interference signals is called beamforming. Beamforming methods have been vigorously researched over the years. An excellent overview of beamforming is given by Van Veen and Buckley in [36]. A block diagram of a general microphone array beamformer in a hands-free communication system environment is shown in Figure 2.3 and is adapted from [37] and [36]. 


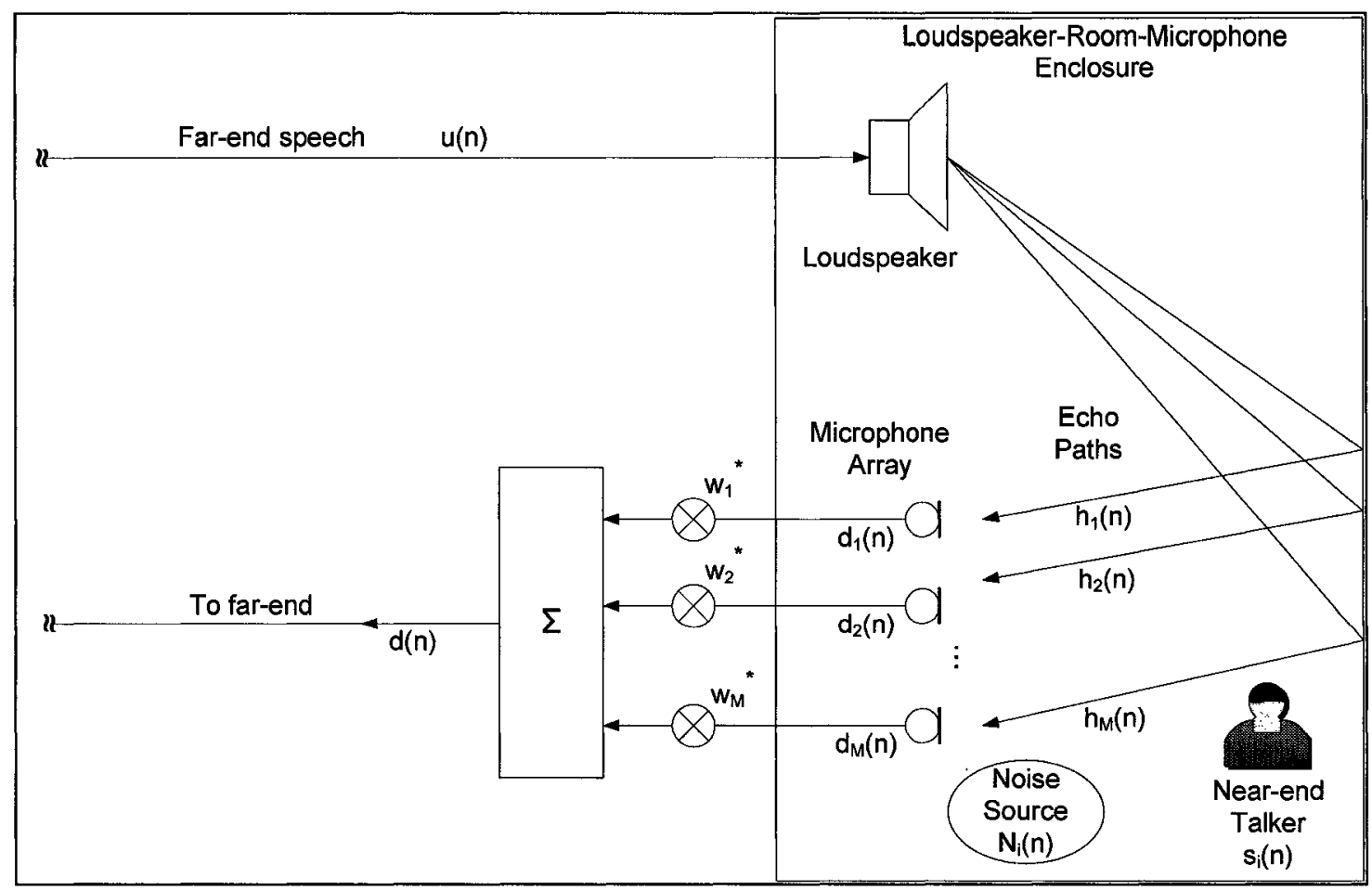

FIGURE 2.3 Microphone Array Beamformer in a Hands-Free System

The signals acquired by each microphone in the microphone array shown in Figure 2.3 can be modeled, ignoring any system nonlinearities, as follows:

$$
d_{i}(n)=s_{i}(n)+h_{i}(n) * u(n)+N_{i}(n), \quad i=1, \ldots, M
$$

Where:

$M$ is the total number of microphones in the array

$s_{i}(n)$ is the near-end talker signal seen by microphone $i$

$\boldsymbol{u}(n)$ is the far-end signal vector

$d_{i}(n)$ is the near-end microphone signal acquired by microphone $i$

$d(n)$ is the beamformed microphone signals

$N_{i}(n)$ is an additive noise signal for microphone $i$ 
$\boldsymbol{h}_{i}(n)$ is the LRM impulse response vector seen by microphone $i$

$w_{i}^{*}$ is a complex weighting factor applied to microphone signal $d_{i}(n)$

All of the various beamforming approaches, whether they are data independent or statistically optimum, are implemented using either adaptive or fixed techniques [37]. The goal of a data independent beamformer is to provide a fixed optimum response, in terms of minimizing the squared error between the actual and desired responses, independent of the sensor data or statistics for all signal scenarios [36]. A more flexible beamformer is a statistically optimal one where the statistics of the sensor data are used to drive the beamformer to produce an optimized output. The statistically optimum beamformer tries to nullify the signals arriving from directions other that the desired one in an attempt to maximize the signal to noise ratio at the beamformer output [36].

The simplest beamforming technique is the fixed delay-and-sum beamformer. The basic idea behind delay-and-sum beamforming is to constructively reinforce a desired signal emitting from a specific location while attenuating interference signals [38]. This is achieved by delay compensating the acquired microphone signals, to offset the propagation delays between the reference microphone and the other microphones in the array, and then summing the resulting signals [39]. By replacing the complex weights in Figure 2.3 with fixed delays, based on the desired source location, for each microphone signal a delay-and-sum beamformer is realized. The main advantages of fixed beamforming techniques are ease of implementation and low computational complexity. The output of a delay-and-sum beamformer is described by the following equation: 


$$
d(n)=\left[\frac{1}{M} \sum_{i=1}^{M} d_{i}\left(n-\tau_{i}\right)\right]
$$

Where the signal and variable definitions are the same as above for Equation 2 with the exception of:

$\tau_{i}$ is a fixed delay applied to microphone signal $d_{i}(n)$

Although fixed beamforming techniques are relatively simple to implement they provide a fixed response which may not be desirable, or yield sufficient signal to noise ratio gain, in all beamforming situations. Thus, a statistically optimum beamformer may be a more advantageous approach. Several classical statistically optimum beamforming methods, such as the multiple sidelobe canceller and the linearly constrained minimum variance beamformer, are presented and compared in [36]. Since the statistics of the array sensor data may not be know and can vary over time adaptive algorithms are generally used in statistically optimum beamforming to overcome this. However, employing adaptive techniques in a statistically optimum beamformer adds considerable computational complexity; they can be slow to converge, and they can introduce desired signal cancellation due to reverberation [37]. In order to alleviate some of the drawbacks of adaptive beamforming partially adaptive beamformers have been introduced [36], [40], [41]. Partially adaptive beamformers offset the disadvantages of their fully adaptive counterparts by using fewer of the available adaptive degrees of freedom [11]. This results in a reduction in computational complexity as well as a faster converged response. 
However, interference cancellation performance is sacrificed as fewer of the adaptive degrees of freedom are used in the design of a partially adaptive beamformer [40].

Another method to reduce some of the disadvantages of fully adaptive beamforming is achieved via subband beamforming techniques [42], [43]. A subband beamformer has the advantages of improved interference suppression, quicker convergence, and reduced computational complexity compared to the general fully adaptive beamformer [43]. However, like all subband filtering techniques, subband beamforming suffers from signal delay. Also, aliasing between different subbands can lead to problems in the reconstructed signal. However the problems caused by aliasing can be mitigated by oversampling in the subbands [43].

\subsection{Combined Microphone Array Beamforming and Acoustic Echo Cancellation Approaches}

In many hands-free communication systems, especially those operating in noisy environments, a single microphone acoustic echo cancellation approach is often inadequate for ensuring a high quality full-duplex conversation exists between parties. Thus, many specific approaches have been developed that combine microphone array beamforming methods with acoustic echo cancellation techniques in order to achieve sufficient acoustic echo cancellation and noise reduction in adverse hands-free communication systems [6], [8], [9], [44], [10], [45], [46], [47], [11]. In [12] Kellermann outlines strategies for combining acoustic echo cancellation and adaptive beamforming microphone arrays. He discusses two generic approaches for combining the two 
techniques, which are shown in Figure 2.4 below and adapted from [12]. The structures are generic in the sense that they are applicable regardless of the way in which the AEC is implemented, be it a fullband, transform, or subband domain implementation, and regardless of the underlying adaptation algorithms.

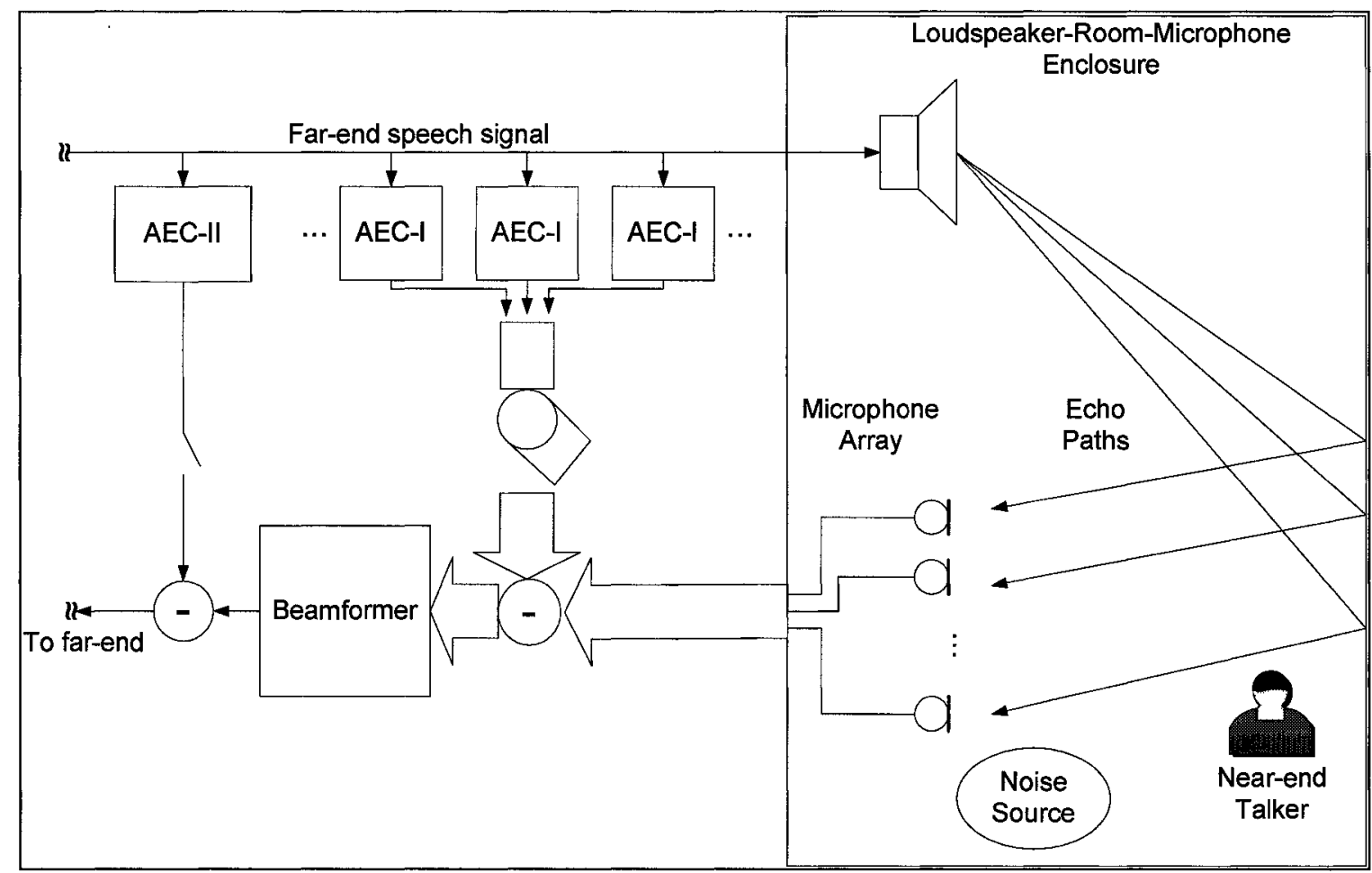

FIGURE 2.4 Generic Approaches for Combining Microphone Array Beamforming and Acoustic Echo Cancellation

The first generic structure that Kellermann talks about involves an acoustic echo canceller applied directly to each acquired microphone signal (AEC-I) followed by an adaptive beamformer. Since the adaptive beamformer operates on the outputs of the AECs the AECs are not disturbed by any time variations in the beamformer and thus each AEC can be implemented in the same fashion as in a single microphone AEC situation. However, a major drawback of this structure is that for each microphone in the array an 
AEC is required resulting in a very large complexity structure, especially when long adaptive filters are required to accurately model each LRM echo path. As a result this structure is generally not implemented for real time hands-free communication systems.

The second generic structure that Kellermann discusses in his paper employs an adaptive beamformer with a single AEC (AEC-II) operating on the beamformed output signal. This structure is much less computationally complex than the previous one as only a single $\mathrm{AEC}$ is required. However, because the AEC acts on the beamformer output it has to include the beamformer in its model of the acoustic echo path. Also, any time variations in the beamformer need to be tracked by the AEC. Since the AEC only adapts when the local talker is silent it will not be able to track changes in the beamformer output when the beamformer changes its parameters to focus on a different active talker. As a result of the time variance of the adaptive beamformer the amount of acoustic echo cancellation is hindered and therefore this structure is also discouraged from being used in a real time hands-free communication system.

In order to alleviate the large computational complexity of the first structure and the time variance of the adaptive beamformer in the second structure Kellermann proposes two alternate efficient structures in [12]. In order to avoid the time varying effects of an adaptive beamformer each structure employs a beamformer with a time invariant stage followed by a time varying stage. The time invariant state of each structure is implemented via a fixed beamformer that operates directly on the microphone signals. The fixed beamformer produces a minimum number of output signals to be acoustically 
echo cancelled. Since fewer AECs are required for both structures a computational savings is realized compared to the first generic structure discussed above. Also, because the time varying part of the beamformer is separated from the AECs the AECs are not hindered by any time variations of the beamformer. Although these new structures are more complex than the second generic structure they minimize the problems caused by the time varying nature of the adaptive beamformer.

\subsection{Literature Review Summary}

The review of past work in the area of acoustic echo cancellation applied to hands-free communication systems has shown that incorporating microphone array beamforming techniques can improve the quality of the hands-free communication compared to the single microphone approach. The strategies for combining the microphone array beamformer with the acoustic echo canceller, outlined by Kellermann in [12], discuss two generic hands-free communication structures. The first structure requires multiple acoustic echo cancellers, one per microphone input, followed by a beamformer. This structure has an inherently large computational complexity and is therefore impractical for real-time implementation. The second combined structure uses a beamformer applied directly to the microphone inputs followed by a single acoustic echo canceller. This structure has a much lower complexity than the previous structure but the acoustic echo canceller in this case has to track any time variations of the beamformer, which will disrupt the performance of the hands-free system. 
This thesis investigates the two combined structures discussed above and proposes a compromise combined structure to minimize the drawbacks of the aforementioned structures. 


\section{CHAPTER 3 CURRENT AND PROPOSED STRUCTURES}

\subsection{Single Microphone Structure}

The single microphone hands-free communication structure is the simplest of the structures compared in this thesis. Although the single microphone system is simple to implement and has a low computational complexity, in some cases it may not be able to attain sufficient acoustic echo cancellation performance, especially in environments where there are strong sources of background noise. The single microphone hands-free structure is shown in Figure 2.2 but is reproduced in Figure 3.1 below for convenience.

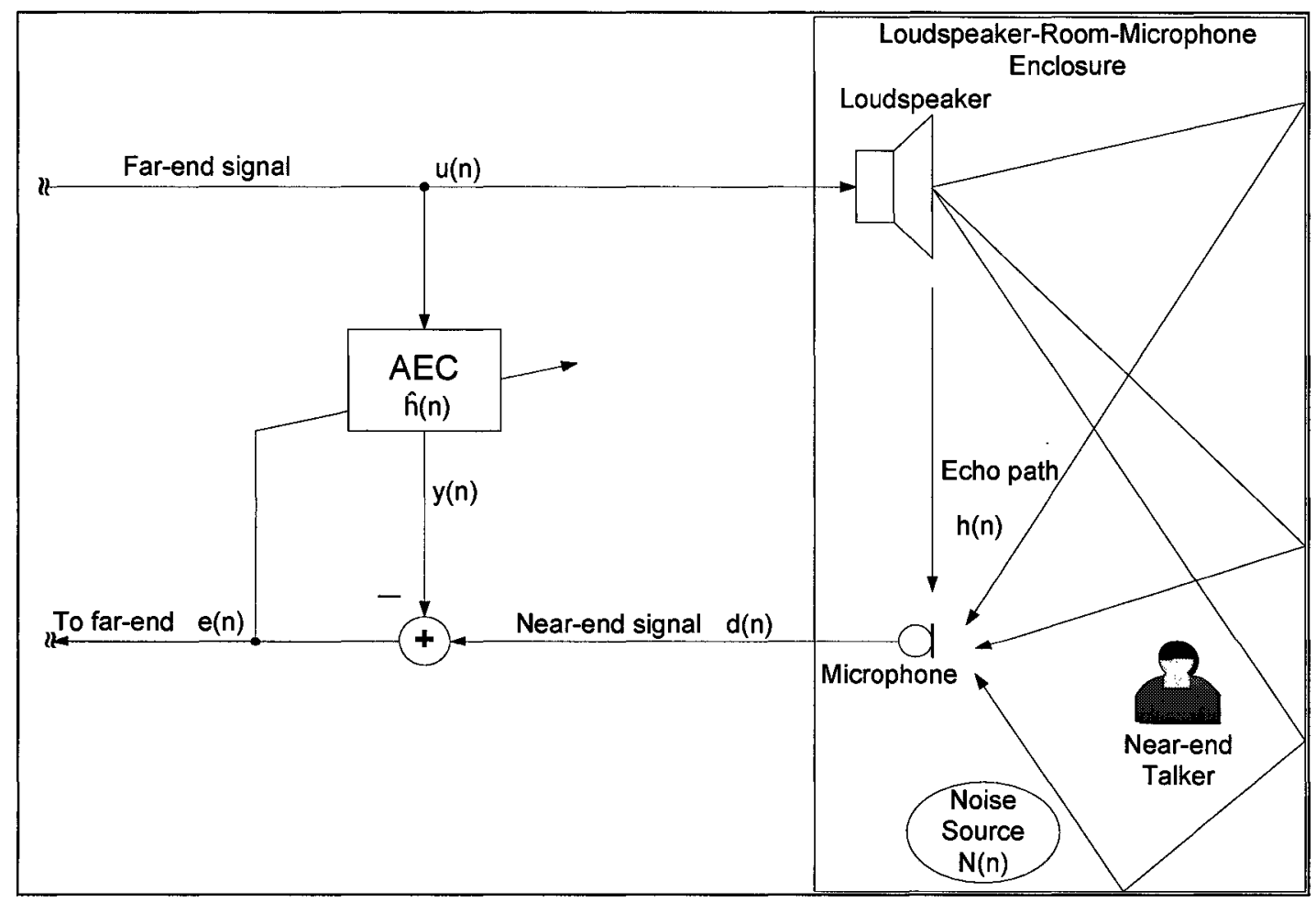

FIGURE 3.1 Single Microphone Hands-Free Communication Structure 
It is expected that all of the combined microphone array beamformer and acoustic echo cancellation structures discussed below will outperform the single microphone structure at the cost of increased complexity. The signal names and equation relating them for the single microphone structure are the same as for Figure 2.2 in Chapter 2.

\subsection{Microphone Array Beamformer (MABF) Followed By Single Acoustic Echo Canceller (AEC) Structure}

The microphone array beamformer followed by a single acoustic echo canceller structure (BF-AEC) should attain better acoustic echo cancellation and noise reduction results than the single microphone structure due to the incorporation of the beamformer. However, an increase in computational complexity will be realized in order to achieve these improved results. The BF-AEC structure is shown in Figure 3.2 below. 


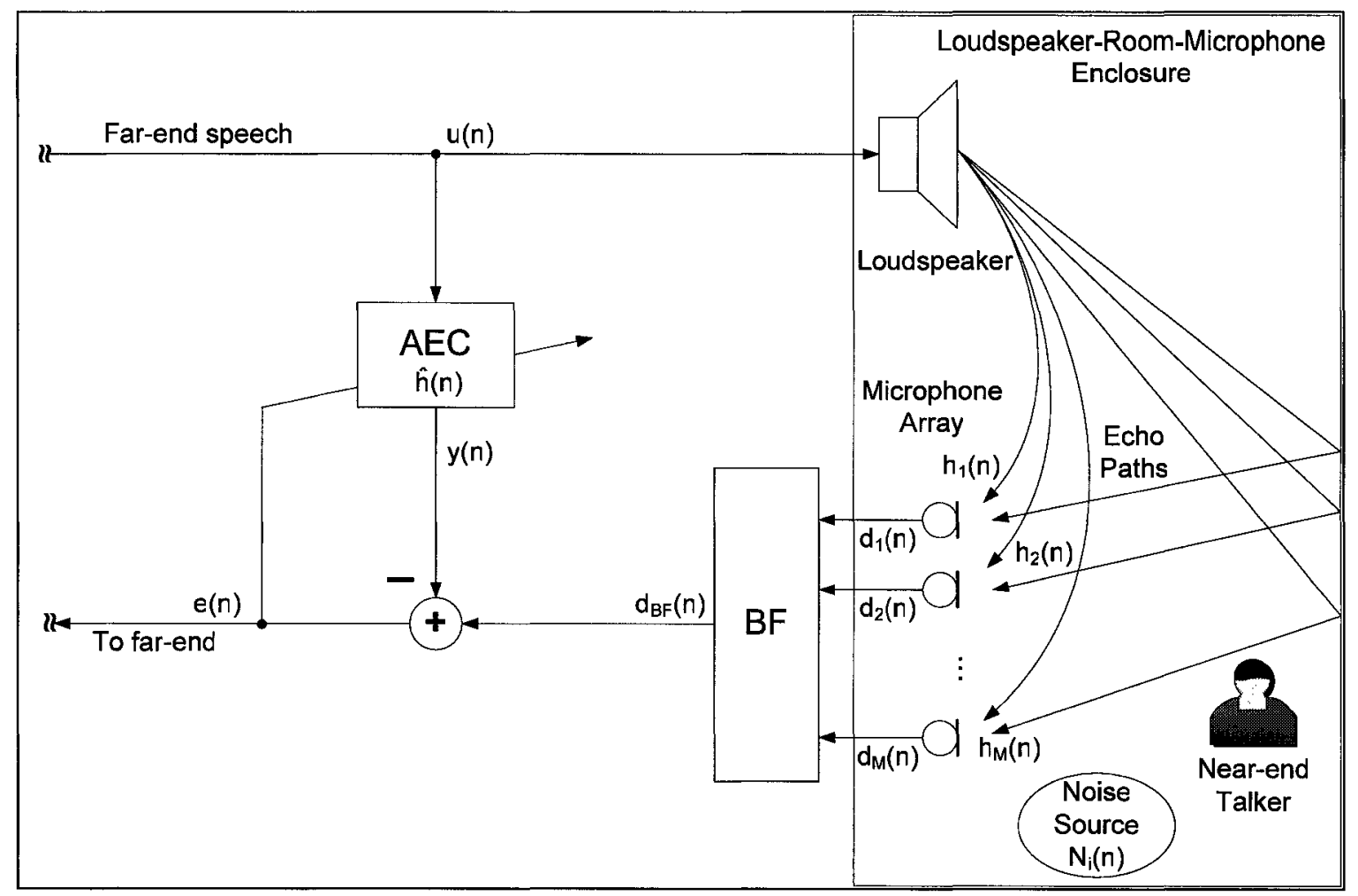

FIGURE 3.2 Microphone Array Beamformer Followed By a Single Acoustic Echo Canceller Structure

A mathematical description of the above BF-AEC structure, assuming a linear only model for the LRM impulse response and quiet local talker, is given below.

$$
\begin{aligned}
d_{i}(n) & =\boldsymbol{h}_{i}(n)^{*} \boldsymbol{u}(n)+N_{i}(n), \quad i=1, \ldots, M \\
e(n) & =d_{B F}(n)-y(n) \\
& =\left[\frac{1}{M} \sum_{i=1}^{M} d_{i}\left(n-\tau_{i}\right)\right]-\boldsymbol{u}(n) * \hat{\boldsymbol{h}}(n)
\end{aligned}
$$

Where:

$e(n)$ is the echo cancelled and noise reduced signal sent back to the far-end $\boldsymbol{u}(n)$ is an $N$-by-1 far-end signal vector 
$d_{B F}(n)$ is the beamformed microphone signals

$y(n)$ is the output signal from the $\mathrm{AEC}$

$\hat{\boldsymbol{h}}(n)$ is the $N$-by-1 estimated LRM impulse response vector

$\boldsymbol{h}_{i}(n)$ is the $N$-by-1 LRM impulse response vector seen by microphone $i$

$M$ is the total number of microphones in the array

$d_{i}(n)$ is the near-end microphone signal acquired by microphone $i$

$N_{i}(n)$ is an additive noise signal for microphone $i$

$\tau_{i}$ is a fixed delay applied to microphone signal $d_{i}(n)$

$N$ is the number of adaptive filter taps

This structure has the added complexity of the beamformer compared to the single microphone structure but should produce better echo cancellation results. However, when the beamformer changes its delay parameters the AEC will have to track these changes and degradation in the echo cancellation performance will result.

\subsection{Multi Channel AEC Followed By MABF Structure}

The multi channel acoustic echo canceller followed by microphone array beamformer structure (AEC-BF) should also attain better acoustic echo cancellation and noise reduction results than the single microphone structure due to the incorporation of the beamformer. However, a severe increase in computational complexity will be realized in order to achieve these improved results as a result of the required AEC per microphone input. The AEC-BF structure is shown in Figure 3.3 below. 


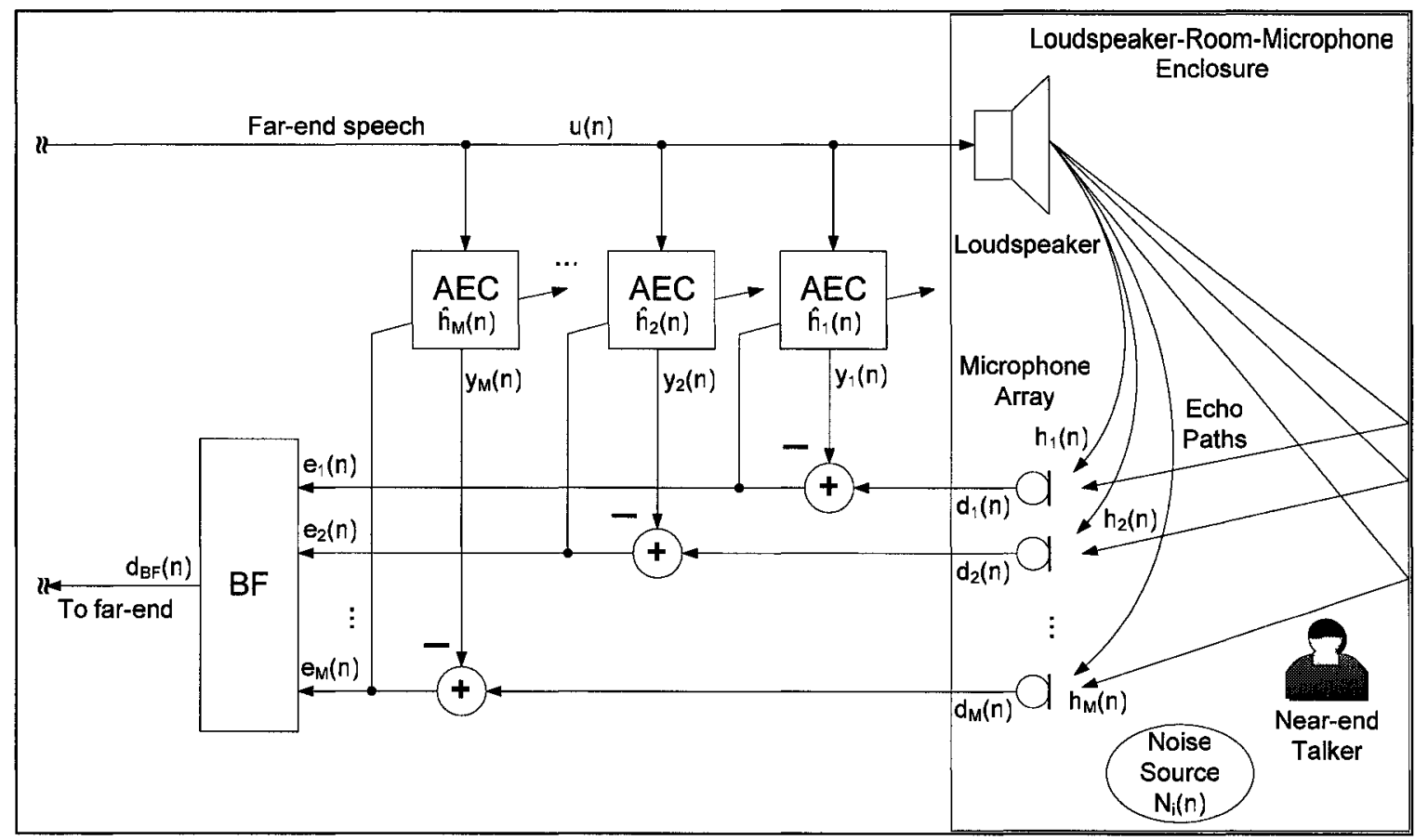

FIGURE 3.3 Multi Channel Acoustic Echo Canceller Followed By a Microphone Array Beamformer Structure

A mathematical description of the above structure is given below. Again it is assumed that each LRM impulse response is linear and any system nonlinearities are ignored. Also, it is assumed the local talker is in a non-talk period.

$$
\begin{gathered}
e_{i}(n)=d_{i}(n)-y_{i}(n) \\
=\boldsymbol{h}_{i}(n) * u(n)+N_{i}(n)-\boldsymbol{u}(n) * \hat{\boldsymbol{h}}_{i}(n), \quad i=1, \ldots, M \\
d_{B F}(n)=\frac{1}{M} \sum_{i=1}^{M} e_{i}\left(n-\tau_{i}\right)
\end{gathered}
$$

Where the signal definitions are as in Section 3.2 with the exception of:

$e_{i}(n)$ is the echo cancelled signal for microphone $i$

$y_{i}(n)$ is the output signal from the AEC for microphone $i$ 
$d_{B F}(n)$ is the beamformed echo cancelled signal that is sent back to the far-end

This structure should produce better echo cancellation results than the single microphone system but at the cost of a severe increase in computational complexity due to the required AEC per microphone input. However, when the beamformer changes its delay parameters the AECs will not have to track these changes and degradation in the echo cancellation performance will not result as in the BF-AEC structure of Section 3.2.

\subsection{Proposed Multi Channel AEC Followed By MABF Followed By Single Channel AEC Structure}

Due to the decrease in echo cancellation performance caused by beamformer time variations in the BF-AEC structure and the large computational complexity requirements of the AEC-BF structure a compromise structure is proposed in this work. Ideally this structure will have a complexity similar to the BF-AEC structure and the immunity to beamformer time variations of the AEC-BF structure, while achieving equal or better echo cancellation performance under various operation conditions.

The proposed structure will employ an AEC for each microphone input but each AEC will be set to model only a small portion of the first part of each LRM impulse response. Because of the exponentially decaying nature of a typical LRM impulse response it is believed that by modeling only the first several taps each AEC will achieve significant acoustic echo cancellation at a small computational cost compared to the AEC-BF structure. Each partially echo cancelled microphone signal will then be beamformed 
using simple fixed delay-and-sum beamforming techniques (explained in Section 3.5). A final tail-end $\mathrm{AEC}$ will then operate on the beamformed signal to further remove acoustic echo by modeling the remainder of the echo path. Since the tail-end AEC models only the last part of the echo path it is expected that the overall echo cancellation performance of the structure will be affected less by any time variations of the delay-and-sum beamformer compared to the BF-AEC structure.

Since a change in the acoustical environment will cause a fluctuation in the LRM impulse response the AECs of all structures will have to track these fluctuations causing a decrease in echo cancellation performance. Since the front-end AECs of the proposed structure will model only the first part of each LRM echo path, where the majority of echo path fluctuation is expected to occur, these AECs will be able to track changes in each echo path faster due to the shorter adaptive filters used. As a result the compromise structure's overall acoustic echo cancellation performance should suffer less than the previously mention structures under changing room environments. The compromise AEC-BF-AEC structure is shown below in Figure 3.4. 


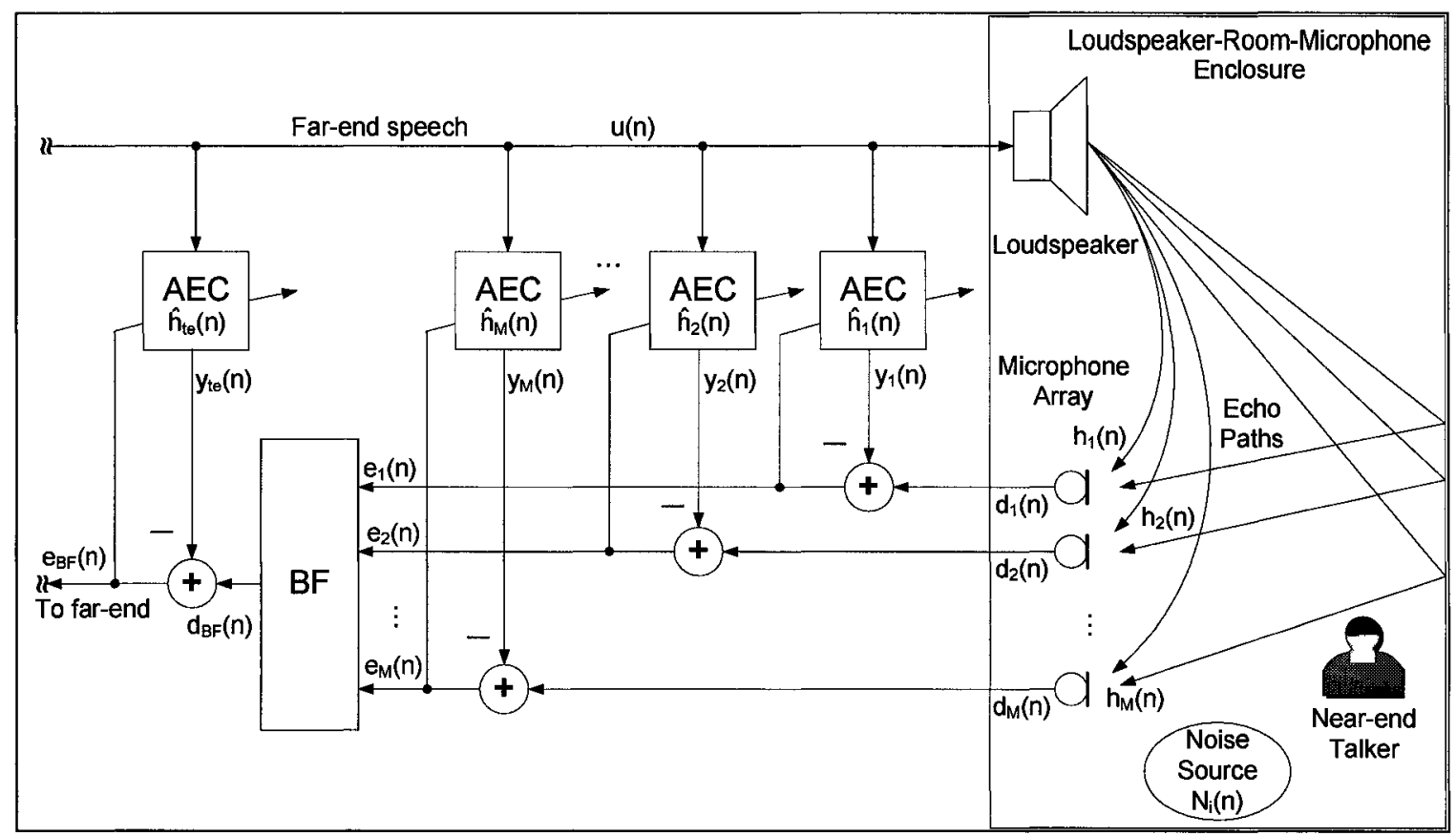

FIGURE 3.4 Compromise AEC-BF-AEC Structure

A mathematical description of the above structure is given below. Again it is assumed that each LRM impulse response is linear and any system nonlinearities are ignored. Also, it is assumed the local talker is in a non-talk period. The equations describing the compromise AEC-BF-AEC structure are the same as the AEC-BF structure of Section 3.3 with the addition of the following equation:

$$
\begin{aligned}
e_{B F}(n) & =d_{B F}(n)-y_{t e}(n) \\
& =\left[\frac{1}{M} \sum_{i=1}^{M} e_{i}\left(n-\tau_{i}\right)\right]-\boldsymbol{u}(n) * \hat{\boldsymbol{h}}_{t e}(n)
\end{aligned}
$$

Where the signal definitions are as in Section 3.3 with the exception of:

$Y_{t e}(n)$ is the output signal from the tail-end AEC

$\hat{\boldsymbol{h}}_{t e}(n)$ is the estimated LRM impulse response vector for the remainder of the echo path 
$d_{B F}(n)$ is the beamformed partially echo cancelled signal that is operated on by the tailend AEC

$e_{B F}(n)$ is the beamformed echo cancelled signal that is sent back to the far-end

\subsection{Underlying Algorithms Used in Implementing Structures}

The normalized least-mean square (NLMS) adaptive algorithm is used to implement the acoustic echo cancellation in all of the above structures. This algorithm was selected due to its low computational complexity, ease of implementation, and performance robustness [14], [15]. The beamforming method implemented in the above structures is sector based delay-and-sum beamforming. This beamforming method was chosen due to its simplicity and ease of implementation.

For the proposed structure, shown in Figure 3.4 above, the front-end AECs are adapted first followed by beamforming their outputs and then adaptation of the tail-end AEC. This sequential adaptation scheme is used in order to avoid adaptation conflicts that could arise if both the front and tail-end AECs were adapted simultaneously.

It should be noted that acoustic echo cancellation is only performed when local talkers are quiet and that it is assumed that a double-talk detector is providing the information on whether or not a local talker is active. The implementation of a double-talk detector is outside the scope of this thesis. Also, for computational complexity reasons linear adaptive filters are used in all the above structures and any nonlinear effects, as discussed 
in [5], are ignored. Nonlinear acoustic echo cancellation is discussed in [48-51] but is outside the scope of this thesis.

\subsubsection{Normalized Least Mean Squares (NLMS) Algorithm}

A good theoretical overview of the NLMS algorithm is given in [18] and [19] respectively. A summary of the NLMS algorithm, adapted from [19], and how it is applied in the above structures follows. In the acoustic echo cancellation blocks of all the above structures the error signal, $e(n)$, is determined by the following equation:

$$
e(n)=d(n)-\hat{\boldsymbol{h}}^{H}(n) * \boldsymbol{u}(n)
$$

Where:

$e(n)$ is the algorithm error signal at time $n$

$d(n)$ is the desired signal at time $n$

$\boldsymbol{u}(n)$ is an $N$-by-1 tap input vector an time $n$

$\hat{\boldsymbol{h}}(n)$ is the estimated adaptive filter taps at time $n$

$N$ is the number of adaptive filter taps

During each iteration of the NLMS algorithm Equation 9 is calculated and the adaptive filter taps are updated according to the following equation:

$$
\hat{\boldsymbol{h}}(n+1)=\hat{\boldsymbol{h}}(n)+\frac{\tilde{\mu}}{a+\|\boldsymbol{u}(n)\|^{2}} \boldsymbol{u}(n) e^{*}(n)
$$


Where:

$\tilde{\mu}$ is the adaptation step size constant

$a$ is a small positive constant

For the NLMS algorithm to converge in the mean square sense the following condition must be satisfied:

$$
0<\tilde{\mu}<2
$$

The constant $a$ helps to offset numerical difficulties that may occur when the value of the squared norm of the input vector is very small. Also, if no previous information of the adaptive filter taps is available then the taps are initialized to the zero vector at the beginning of the NLMS algorithm. The computational complexity of the NLMS algorithm is linear with respect to the number of adaptive filter taps, $\mathrm{N}$.

\subsubsection{Sector Based Delay-and-Sum Beamforming}

Sector based delay-and-sum beamforming involves dividing the area around the microphone array into individual segments and determining if there is an active talker in one of these segments. Figure 3.5 depicts this sector based approach for a linear microphone array. 


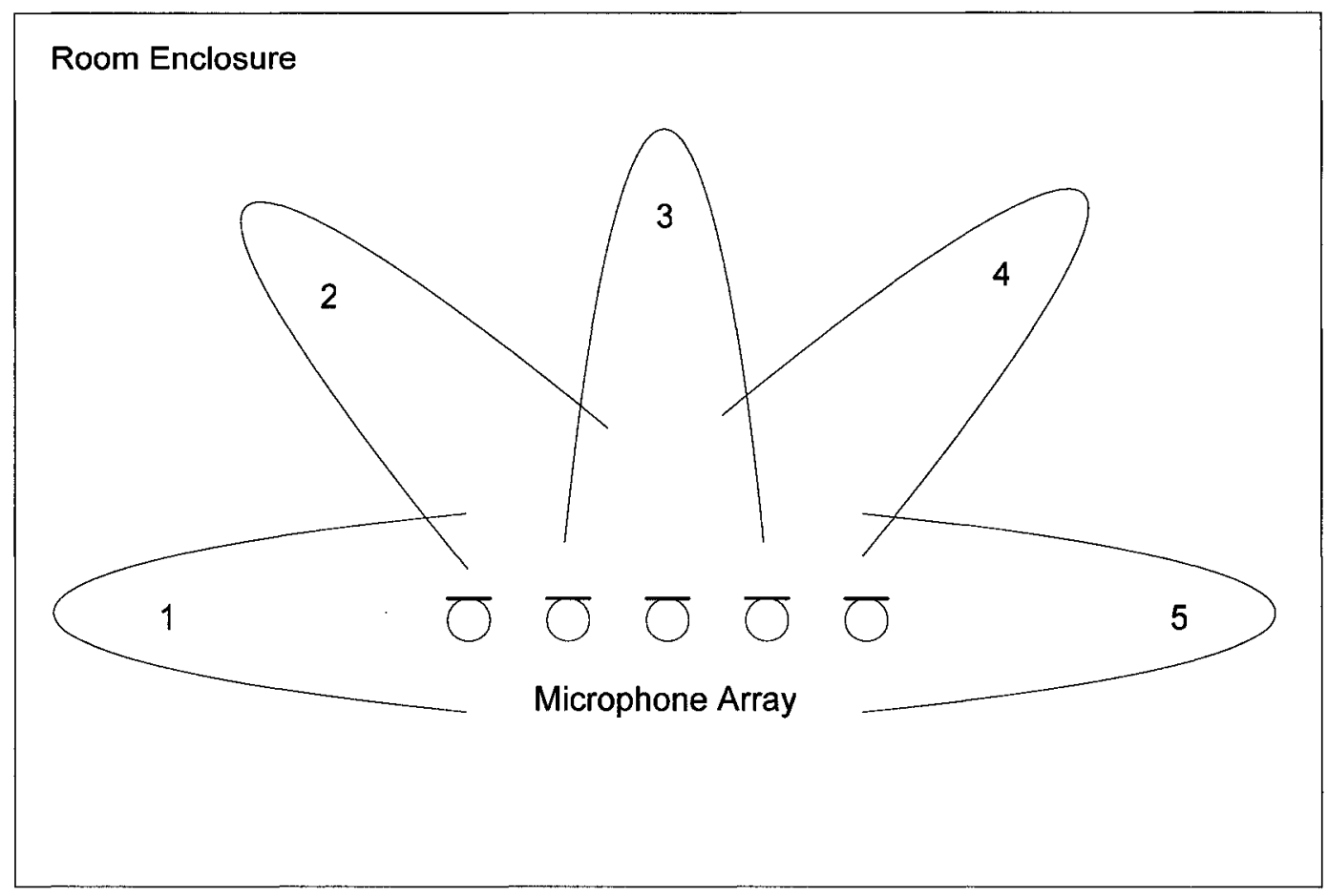

FIGURE 3.5 Sector Based Approach to Beamforming

The sectors are continually scanned to determine if there is an active talker in any one of them. Once the active sector is determined appropriate delays are applied to the microphone signals. The microphone signals are then averaged to produce the delay-andsum output. In this thesis the knowledge of the active sector is assumed and the details of how the active sector is determined are outside the scope of this project.

For a linear microphone array the relative delays between microphones can be determined using simple geometry assuming planar wave conditions. A more general approach to determine the relative delays between microphones for any configuration of a microphone array is to employ cross correlation methods. Determining the relative delays between microphones in the array using cross correlation involves recording a 
signal simultaneously with the microphone array and then cross correlating each individual microphone signal with the other microphone signals to determine the relative delays. 


\section{CHAPTER 4 EXPERIMENTAL SETUP AND DATA ACQUISITION}

\subsection{Room Impulse Response (IR) Measurements}

In order to carry out meaningful simulations of the structures compared in this thesis real world LRM IRs needed to be experimentally determined. This allowed artificial microphone signals to be created based upon real room acoustics.

The LRM IR experiments were performed in typical office rooms at Carleton University. The LRM IR experiment involved playing a reference noise signal through a loudspeaker into the room environment under test and recording the reproduced signal on each microphone of the microphone array. The recorded data was then post processed to determine the individual LRM IRs. The experimental recording setup and method for determining the individual LRM IRs are explained in the sections below.

\subsection{Loudspeaker-Room-Microphone (LRM) IR Recording Setup}

The experimental setup for acquiring individual LRM IRs is shown in Figure 4.1 below. In this setup a reference White Gaussian Noise (WGN) signal was reproduced through a loudspeaker into the room enclosure. The WGN signal was constructed from a sequence of normally distributed non-repeating random numbers spaced every $0.25 \mathrm{~ms}$, with zero mean and unit variance. The reproduced noise signal was then acquired by each 
microphone in the circular microphone array and recorded on a computer. The circular microphone array consisted of six identical equally spaced omnidirectional electret type microphones with a top mounted loudspeaker.

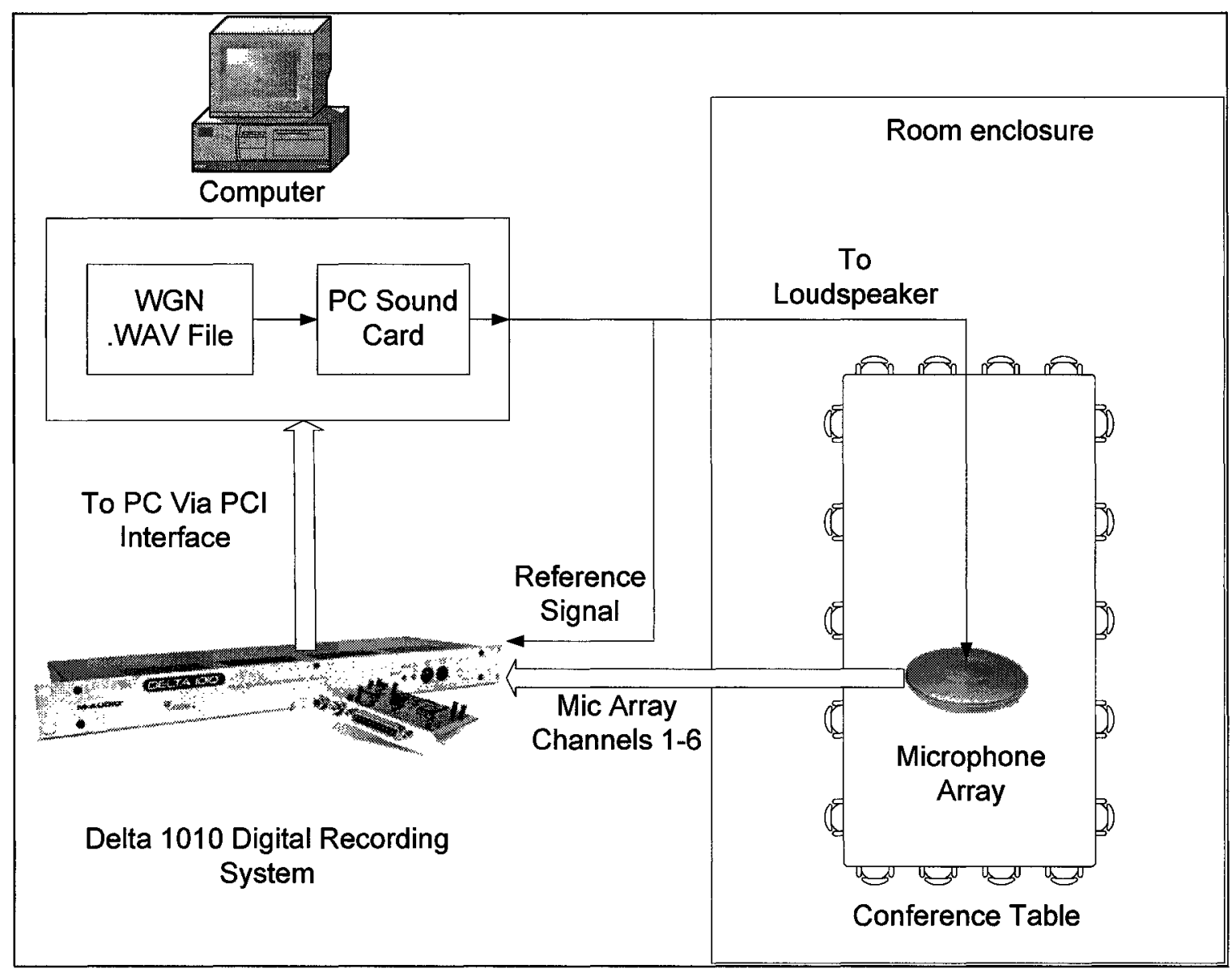

FIGURE 4.1 LRM IR Experimental Setup

The recording of the microphone signals and the reference noise signal was performed simultaneously at $8 \mathrm{kHz}$ by an M-AUDIO Delta 1010 digital recording system. The entire process of playing a reference WGN noise file from the computer to the loudspeaker and recording of the microphone and reference noise signals was carried out in the Adobe Audition audio editing environment. Once the microphone signals were 
recorded they were post processed using the NLMS algorithm to determine the individual LRM transfer functions as discussed in the following section.

\subsection{Determining LRM IRs from Recorded Data}

Determining the transfer function between a loudspeaker and each microphone of a microphone array in a room enclosure is a system identification problem that can be readily solved using adaptive filtering techniques. In this thesis the NLMS algorithm, as discussed in section 3.5.1, was used to determine the individual LRM IRs of the microphone array setup shown above. A block diagram of how the NLMS adaptive filters were used to determine the individual LRM IRs is shown in Figure 4.2 below.

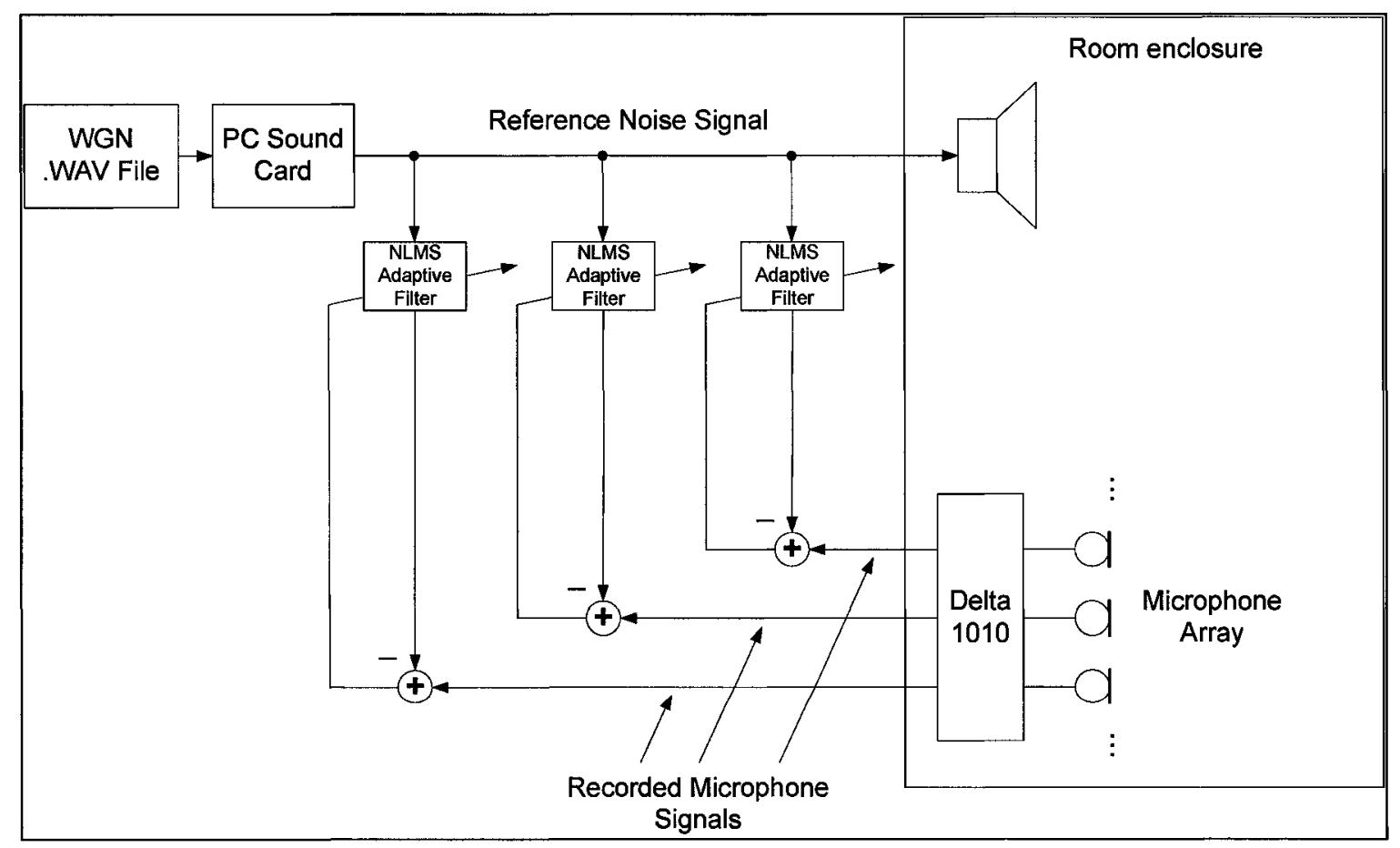

FIGURE 4.2 Determining LRM IRs Using NLMS Adaptive Filters 
In order to ensure a small adaptation error signal and thus a tight approximation to the true LRM transfer functions a small adaptation step size and large number of NLMS algorithm iterations were used. Again a linear model for the LRM IRs was assumed and any system nonlinearities were ignored.

Other methods for determining the LRM IRs include impulsive excitation and maximumlength sequence techniques as discussed in [52]. The adaptive filtering approach to determine the individual LRM transfer functions was selected as the method of choice in this thesis due to its simplicity, ease of implementation, and ability to provide an accurate linear model for each LRM IR.

\subsection{Acquired LRM IRs}

The following sections present typical LRM IR results obtained in different office rooms at Carleton University. The experiments were performed and LRM IRs obtained as outlined in the sections above. A circular prototype hands-free terminal, consisting of six equally spaced omni-directional microphones with a top mounted speaker, was used in the experiments. A thirty second WGN signal was used as the reference signal played through the loudspeaker. Once the microphone signals were recorded as described in the sections above, NLMS adaptive filtering was performed on each microphone signal over 20000 iterations using a step size of 0.1 . The NLMS adaptive filters were set to adapt to a 1000 tap linear model of each LRM IR in question. It was assumed that a 1000 tap linear model was sufficient to accurately describe each LRM transfer function. 


\subsubsection{Typical Office LRM IR}

The following set of recorded LRM IRs was obtained in a typical conference room at Carleton University (MC3033). The recording setup was as shown in Figure 4.1 above where the microphone array was placed in the center of the conference table and was unobstructed by any objects. The resulting set of obtained LRM IRs is shown in Figure 4.3 below.
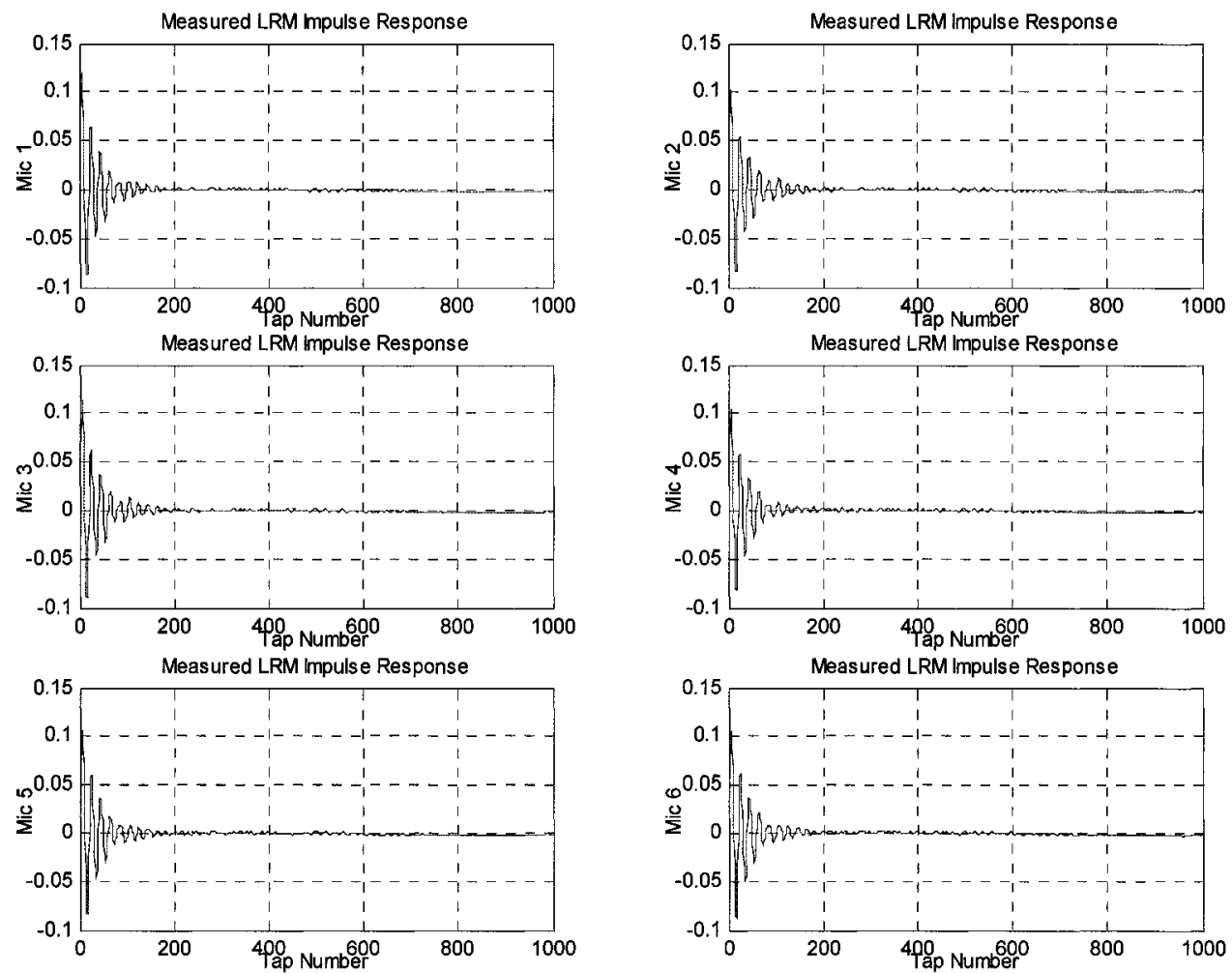

FIGURE 4.3 Typical Conference Room LRM IRs 
As expected the LRM IRs were all slightly different and decayed in an exponential manner. This set of typical conference room LRM IRs was used to create artificial microphone data in the stationary room simulations of Chapter 5.

\subsubsection{Acquired LRM IRs under Changing Room Configurations}

The next sets of measured LRM IRs were recorded under two different room configurations. The LRM IR recordings were performed in room MC6015 at Carleton University. The first set of recordings were performed with the microphone array located at an unobstructed position on a desk in the corner of the room as indicated by position one in Figure 4.4 below. The second set of recordings was acquired with the microphone array located in the corner of the desk where it was in close proximity to the back and side walls of the desk as well as to the overhead cabinets. This recording setup is indicated by position two in Figure 4.4 below. 


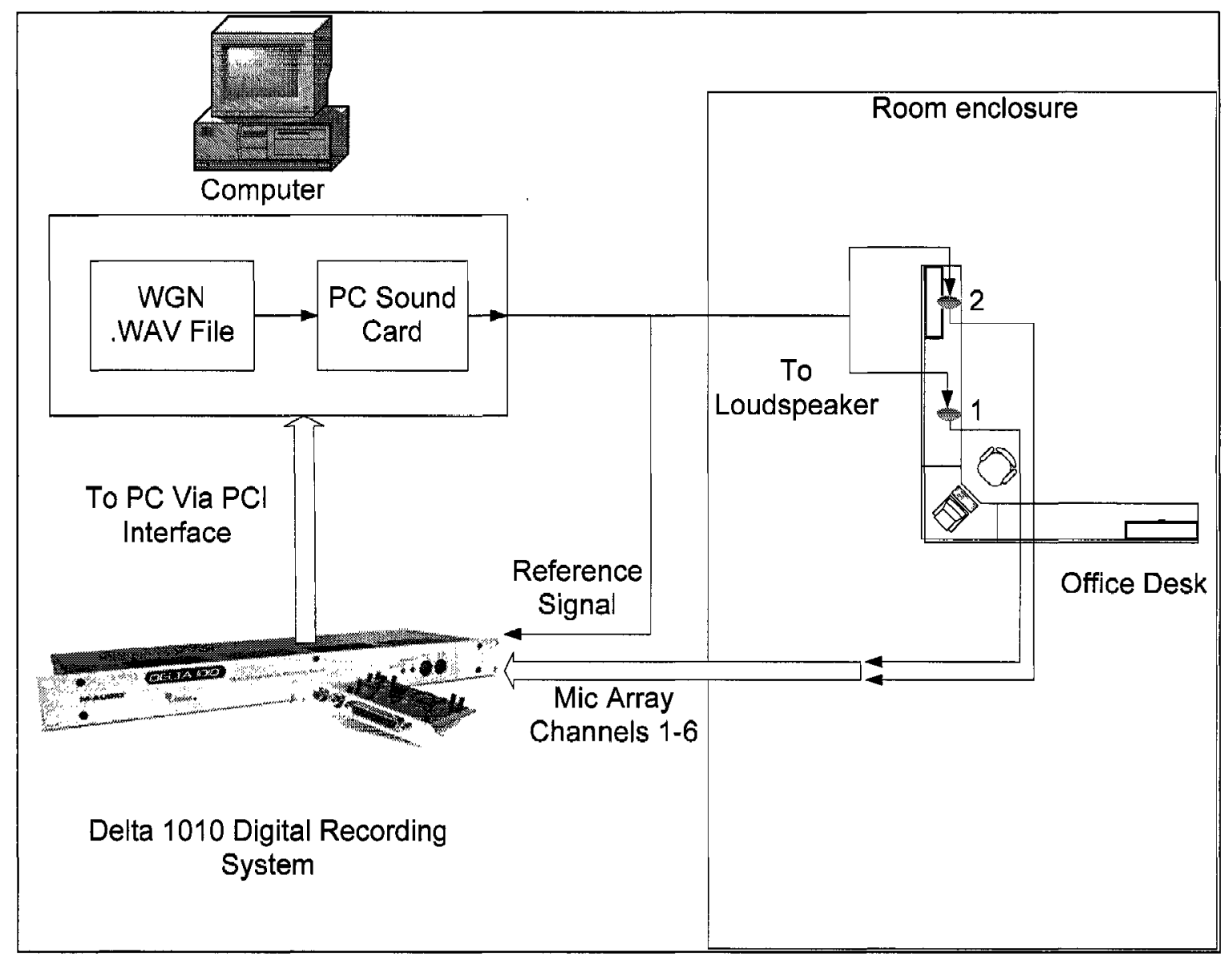

FIGURE 4.4 LRM IR Changing Room Condition Experimental Setup

The resulting acquired LRM IRs under the two different office room configurations are shown in Figure 4.5 and 4.6 respectively below. 

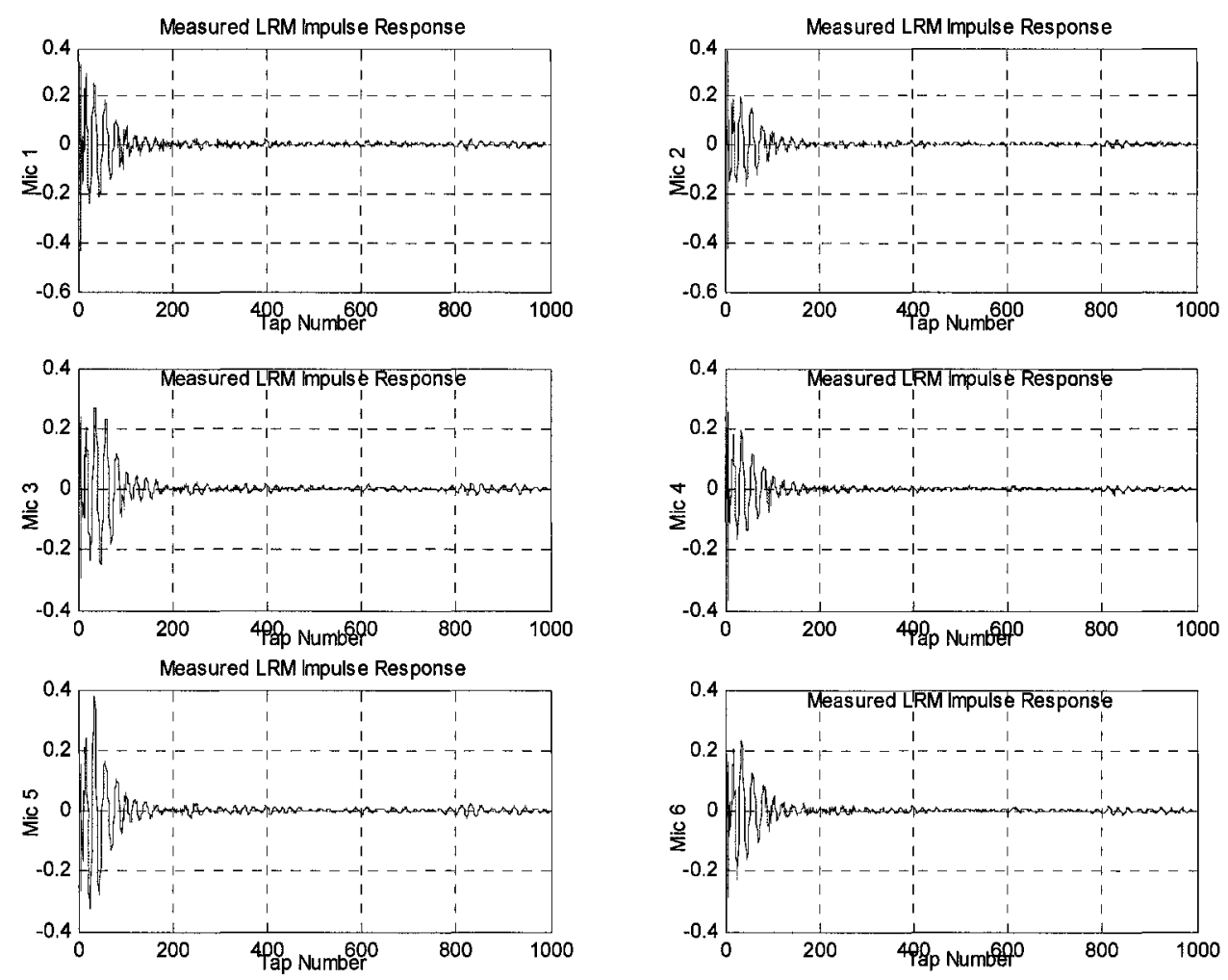

FIGURE 4.5 LRM IRs Obtained Under Room Configuration One

As shown in Figure 4.5 above, the individual LRM IRs were slightly different from one another which was expected. 

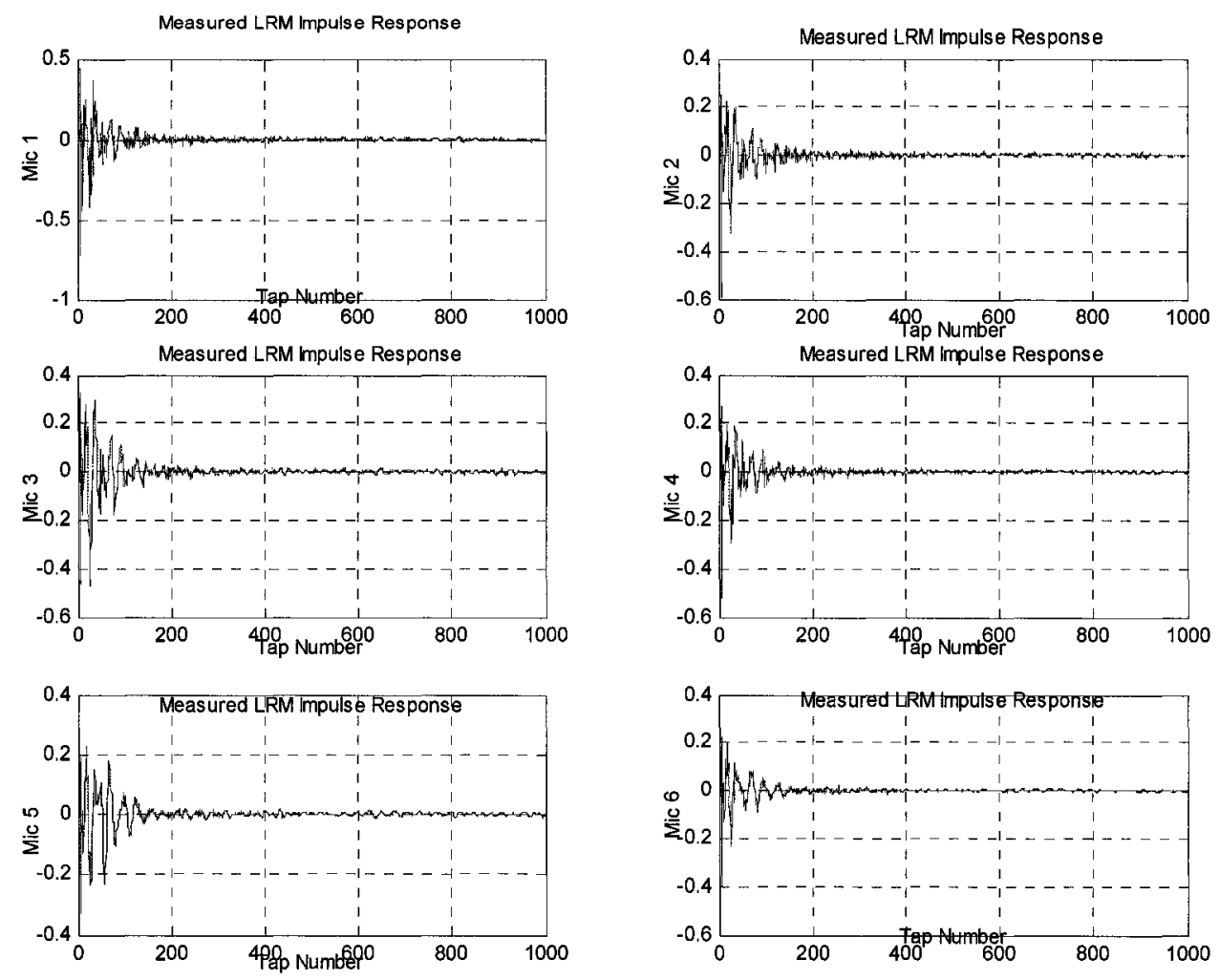

FIGURE 4.6 LRM IRs Obtained Under Room Configuration Two

The two sets of acquired LRM IRs were drastically different from one another and were used to create artificial microphone data in the changing room simulations of Chapter 6. Again, each individual LRM IR shown in Figure 4.6 was slightly different from one another as expected.

It should also be noted that based on the LRM IRs acquired under changing room environments that the first several taps of each IR remained fairly constant under the changing room conditions. This can be attributed to the physical coupling of the microphone array and speaker remaining constant under the changing room environments. It was observed that the vast majority of the changes in the LRM IRs 
occurred in approximately the next hundred taps. This was a result of the immediate surroundings changing with respect to the microphone array. The remainder of the LRM IR taps experienced relatively small changes due to the changing hands-free environment. This was due to a relatively constant echo path outside of the locally changed hands-free environment. 


\section{CHAPTER 5 SIMULATION OF STRUCTURES UNDER FIXED AND CHANGING SECTOR BASED}

BEAMFORMING CONDITIONS

\subsection{Simulation Environment and Methodology}

In order to help facilitate the simulation of the structures under investigation in this thesis a MATLAB graphical user interface (GUI) was constructed. A screen shot of the MATLAB GUI is shown in Figure 5.1 below. 


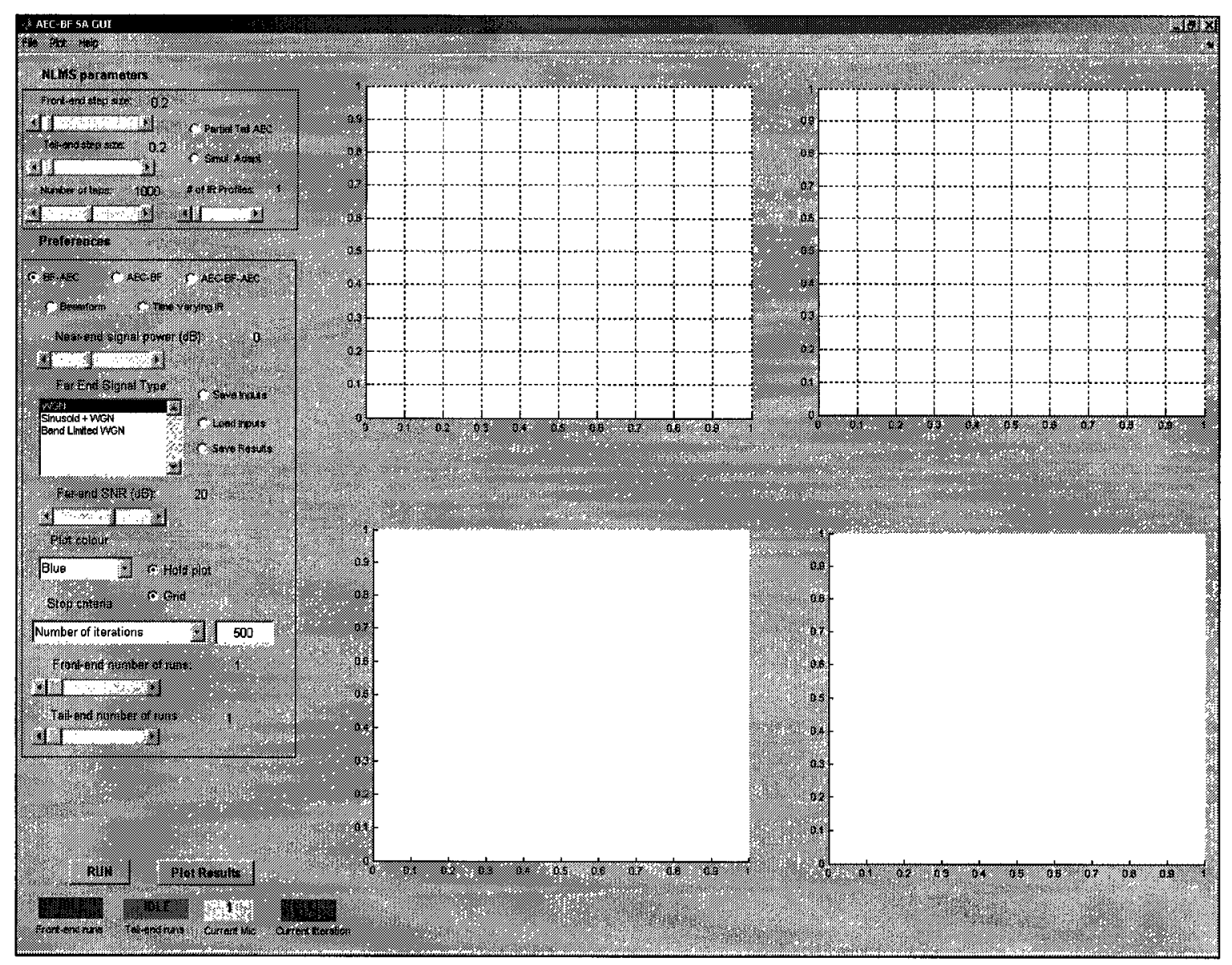

FIGURE 5.1 Blank MATLAB GUI Screen Shot

In order to run a simulation using the GUI many simulation parameters needed to be set. The typical sequence in which a simulation of one of the structures under investigation was performed is as follows:

- A set of LRM IRs were selected, via the File drop down menu, in order to create artificial microphone signals (see Equation 4)

- NLMS adaptive filter parameters, including adaptation step size and filter length, were set at the top left of the GUI under the NLMS parameters section

- The structure type to simulate along with the near-end and far-end signal characteristics were set in the left middle portion of the GUI 
- The length of and number of times to repeat the adaptation process along with optional parameters such as:

- saving input parameters to a .mat file

- saving results to a .mat file

- setting plot characteristics

were also set in the left middle portion of the GUI under the Preferences section

Once the above simulation parameters were set the Run button in the bottom left of the GUI was selected. If the structure to be simulated included a BF then a BF GUI opened as shown in Figure 5.2 below, otherwise the simulation for the single microphone structure (see Figure 3.1) was initiated. If the structure to be simulated included a BF then the following BF parameter needed to be set:

- Number of microphones in the microphone array 


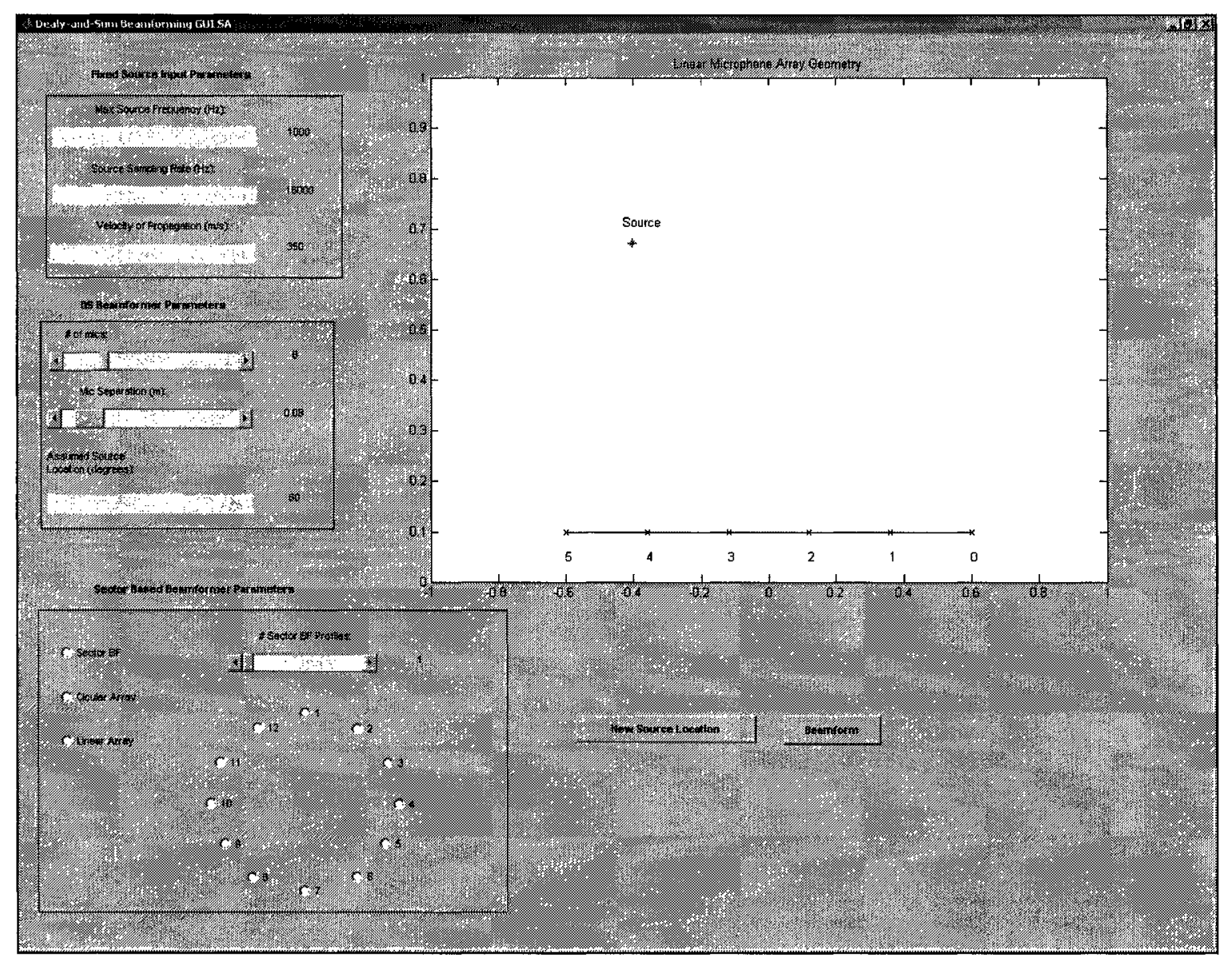

FIGURE 5.2 MATLAB BF GUI Screen Shot

If the simulation required that the $\mathrm{BF}$ change sectors then the following sector-based $\mathrm{BF}$ parameters needed to be set in the bottom left of the BF GUI:

- The Sector BF radio button needed to be selected followed by the Circular or Linear Array radio button

- If a linear array was selected then the microphone separation needed to be set such that the spatial aliasing constraint was satisfied as discussed in [53].

- The number of sector switches along with the starting sector and sectors to switch to needed to be selected 
If a linear array was selected then a six sector BF scheme was used otherwise a twelve sector circular array BF scheme was used where relative microphone delays were determined as discussed in Chapter 3 Section 3.5.2. If the BF was to remain fixed during the simulation then a circular microphone array using delay values from sector one was assumed. Once the BF parameters were set the simulation was started by selecting the Beamform button in the bottom of the BF GUI.

It should also be noted that simulation parameters can be loaded from a MATLAB .mat file into the GUI and that multiple simulation parameter mat files can be loaded into the GUI in order to perform multiple consecutive simulations. Also, multiple results from previous simulations can be plotted against one another for comparison purposes by selecting the Plot Results button in the bottom left corner of the MATLAB GUI.

After the simulation of the desired structure finished results such as mean-squared error (MSE) of the adaptive filters, echo return loss enhancement (ERLE), final converged adaptive filter taps, and the frequency response of the converged filter taps were available via the main GUI screen and GUI drop down menus.

The calculation of ERLE, which is a measure of the amount of attenuation in terms of power between an echoed signal and an echo cancelled signal, was determined by the following equation:

$$
E R L E(d B)=10 \log _{10} \frac{E\left\{d^{2}(n)\right\}}{E\left\{e^{2}(n)\right\}}
$$


Where:

$d(n)$ is the signal to be echo cancelled

$e(n)$ is the signal after echo cancellation

$E\{$.$\} is the expectation operator$

Since the expectation of the signals in Equation 12 is not generally known a moving average definition was used in place of the expectation operation as discussed in [52].

The microphone signals used in all simulations throughout this thesis were created using Equation 4 where the far-end signal, $u(n)$, and local additive noise signal, $N(n)$, were WGN sources uncorrelated to each other. The following sections present simulation results for all of the structures investigated under fixed and changing BF conditions.

\subsection{Simulation of Structures under Stationary Room and Fixed BF Conditions}

For the following stationary simulations the LRM IRs of Figure 4.3 were used to create the artificial microphone signals for a six channel circular microphone array with beamforming fixed to sector one. The adaptation step size for the single microphone structure, the BF-AEC structure, and the AEC-BF structure was set to 0.2 and the adaptation process was allowed to continue for 25000 iterations. The AECs of the aforementioned structures were set to fully adapt to 1000 tap models of the LRM transfer functions. The ERLE simulation results presented for all structures in this thesis were 
calculated between each microphone and the output of the entire system and then averaged.

\subsubsection{Stationary Room and Fixed BF Simulation Results}

\subsubsection{Simulation Results under Increasing Front-End AEC Taps}

Figure 5.3 (shown below) displays ERLE simulation results for all current structures and for the proposed structure where the size of the front-end AECs (see Figure 3.4) were increased from 40 taps up to 70 taps while the front and tail-end AEC step sizes were held constant at 0.2 . 


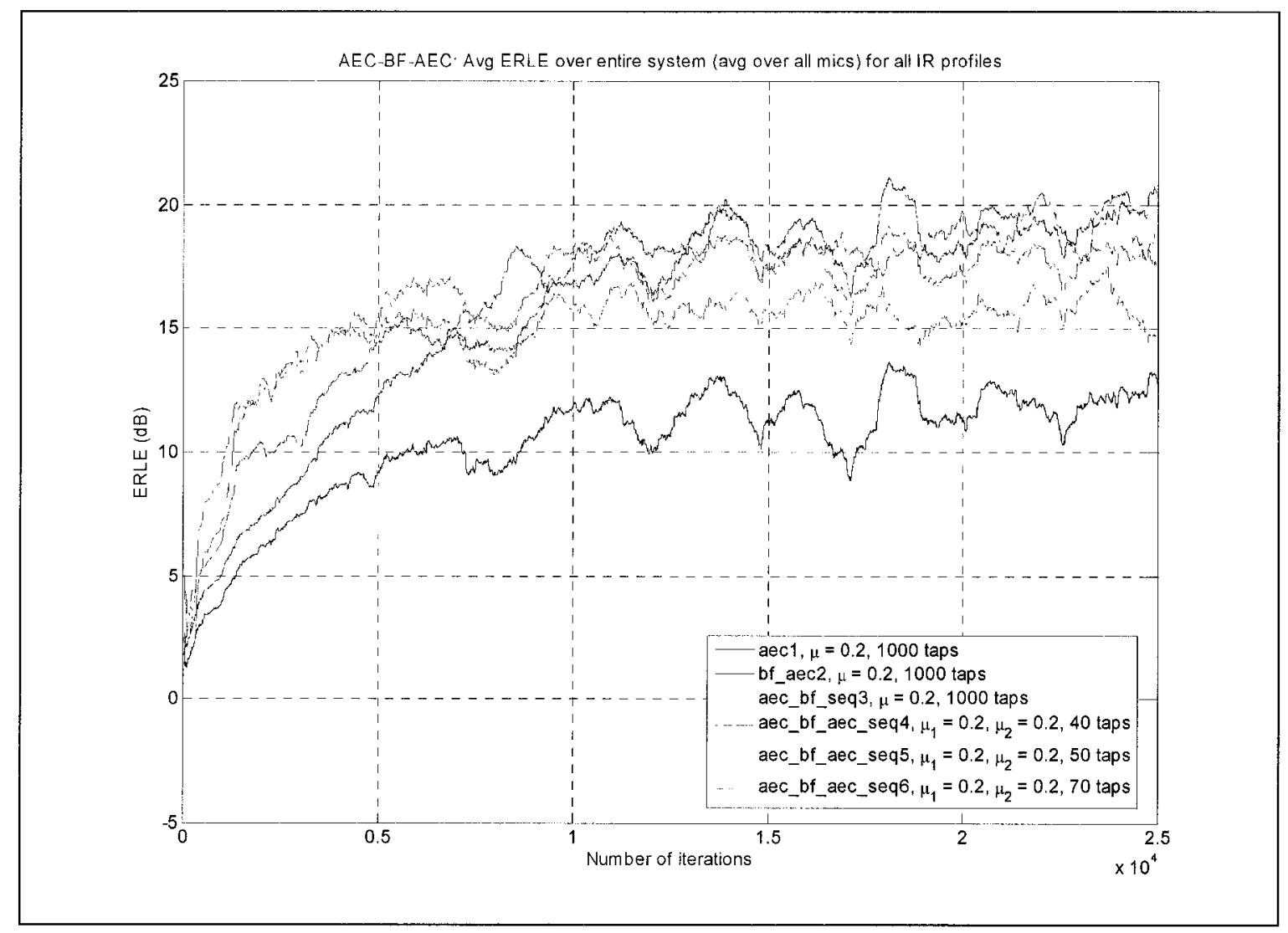

FIGURE 5.3 ERLE Results under Stationary Room Conditions with Increasing Front-End AEC Taps for Proposed Structure

As shown in Figure 5.3 the single microphone structure, indicated by the black curve, had the worst overall ERLE performance out of all the structures. The BF-AEC and AEC-BF structures attained similar steady state ERLE performance as indicated by the blue and red curves respectively. As the number of front-end AEC taps increased from 40 to 50 and finally 70 , indicated by the green, yellow, and cyan curves respectively, for the AECBF-AEC structure the overall steady state ERLE performance improved. At 70 front-end AEC taps the proposed AEC-BF-AEC structure's ERLE performance trailed the BF-AEC and $\mathrm{AEC}-\mathrm{BF}$ structures performance by no more than approximately 1 or $2 \mathrm{~dB}$ at any one instance. Also, it should be noted that the proposed AEC-BF-AEC structure displayed faster initial convergence at all front-end AEC lengths during the first 7500 iterations 
than all of the current structures. A summary of the average ERLE performance for all structures after convergence is given in Table 5.1 below, where all structures were assumed to be converged after 10000 iterations as shown in Figure 5.3.

\begin{tabular}{|c|c|c|c|c|c|c|}
\hline \multicolumn{1}{c|}{} & $\begin{array}{c}\text { AEC } \\
\text { Only }\end{array}$ & AEC & AEC- & $\begin{array}{r}\text { AEC-BF- } \\
\text { BEF 40 }\end{array}$ & $\begin{array}{c}\text { AEC-BF- } \\
\text { AEC 50 } \\
\text { Taps }\end{array}$ & $\begin{array}{c}\text { AEC-BF- } \\
\text { AEC 70 } \\
\text { Taps }\end{array}$ \\
\hline $\begin{array}{c}\text { Avg. } \\
\text { ERLE } \\
\text { (dB) }\end{array}$ & 11.69 & 18.66 & 18.63 & 15.77 & 16.66 & 17.71 \\
\hline
\end{tabular}

TABLE 5.1 Average Steady State ERLE under Stationary Room Conditions with Increasing Front-End AEC Taps for Proposed Structure

After the structures reached steady state the proposed structure's average ERLE performance using 70 front-end AEC taps lagged the BF-AEC and AEC-BF structures by less than a single $\mathrm{dB}$ and was more than $6 \mathrm{~dB}$ higher than the performance of the single microphone structure. At 40 and 50 front-end AEC taps the proposed structure's average ERLE performance trailed the BF-AEC and AEC-BF structures by about 3 and $2 \mathrm{~dB}$ respectively but was higher than the single microphone structure by approximately 4 and $5 \mathrm{~dB}$ respectively.

\subsubsection{Simulation Results under Increasing Front-End AEC Taps Towards Full Echo Path Modeling}

Figure 5.4 (see below) displays ERLE simulation results for all current structures and for the proposed structure where the size of the front-end AECs were increased from 50 taps 
towards full modeling of the individual LRM IRs while the front and tail-end AEC step sizes were held constant at 0.2 .

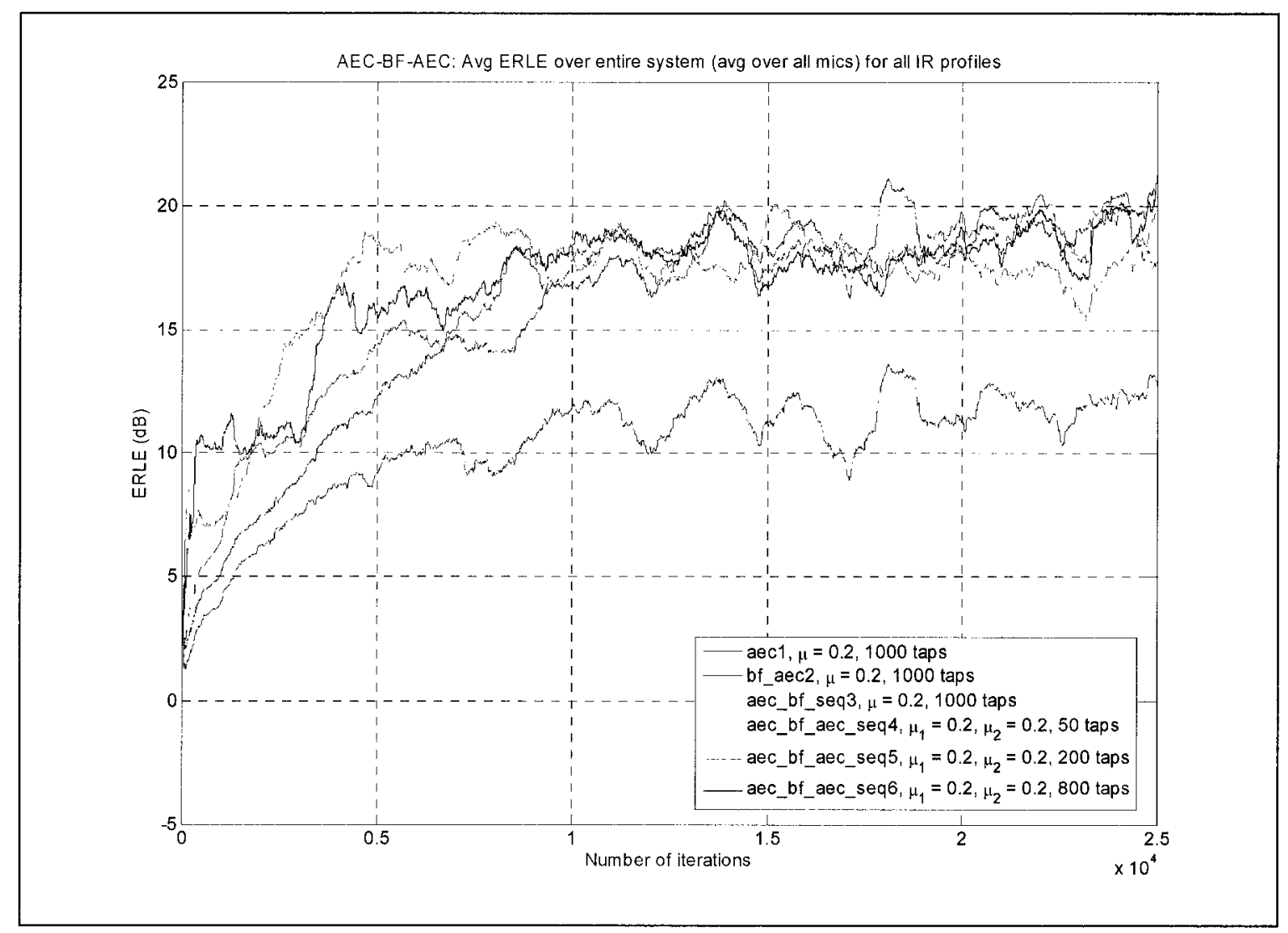

\section{FIGURE 5.4 ERLE Results under Stationary Room Conditions with Increasing Front-End AEC Taps Towards Full Echo Path Modeling for Proposed Structure}

The single microphone, BF-AEC, and AEC-BF structures ERLE performance, indicated by the purple, blue and red curves respectively, was the same as in Figure 5.3. The overall ERLE performance of the proposed AEC-BF-AEC structure improved as the number of front-end AEC taps increased from 50 to 200 and finally 800 taps, as shown by the yellow, cyan, and black curves in Figure 5.4. At 800 front-end AEC taps the proposed AEC-BF-AEC structure's performance was almost identical to the BF-AEC and AEC-BF structures but was only slightly better than the ERLE performance using 70 
front-end AEC taps as shown by the cyan curve in Figure 5.3. A summary of the average ERLE performance for all structures after convergence is given in Table 5.2 below, where all structures were again assumed to be converged after 10000 iterations as shown in Figure 5.4.

\begin{tabular}{|c|c|c|c|c|c|c|}
\cline { 2 - 6 } \multicolumn{1}{c|}{} & $\begin{array}{c}\text { AEC } \\
\text { Only }\end{array}$ & AEC & $\begin{array}{c}\text { AEC- } \\
\text { BF }\end{array}$ & $\begin{array}{c}\text { AEC-BF- } \\
\text { AEC 50 } \\
\text { Taps }\end{array}$ & $\begin{array}{r}\text { AEC-BF- } \\
\text { AEC 200 } \\
\text { Taps }\end{array}$ & $\begin{array}{c}\text { AEC-BF- } \\
\text { AEC 800 } \\
\text { Taps }\end{array}$ \\
\hline $\begin{array}{c}\text { Avg. } \\
\text { ERLE } \\
\text { (dB) }\end{array}$ & 11.69 & 18.66 & 18.63 & 16.66 & 17.77 & 18.30 \\
\hline
\end{tabular}

TABLE 5.2 Average Steady State ERLE under Stationary Room Conditions with Increasing Front-End AEC Taps Towards Full Echo Path Modeling for Proposed Structure

The average steady state ERLE performance of the proposed structure using 800 frontend AEC taps was approximately the same as the BF-AEC and AEC-BF structures with only about $0.3 \mathrm{~dB}$ difference on average. At 50 and 200 front-end AEC taps the proposed structure's average steady state ERLE performance trailed the BF-AEC and AEC-BF structures by approximately 2 and $1 \mathrm{~dB}$ respectively. Again, at all front-end AEC tap lengths from 50 to 800 the proposed structure outperformed the single microphone structure by about 5 to $6.6 \mathrm{~dB}$ respectively.

It should be noted that the gain in average steady state ERLE performance of the proposed structure by increasing the front-end AEC tap lengths from 70 to 200 and finally 800 (see Tables 5.1 and 5.2) was minimal or even slightly worse considering the substantial increase in the front-end AECs adaptive filter length. 


\subsubsection{Simulation Results under Increasing Front-End AEC Step Size}

Figure 5.5 (shown below) displays ERLE simulation results for all current structures and for the proposed structure where the front-end AECs adaptation step sizes were increased from 0.1 to 0.4 while the tail-end AEC adaptation step size was held constant at 0.2 . Also, the front-end AECs adaptive filter lengths were held constant at 80 taps.

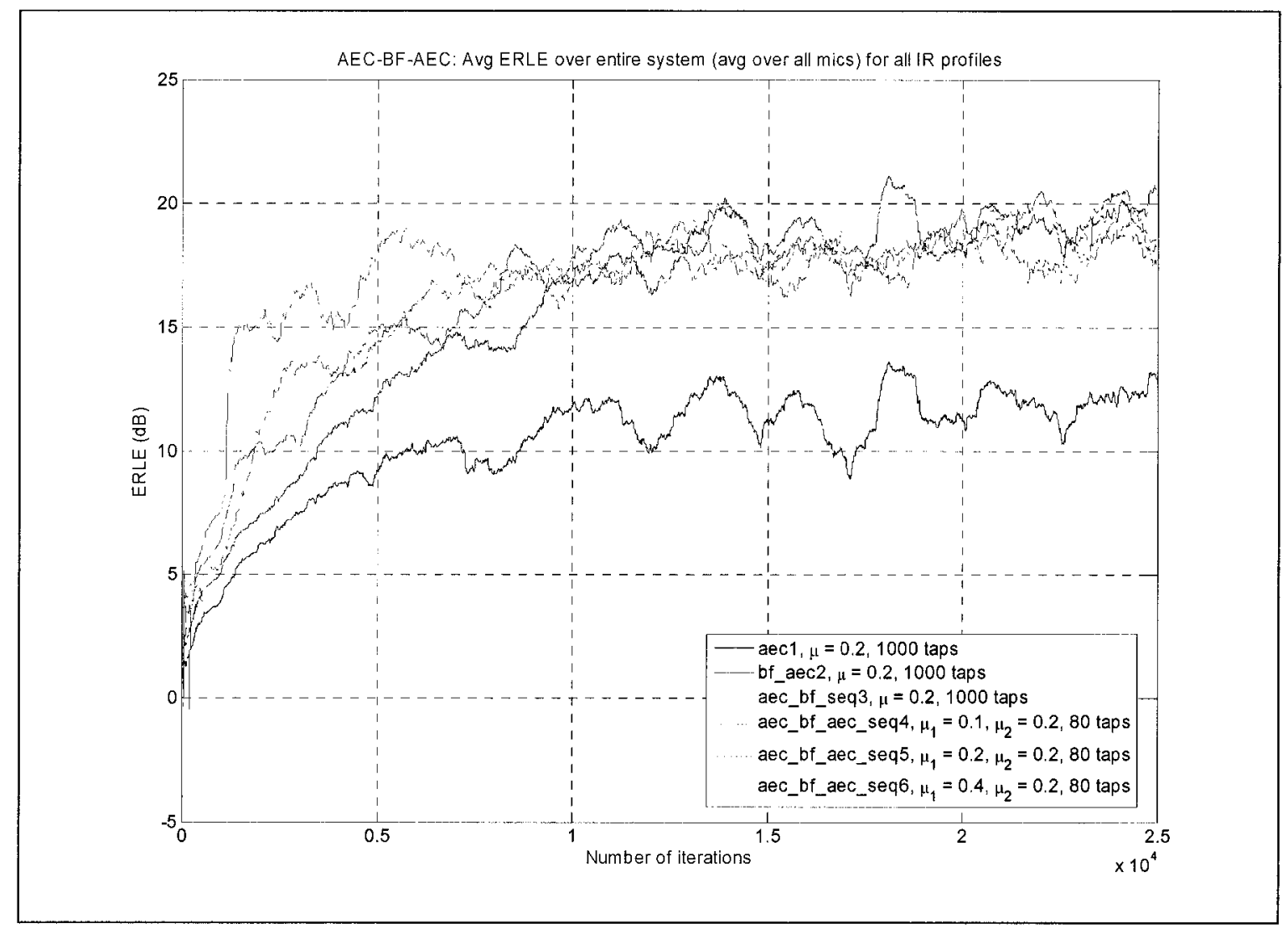

FIGURE 5.5 ERLE Results under Stationary Room Conditions with Increasing Front-End AEC Step Sizes for Proposed Structure

The single microphone, BF-AEC, and AEC-BF structures ERLE performance, indicated by the black, blue and red curves respectively, was the same as in Figure 5.3. The overall ERLE performance of the proposed AEC-BF-AEC structure degraded as the front-end 
AEC step size increased from 0.1 to 0.2 and finally 0.4 , as shown by the green, cyan, and yellow curves in Figure 5.5. As the front-end AECs step sizes, $\mu_{1}$, were increased the proposed structure converged faster than the other structures but to a lower steady state ERLE due to higher MSE caused by the larger adaptation step sizes. A summary of the average ERLE performance for all structures after convergence is given in Table 5.3 below, where all structures were again assumed to be converged after 10000 iterations as shown in Figure 5.5.

\begin{tabular}{|c|c|c|c|c|c|c|}
\cline { 2 - 6 } \multicolumn{1}{c|}{} & $\begin{array}{c}\text { AEC } \\
\text { Only }\end{array}$ & $\begin{array}{c}\text { BF- } \\
\text { AEC }\end{array}$ & $\begin{array}{c}\text { AEC- } \\
\text { BF }\end{array}$ & $\begin{array}{c}\text { AEC-BF- } \\
\text { AEC } \boldsymbol{\mu}_{1}=\mathbf{0 . 1}\end{array}$ & $\begin{array}{c}\text { AEC-BF- } \\
\text { AEC } \boldsymbol{\mu}_{\mathbf{1}}=\mathbf{0 . 2}\end{array}$ & $\begin{array}{c}\text { AEC-BF- } \\
\text { AEC } \boldsymbol{\mu}_{\mathbf{1}}=\mathbf{0 . 4}\end{array}$ \\
\hline $\begin{array}{c}\text { Avg. } \\
\text { ERLE } \\
\text { (dB) }\end{array}$ & 11.69 & 18.66 & 18.63 & 18.18 & 17.73 & 16.14 \\
\hline
\end{tabular}

TABLE 5.3 Average Steady State ERLE under Stationary Room Conditions with Increasing Front-End AEC Step Sizes for Proposed Structure

The average steady state ERLE performance of the proposed structure using a front-end step size of 0.1 was approximately the same as the BF-AEC and AEC-BF structures with only about $0.5 \mathrm{~dB}$ difference on average. At front-end AEC step sizes of 0.2 and 0.4 the proposed structure's average steady state ERLE performance trailed the BF-AEC and AEC-BF structures by approximately 1 and $2.5 \mathrm{~dB}$ respectively. Also, at all front-end AEC step sizes from 0.1 to 0.4 the proposed structure outperformed the single microphone structure by about 6.5 to $4.5 \mathrm{~dB}$ respectively. 


\subsubsection{Simulation Results under Increasing Tail-End AEC Step Size}

Figure 5.6 (shown below) displays ERLE simulation results for all current structures and for the proposed structure where the tail-end AEC step size was increased from 0.1 to 0.4 while the front-end AECs step sizes were held constant at 0.2. Also, the front-end AECs adaptive filter lengths were held constant at 80 taps.

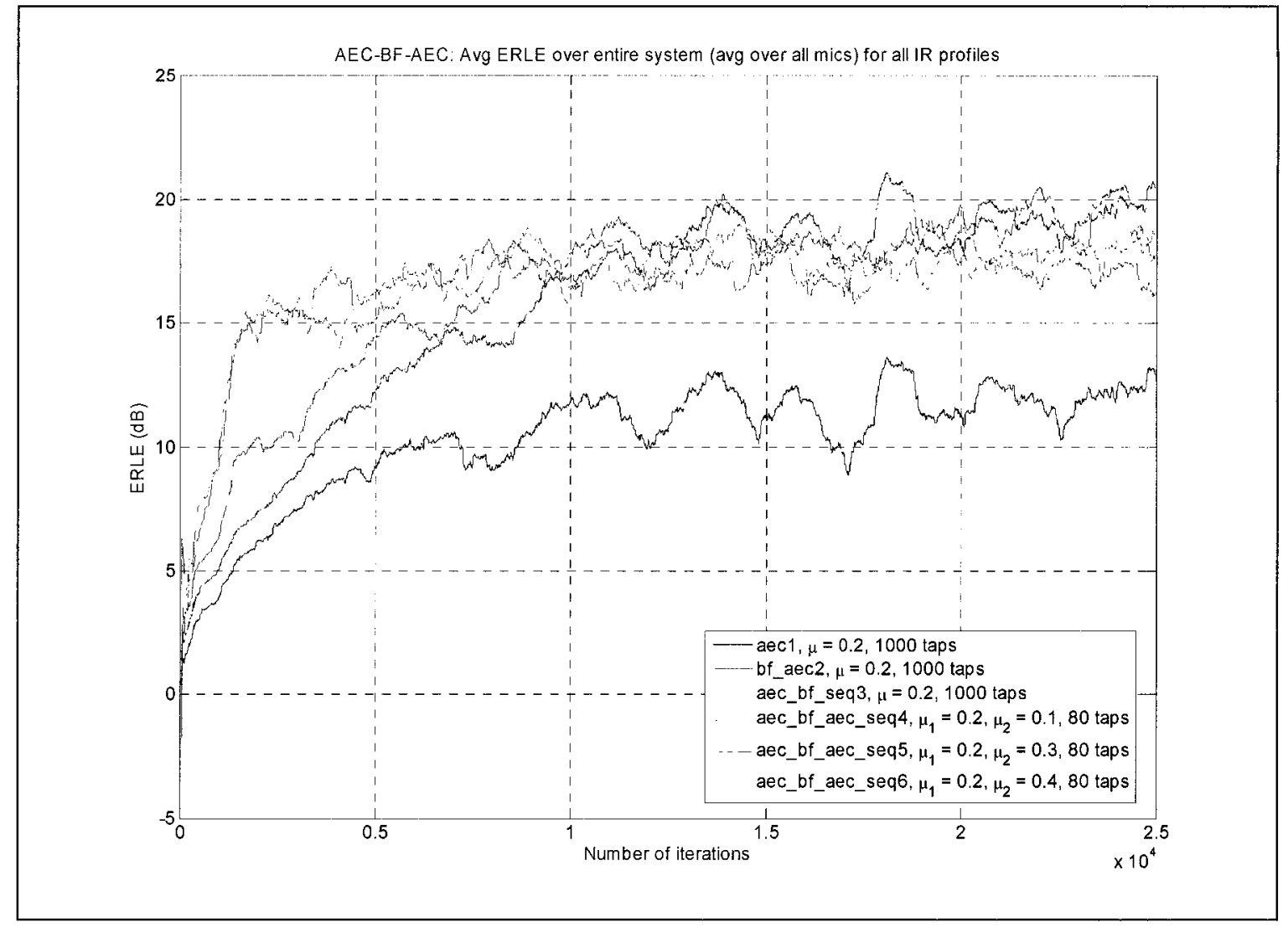

FIGURE 5.6 ERLE Results under Stationary Room Conditions with Increasing Tail-End AEC Step Sizes for Proposed Structure

The single microphone, BF-AEC, and AEC-BF structures ERLE performance, indicated by the black, blue and red curves respectively, was the same as in Figure 5.3. The overall ERLE performance of the proposed AEC-BF-AEC structure degraded as the tail-end AEC step size increased from 0.1 to 0.3 and finally 0.4 , as shown by the green, purple, 
and yellow curves in Figure 5.6. As the tail-end AEC step size, $\mu_{2}$, was increased the proposed structure converged faster than the other structures but to a lower steady state ERLE due to higher MSE caused by the larger adaptation step sizes. A summary of the average ERLE performance for all structures after convergence is given in Table 5.4 below, where all structures were again assumed to be converged after 10000 iterations as shown in Figure 5.6.

\begin{tabular}{|c|c|c|c|c|c|c|}
\cline { 2 - 6 } \multicolumn{1}{c|}{} & $\begin{array}{c}\text { AEC } \\
\text { Only }\end{array}$ & $\begin{array}{c}\text { BF- } \\
\text { AEC }\end{array}$ & $\begin{array}{c}\text { AEC- } \\
\text { BF }\end{array}$ & $\begin{array}{c}\text { AEC-BF- } \\
\text { AEC } \boldsymbol{\mu}_{2}=0.1\end{array}$ & $\begin{array}{c}\text { AEC-BF- } \\
\text { AEC } \mu_{2}=0.3\end{array}$ & $\begin{array}{c}\text { AEC-BF- } \\
\text { AEC } \boldsymbol{\mu}_{2}=\mathbf{0 . 4}\end{array}$ \\
\hline $\begin{array}{c}\text { Avg. } \\
\text { ERLE } \\
\text { (dB) }\end{array}$ & 11.69 & 18.66 & 18.63 & 17.59 & 17.41 & 17.00 \\
\hline
\end{tabular}

TABLE 5.4 Average Steady State ERLE under Stationary Room Conditions with Increasing Tail-End AEC Step Sizes for Proposed Structure

The average steady state ERLE performance of the proposed structure using a tail-end step size of 0.1 was approximately $1 \mathrm{~dB}$ less on average than the BF-AEC and AEC-BF structures. At tail-end AEC step sizes of 0.3 and 0.4 the proposed structure's average steady state ERLE performance trailed the BF-AEC and AEC-BF structures by approximately 1.2 and $1.6 \mathrm{~dB}$ respectively. Also, at all tail-end AEC step sizes ranging from 0.1 to 0.4 the proposed structure outperformed the single microphone structure by about 5.9 to $5.3 \mathrm{~dB}$ respectively.

Also, it should be noted that the impact on average steady state ERLE performance for the proposed structure was greater when the front-end AECs step sizes were increased compared to increasing the tail-end AEC step sizes. As the front-end AECs step sizes 
were increased from 0.1 to 0.4 the average steady state ERLE for the proposed structure dropped by approximately $2 \mathrm{~dB}$ (see Table 5.3 ), whereas the average steady state ERLE dropped by only $0.6 \mathrm{~dB}$ when the tail-end AEC step sizes were increased (see Table 5.4).

\subsubsection{Stationary Room and Fixed BF Simulation Results Summary}

Taking the above simulation results into consideration, a summary of the trends observed on the ERLE performance of the proposed structure compared to current structures under stationary room conditions is as follows:

- As the length of front-end AEC adaptive filters was increased the ERLE performance of the proposed structure improved towards that of the BF-AEC and AEC-BF structures and outperformed the single microphone structure by at least $4 \mathrm{~dB}$ (see Figures 5.3 - 5.4 and Table 5.1)

- At 70 font-end AEC adaptive filters taps the ERLE performance of the proposed structure was very similar to that of the BF-AEC and AEC-BF structures (see Figures $5.3-5.4$ and Tables $5.1-5.2)$

- Increasing beyond 70 front-end taps resulted in a marginal increase in or slightly worse ERLE performance of the proposed structure at the expense of increased structure complexity (see Figures 5.3 - 5.4 and Tables $5.1-5.2$ )

- As the front-end and tail-end AEC adaptive filters step sizes were increased the ERLE performance of the proposed structure degraded away from the performance of the BF-AEC and AEC-BF structures but still outperformed the single microphone structure by at least $4.5 \mathrm{~dB}$ (see Figures 5.5 - 5.6 and Table 5.3) 
- Increasing the front-end AEC adaptive filters step sizes for the proposed structure had a greater negative impact on ERLE performance than increasing the tail-end AEC adaptive filter step sizes (see Figures 5.5 - 5.6 and Tables $5.3-5.4$ )

- The single microphone AEC structure had the worst overall ERLE performance of all the structures

Simulations of the proposed structure were also performed for increasing front-end and tail-end AEC adaptive filter step sizes towards and beyond 2.0. At step sizes of 2.0 and greater the proposed structure's AEC adaptive filters became divergent as discussed in Section 3.5.1.

\subsection{Simulation of Structures under Changing Sector Based BF Conditions}

For the following changing sector based BF simulations the LRM IRs or Figure 4.6 were used to create the artificial microphone signals for a six channel linear microphone array with the BF switching from sector one to sector two. The adaptation step size for the single microphone structure, the BF-AEC structure, and the AEC-BF structure was set to 0.3 and the AEC adaptive filters of the aforementioned structures were set to fully adapt to 1000 tap models of the LRM transfer functions. The adaptation process was allowed to continue for 25000 iterations with the BF fixed to sector one. Then starting at the next iteration the $\mathrm{BF}$ was switched to sector two where a new set of $\mathrm{BF}$ delay values were applied and the structures were allowed to readapt for another 25000 iterations. 
The following simulations demonstrated how the current and proposed structures were impacted when the sector based BF switched from one sector to a different active sector. This situation would have arisen when the talker in the current sector became quiet and another talker in a different sector became active. Again, it should be noted that acoustic echo cancellation was only performed when the local participants in the hands-free communication system were inactive, as discussed in Section 3.5.

\subsubsection{Changing Sector Based BF Simulation Results}

The following changing sector based BF simulations were performed using the measured LRM IRs of Figure 4.6 that were discussed in Section 4.4.2. The linear sector based BF was switched from sector one to two in order to observe the behavior of all structures under non-stationary BF operation. ERLE simulation results under these changing BF conditions are discussed below.

\subsubsection{Simulation Results under Increasing Front-End AEC Taps}

Figure 5.7 (shown below) displays ERLE simulation results for all current structures and for the proposed structure where the size of the front-end AECs (see Figure 3.4) were increased from 60 taps up to 100 taps while the front and tail-end AEC step sizes were held constant at 0.15 and 0.3 respectively. 


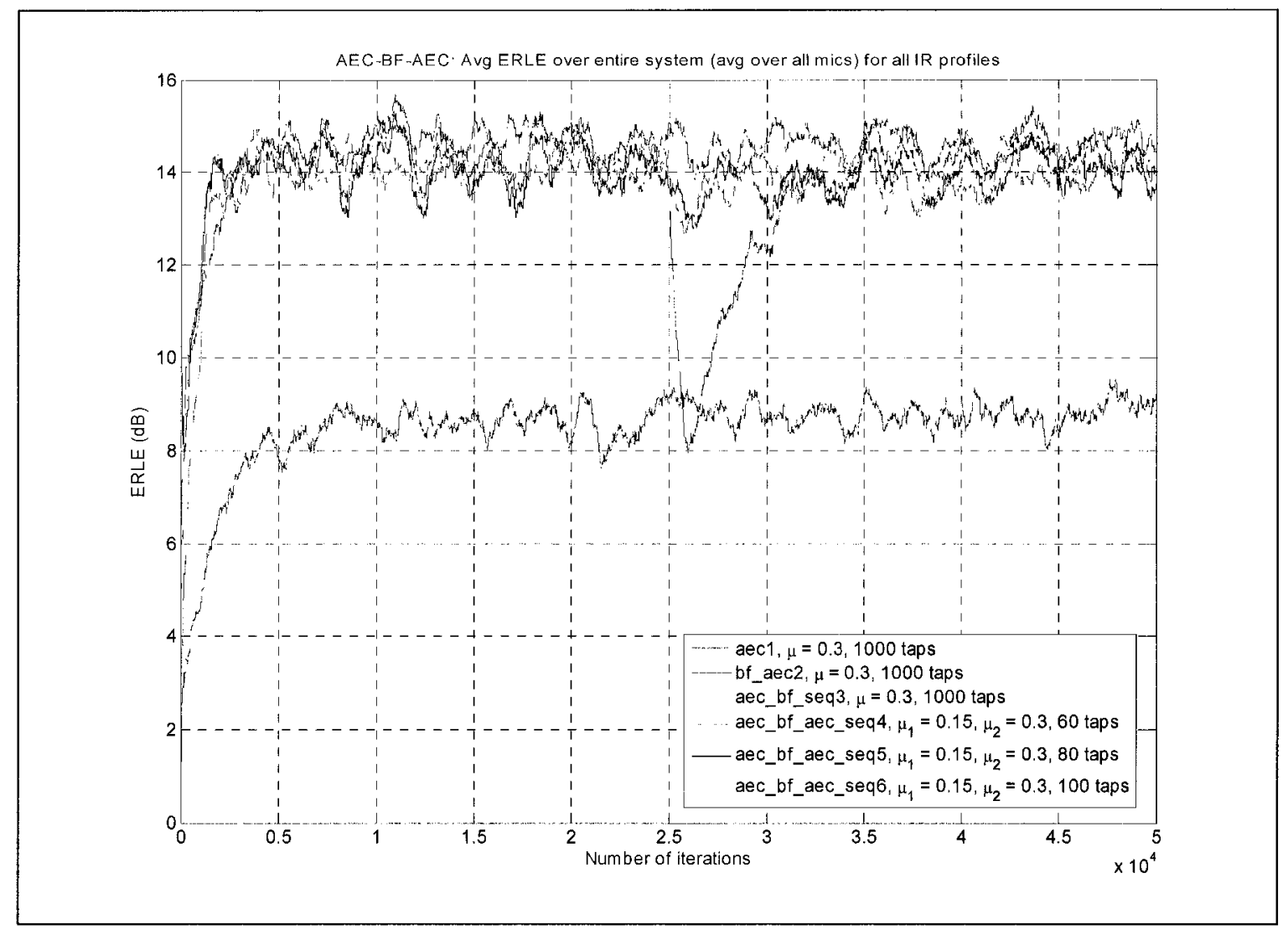

\section{FIGURE 5.7 ERLE Results under Changing Sector Based BF Conditions with Increasing} Front-End AEC Taps for Proposed Structure

As shown in Figure 5.7 the single microphone AEC only structure, indicated by the purple curve, had the worst overall ERLE performance out of all the structures and was unaffected by the BF changes. The BF-AEC structure's ERLE performance, indicated by the blue curve, suffered substantially when the BF switched sectors. This was due to the BF-AEC structure's tail-end AEC adjusting its model of the LRM IR echo path to incorporate the BF changes. Since the BF-AEC structure's AEC models the full echo path it was slow to recover from the fluctuations caused by the BF sector switch, as shown in Figure 5.7. The AEC-BF structure's ERLE performance, indicated by the red curve, was unaffected by the BF sector switch because the front-end AECs did not need to track the BF time variations. The initial steady state ERLE performance of the BF- 
AEC and AEC-BF structure before the BF sector switch and final steady state ERLE performance after the sector switch were very similar, as shown in Figure 5.7.

The ERLE performance of the proposed AEC-BF-AEC structure dropped less after the $\mathrm{BF}$ sector switch as the number of front-end AEC taps increased and significantly outperformed the BF-AEC structure during re-convergence at all front-end AEC tap lengths. This was due to the increasingly shorter number of tail-end AEC taps, as a result of the number of front-end AEC taps increasing, being able to quickly track the changes in the LRM IRs caused by the BF sector switch. Thus, re-convergence to steady state ERLE occurred faster. Also, as the number of front-end AEC taps of the proposed structure were increased from 60 to 80 and finally to 100 , indicated by the green, black, and yellow curves, the steady state ERLE performance before and after the sector change improved slightly and was similar to the steady state performance of the BF-AEC and AEC-BF structures. A summary of the average ERLE performance for all structures during initial steady state conditions, during re-convergence after the BF sector change, and during final steady state conditions is given in Table 5.5 below. All structures were assumed to be in initial steady state, re-convergence, and final steady state conditions between $7500-25000,25000-37500$, and $37500-50000$ iterations respectively as seen in Figure 5.7. 


\begin{tabular}{|c|c|c|c|c|c|c|}
\hline & $\begin{array}{l}\text { AEC } \\
\text { Only }\end{array}$ & $\begin{array}{l}\mathbf{B F}- \\
\mathbf{A E C}\end{array}$ & $\mathrm{AEC}$ & $\begin{array}{c}\text { AEC-BF- } \\
\text { AEC 60 } \\
\text { Taps } \\
\end{array}$ & $\begin{array}{c}\mathrm{AEC-BF} \\
\text { AEC 80 } \\
\text { Taps }\end{array}$ & $\begin{array}{c}\text { AEC-BF- } \\
\text { AEC 100 } \\
\text { Taps }\end{array}$ \\
\hline $\begin{array}{l}\text { Avg. ERLE during } \\
\text { initial steady state } \\
\text { (dB) }\end{array}$ & 8.74 & 14.50 & 14.55 & 14.09 & 14.09 & 14.14 \\
\hline $\begin{array}{l}\text { Avg. ERLE during } \\
\text { re-convergence (dB) }\end{array}$ & 8.74 & 12.67 & 14.55 & 13.83 & 13.79 & 14.01 \\
\hline $\begin{array}{l}\text { Avg. ERLE during } \\
\text { finial steady state } \\
\text { (dB) }\end{array}$ & 8.74 & 14.47 & 14.55 & 13.86 & 13.97 & 14.00 \\
\hline
\end{tabular}

TABLE 5.5 Average ERLE Performance under Changing Sector Based BF Conditions with Increasing Front-End AEC Taps for Proposed Structure

During initial and final steady state operation the proposed structure's average ERLE performance lagged the BF-AEC and AEC-BF structures by at most $0.69 \mathrm{~dB}$ for both cases. The single microphone structure was outperformed by the proposed structure by at least $5.12 \mathrm{~dB}$ during both initial and final steady state operation.

During re-convergence the proposed structure's average ERLE performance lagged the AEC-BF structure's by at most $0.76 \mathrm{~dB}$ and lead the BF-AEC structure's by at least $1.12 \mathrm{~dB}$ on average, as shown in Table 5.5. Again, the single microphone structure was outperformed by the proposed structure by at least $5.05 \mathrm{~dB}$ during re-convergence.

\subsubsection{Simulation Results under Increasing Front-End AEC Taps Towards Full Echo Path Modeling}

Figure 5.8 (see below) displays ERLE simulation results for all current structures and for the proposed structure where the size of the front-end AECs were increased from 60 taps 
towards full modeling of the individual LRM IRs while the front and tail-end AEC step sizes were held constant at 0.15 and 0.3 respectively.

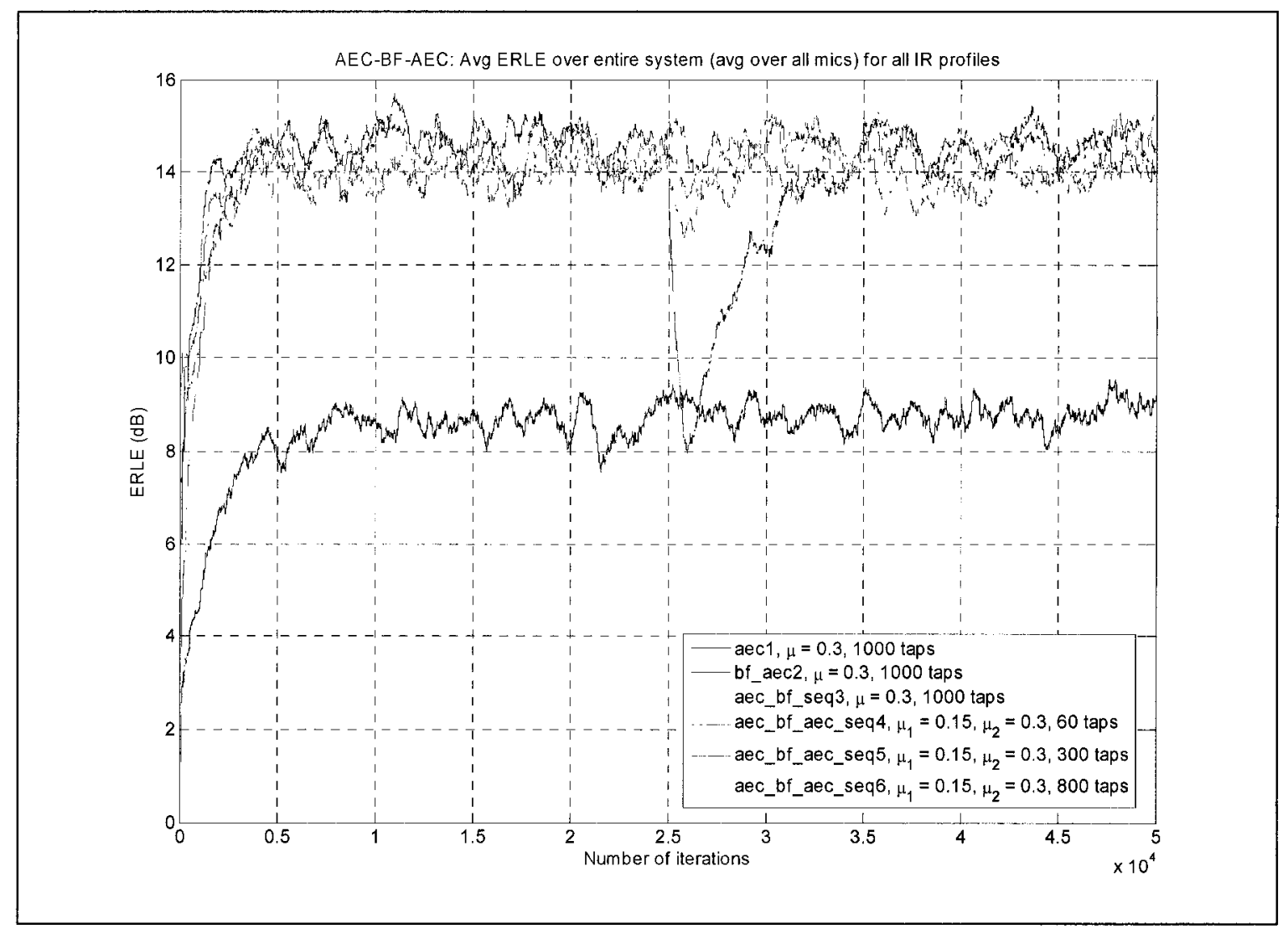

\section{FIGURE 5.8 ERLE Results under Changing Sector Based BF Conditions with Increasing Front-End AEC Taps Towards Full Echo Path Modeling for Proposed Structure}

The single microphone, BF-AEC, and AEC-BF structures ERLE performance, indicated by the black, blue and red curves respectively, was the same as in Figure 5.7. Increasing the number of front-end AEC taps of the proposed structure from 60 , to 300 , and finally to 800 caused the ERLE performance to improve towards the AEC-BF structure as shown by the green, cyan, and yellow curves respectively. As the number of front-end AEC taps increased for the proposed structure it suffered almost no repercussions when 
the BF sector switch occurred. This was a result of the increasingly smaller number of tail-end AEC taps, due to the increasingly larger number of front-end AEC taps, being able to very quickly adapt to the changes caused by the BF time variations. A summary of the average ERLE performance for all structures during initial steady state conditions, during re-convergence after the $\mathrm{BF}$ sector change, and during final steady state conditions is given in Table 5.6 below.

\begin{tabular}{|c|c|c|c|c|c|c|}
\cline { 2 - 6 } \multicolumn{1}{c|}{} & $\begin{array}{c}\text { AEC } \\
\text { Only }\end{array}$ & $\begin{array}{c}\text { BF- } \\
\text { AEC }\end{array}$ & $\begin{array}{r}\text { AEC- } \\
\text { BF }\end{array}$ & $\begin{array}{c}\text { AEC-BF- } \\
\text { AEC 60 } \\
\text { Taps }\end{array}$ & $\begin{array}{c}\text { AEC-BF- } \\
\text { AEC 300 } \\
\text { Taps }\end{array}$ & $\begin{array}{c}\text { AEC-BF- } \\
\text { AEC 800 } \\
\text { Taps }\end{array}$ \\
\hline $\begin{array}{c}\text { Avg. ERLE during } \\
\text { initial steady state } \\
\text { (dB) }\end{array}$ & 8.74 & 14.50 & 14.55 & 14.09 & 14.11 & 14.06 \\
\hline $\begin{array}{c}\text { Avg. ERLE during } \\
\text { re-convergence (dB) }\end{array}$ & 8.74 & 12.67 & 14.55 & 13.83 & 14.31 & 14.26 \\
\hline $\begin{array}{c}\text { Avg. ERLE during } \\
\text { finial steady state } \\
\text { (dB) }\end{array}$ & 8.74 & 14.47 & 14.55 & 13.86 & 14.20 & 14.18 \\
\hline
\end{tabular}

TABLE 5.6 Average ERLE Performance under Changing Sector Based BF Conditions with Increasing Front-End AEC Taps Towards Full Echo Path Modeling for Proposed Structure

Increasing the number of front-end AEC taps for the proposed structure to 300 and 800 resulted in only marginal increases in initial and final steady state ERLE performance compared to 100 taps (see Table 5.5). Even during re-convergence the average ERLE performance of the proposed structure at 300 and 800 front-end AEC taps was only at most $0.3 \mathrm{~dB}$ greater than at 100 taps (See Table 5.5). 


\subsubsection{Simulation Results under Increasing Front-End AEC Step Size}

Figure 5.9 (shown below) displays ERLE simulation results for all current structures and for the proposed structure where the front-end AECs adaptation step sizes were increased from 0.1 to 0.3 while the tail-end AEC adaptation step size was held constant at 0.3 . Also, the front-end AECs adaptive filter lengths were held constant at 80 taps.

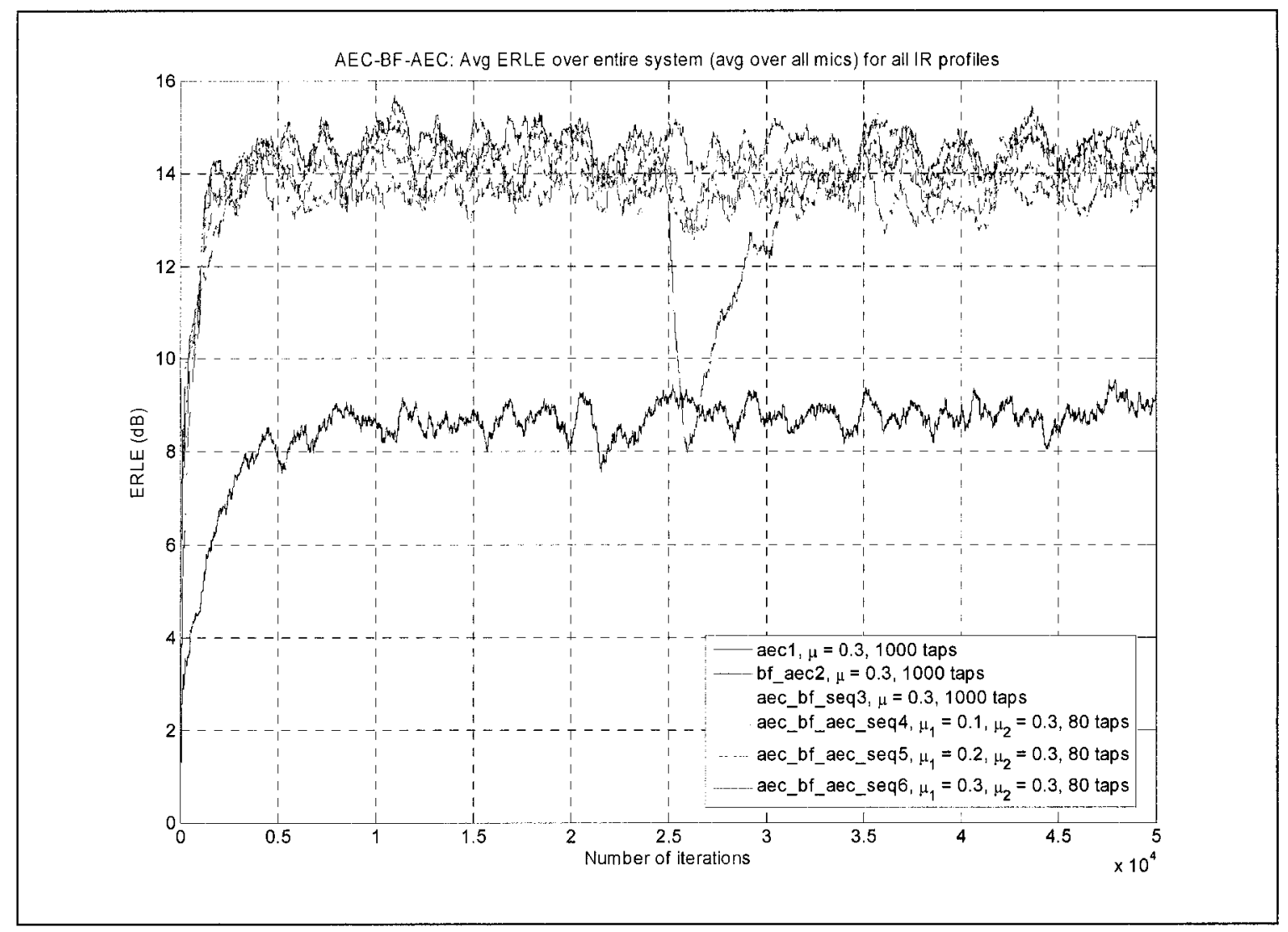

\section{FIGURE 5.9 ERLE Results under Changing Sector Based BF Conditions with Increasing Front-End AEC Step Sizes for Proposed Structure}

The single microphone, BF-AEC, and AEC-BF structures ERLE performance, indicated by the black, blue and red curves respectively, was the same as in Figure 5.7. The initial and final steady state performance of the proposed AEC-BF-AEC structure degraded away from that of the BF-AEC and AEC-BF structures as the front-end AECs step sizes 
increased, as indicated by the green, cyan, and purple curves in Figure 5.9. The lower steady state ERLE performance was a result of the higher MSE caused by the increasing front-end AEC step sizes. After the BF sector switch the proposed structure attained slightly higher ERLE performance during re-convergence at smaller front-end AEC step sizes due to the correspondingly lower MSE. Increasing the front-end AECs step sizes did not help the proposed structure re-converge faster after the BF sector switch because the front-end AECs do not feel the effects of the sector switch. A summary of the average ERLE performance for all structures during initial steady state conditions, during re-convergence after the $\mathrm{BF}$ sector change, and during final steady state conditions is given in Table 5.7 below.

\begin{tabular}{|c|c|c|c|c|c|c|}
\hline & $\begin{array}{l}\text { AEC } \\
\text { Only }\end{array}$ & BE- & $\begin{array}{c}\mathrm{AEC}- \\
\mathrm{BF}\end{array}$ & $\begin{array}{c}\mathrm{AEC-BF}- \\
\mathrm{AEC} \\
\mu_{1}=0.1\end{array}$ & $\begin{array}{c}\mathrm{AEC}-\mathrm{BF}- \\
\mathrm{AEC} \\
\mu_{1}=0.2\end{array}$ & $\begin{array}{c}\text { AEC-BF- } \\
\text { AEC } \\
\mu_{1}=0.3\end{array}$ \\
\hline $\begin{array}{l}\text { Avg. ERLE during } \\
\text { initial steady state } \\
\text { (dB) }\end{array}$ & 8.74 & 14.50 & 14.55 & 14.25 & 13.98 & 13.67 \\
\hline $\begin{array}{l}\text { Avg. ERLE during } \\
\text { re-convergence (dB) }\end{array}$ & 8.74 & 12.67 & 14.55 & 13.95 & 13.78 & 13.55 \\
\hline $\begin{array}{l}\text { Avg. ERLE during } \\
\text { finial steady state } \\
\text { (dB) }\end{array}$ & 8.74 & 14.47 & 14.55 & 14.14 & 13.80 & 13.50 \\
\hline
\end{tabular}

TABLE 5.7 Average ERLE Performance under Changing Sector Based BF Conditions with Increasing Front-End AEC Step Sizes for Proposed Structure

During initial and final steady state operation the proposed structure's average ERLE performance lagged the BF-AEC and AEC-BF structures by at most $1.05 \mathrm{~dB}$ for both cases. The single microphone structure was outperformed by the proposed structure by at least $4.76 \mathrm{~dB}$ during both initial and final steady state operation. 
During re-convergence the proposed structure's average ERLE performance lagged the AEC-BF structure's by at most $1.0 \mathrm{~dB}$ and lead the BF-AEC structure's by at least $0.88 \mathrm{~dB}$ on average, as shown in Table 5.7. Again, the single microphone structure was outperformed by the proposed structure by at least $4.81 \mathrm{~dB}$ during re-convergence.

\subsubsection{Simulation Results under Increasing Tail-End AEC Step Size}

Figure 5.10 (shown below) displays ERLE simulation results for all current structures and for the proposed structure where the tail-end AEC adaptation step size was increased from 0.1 to 0.3 while the front-end AECs adaptation step sizes were held constant at 0.15 . Also, the front-end AECs adaptive filter lengths were held constant at 60 taps. 


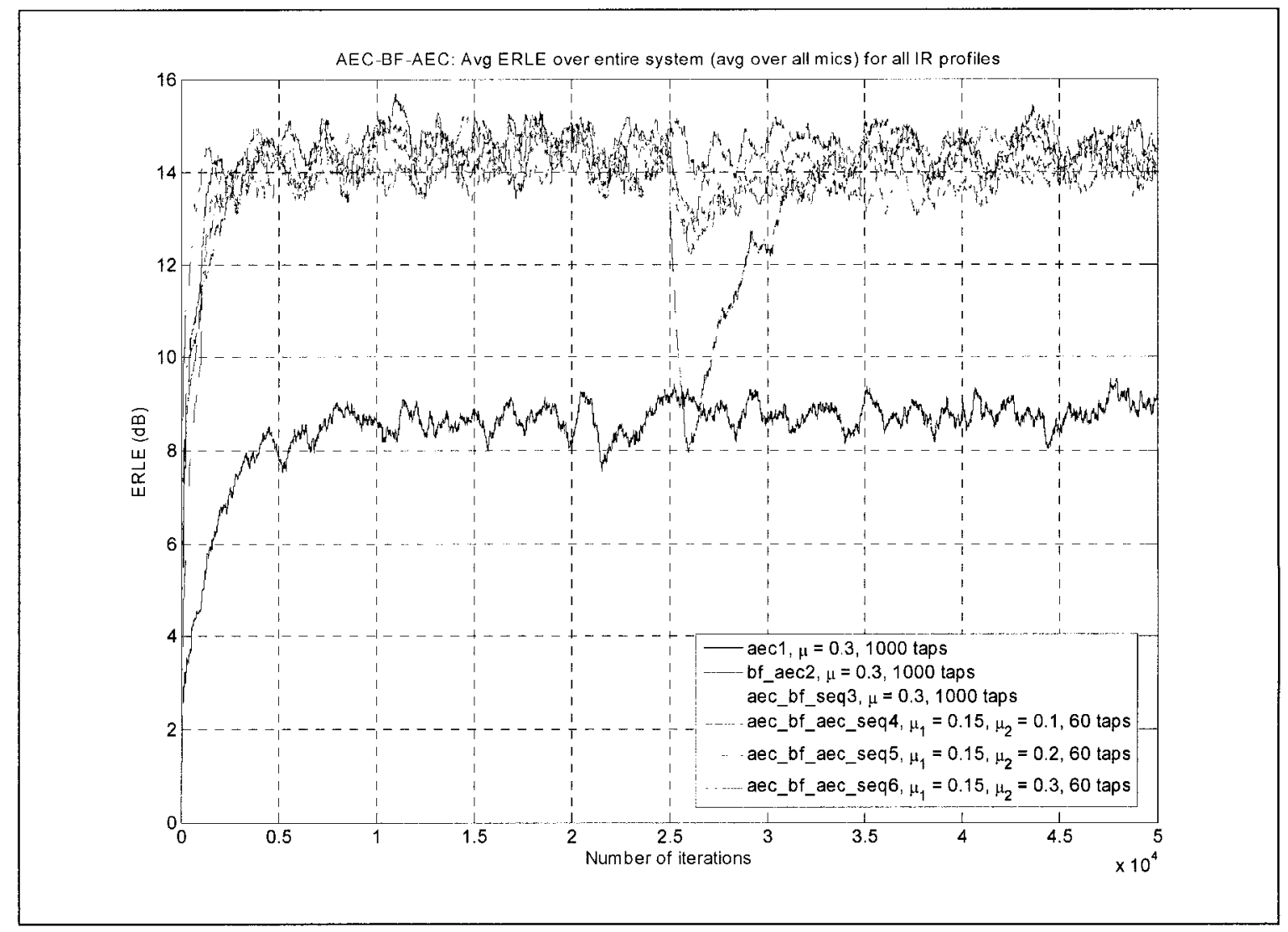

\section{FIGURE 5.10 ERLE Results under Changing Sector Based BF Conditions with Increasing} Tail-End AEC Step Sizes for Proposed Structure

The single microphone, BF-AEC, and AEC-BF structures ERLE performance, indicated by the black, blue and red curves respectively, was the same as in Figure 5.7. The initial and final steady state performance of the proposed structure degraded away from that of the BF-AEC and AEC-BF structures as the tail-end AEC step sizes increased, as indicated by the cyan, purple, and green curves in Figure 5.10. This lower ERLE performance for the proposed structure was due to the larger MSE caused by the increasing tail-end AEC adaptive filter step sizes. After the BF sector switch the proposed AEC-BF-AEC structure's ERLE performance dropped but converged back to steady state faster as the tail-end AEC step size was increased, as seen in Figure 5.10. However, as the tail-end AEC step size was increased the final steady state ERLE 
performance achieved after re-convergence was increasingly lower. A summary of the average ERLE performance for all structures during initial steady state conditions, during re-convergence after the $\mathrm{BF}$ sector change, and during final steady state conditions is given in Table 5.8 below.

\begin{tabular}{|c|c|c|c|c|c|c|}
\cline { 2 - 6 } \multicolumn{1}{c|}{} & $\begin{array}{c}\text { AEC } \\
\text { Only }\end{array}$ & $\begin{array}{c}\text { BF- } \\
\text { AEC }\end{array}$ & $\begin{array}{r}\text { AEC- } \\
\mathbf{B F}\end{array}$ & $\begin{array}{r}\text { AEC-BF- } \\
\mathbf{A E C}\end{array}$ & $\begin{array}{r}\text { AEC-BF- } \\
\text { AEC }\end{array}$ & $\begin{array}{r}\text { AEC-BF- } \\
\text { AEC }\end{array}$ \\
\hline $\begin{array}{c}\text { Avg. ERLE during } \\
\text { initial steady state }\end{array}$ & 8.74 & 14.50 & 14.55 & 14.38 & 14.31 & 14.09 \\
\hline $\begin{array}{c}\text { Avg. ERLE during } \\
\text { re-convergence (dB) }\end{array}$ & 8.74 & 12.67 & 14.55 & 13.75 & 13.84 & 13.83 \\
\hline $\begin{array}{c}\text { Avg. ERLE during } \\
\text { finial steady state } \\
\text { (dB) }\end{array}$ & 8.74 & 14.47 & 14.55 & 14.48 & 14.08 & 13.86 \\
\hline
\end{tabular}

TABLE 5.8 Average ERLE Performance under Changing Sector Based BF Conditions with Increasing Tail-End AEC Step Sizes for Proposed Structure

During initial and final steady state operation the proposed structure's average ERLE performance lagged the BF-AEC and AEC-BF structures by at most $0.69 \mathrm{~dB}$ for both cases. The single microphone structure was outperformed by the proposed structure by at least $5.12 \mathrm{~dB}$ during both initial and final steady state operation.

During re-convergence the proposed structure's average ERLE performance lagged the AEC-BF structure's by at most $0.8 \mathrm{~dB}$ and lead the BF-AEC structure's by at least $1.08 \mathrm{~dB}$ on average, as shown in Table 5.8. Again, the single microphone structure was outperformed by the proposed structure by at least $5.01 \mathrm{~dB}$ during re-convergence. 


\subsubsection{Changing Sector Based BF Simulation Results Summary}

Taking the above simulation results into consideration, a summary of the trends observed on the ERLE performance of the proposed structure compared to current structures under changing sector based $\mathrm{BF}$ conditions is as follows:

- The ERLE performance of the AEC-BF structure was unaffected by the time variation of the sector based $\mathrm{BF}$ (see Figures $5.7-5.10$ )

- The single microphone AEC structure had the worst overall ERLE performance of all the structures and was unaffected by the BF sector change (see Figures $5.7-5.10$ )

- As the length of front-end AEC adaptive filters was increased the ERLE performance of the proposed structure improved towards that of AEC-BF structure's and outperformed the single microphone structure by at least 5.12dB (see Figures $5.7-$ 5.10 and Tables $5.5-5.6)$

- During re-convergence as the number of front-end AEC adaptive filter taps was increased the ERLE performance of the proposed structure improved and significantly outperformed the BF-AEC structure by at least $1.12 \mathrm{~dB}$ and the single microphone structure by at least $5.05 \mathrm{~dB}$ on average (see Figures $5.7-5.10$ and Tables 5.5-5.6)

- As the front-end and tail-end AEC adaptive filters step sizes were increased the initial and final steady state ERLE performance of the proposed structure degraded away from the performance of the AEC-BF and BF-AEC structures but still outperformed the single microphone structure by at least $4.76 \mathrm{~dB}$ (see Figures $5.9-5.10$ and Table 5.7) 
- During re-convergence increasing the front-end AEC adaptive filter step sizes did not help the proposed structure converge faster after the BF sector change (see Figure 5.9)

- During re-convergence increasing the tail-end AEC adaptive filter step sizes resulted in slightly faster convergence in ERLE performance for the proposed structure (see Figure 5.10)

Once more, simulations of the proposed structure were also performed for increasing front-end and tail-end AEC adaptive filter step sizes towards and beyond 2.0. At step sizes of 2.0 and greater the proposed structure's AEC adaptive filters became divergent as discussed in Section 3.5.1.

The slightly higher average steady state ERLE values attained by the BF-AEC and AECBF structures in Sections 5.2.1 and 5.3.1 respectively compared to the other combined structures could possibly be attributed to a varying beamformer beamshape with frequency, as well as loudspeaker distortion. 


\section{CHAPTER 6 SIMULATION OF STRUCTURES UNDER CHANGING ROOM CONDITIONS}

\subsection{Simulation of Structures under Changing Room and Fixed BF Conditions}

For the following changing room acoustic simulations two different sets of LRM IRs were used to create the artificial microphone signals for a six channel circular microphone array with beamforming fixed to sector one. The adaptation step size for the single microphone structure, the BF-AEC structure, and the AEC-BF structure was set to 0.2 for the artificial changing room simulations and to 0.3 for the real changing room simulations. The AEC adaptive filters of the aforementioned structures were set to fully adapt to 1000 tap models of the LRM transfer functions for both sets of simulations. The adaptation process was allowed to continue for 25000 iterations under the room acoustics created using the first set of LRM IRs. Then starting at the next iteration the room acoustics created using the second set of LRM IRs were applied and the structures were allowed to readapt for another 25000 iterations.

The following simulations demonstrated how the current and proposed structures were impacted when the acoustical room environment changed with respect to the microphone array. This situation would have arisen during hands-free communication when the 
microphone array was moved from one location to another or objects were placed or removed from the vicinity of the array.

\subsubsection{Simulation Results under an Artificially Changing Room Environment}

In order to create a changing room condition the LRM IRs of Figure 4.3 were drastically varied between taps $11-110$ in order to artificially simulate a change in the room environment in close proximity to the six channel circular microphone array. These specific taps were attenuated via multiplication with a sequence of uniformly distributed random numbers on the interval $(-1.0,1.0)$. Varying taps $11-110$ corresponded to the room environment changing acoustically between approximately $20 \mathrm{~cm}$ and $2.4 \mathrm{~m}$ with respect to the microphone array when sampling at $8 \mathrm{kHz}$ and when a nominal speed of sound in air of $350 \mathrm{~m} / \mathrm{s}$ was assumed. The remaining taps of the LRM IRs of Figure 4.3 were only slightly varied to indicate that the room environment remained approximately stationary outside the range indicated with respect to the microphone array. The modified LRM IRs of Figure 4.3 are shown in Figure 6.1 below. Simulation results under this artificially changing room condition are discussed below. 

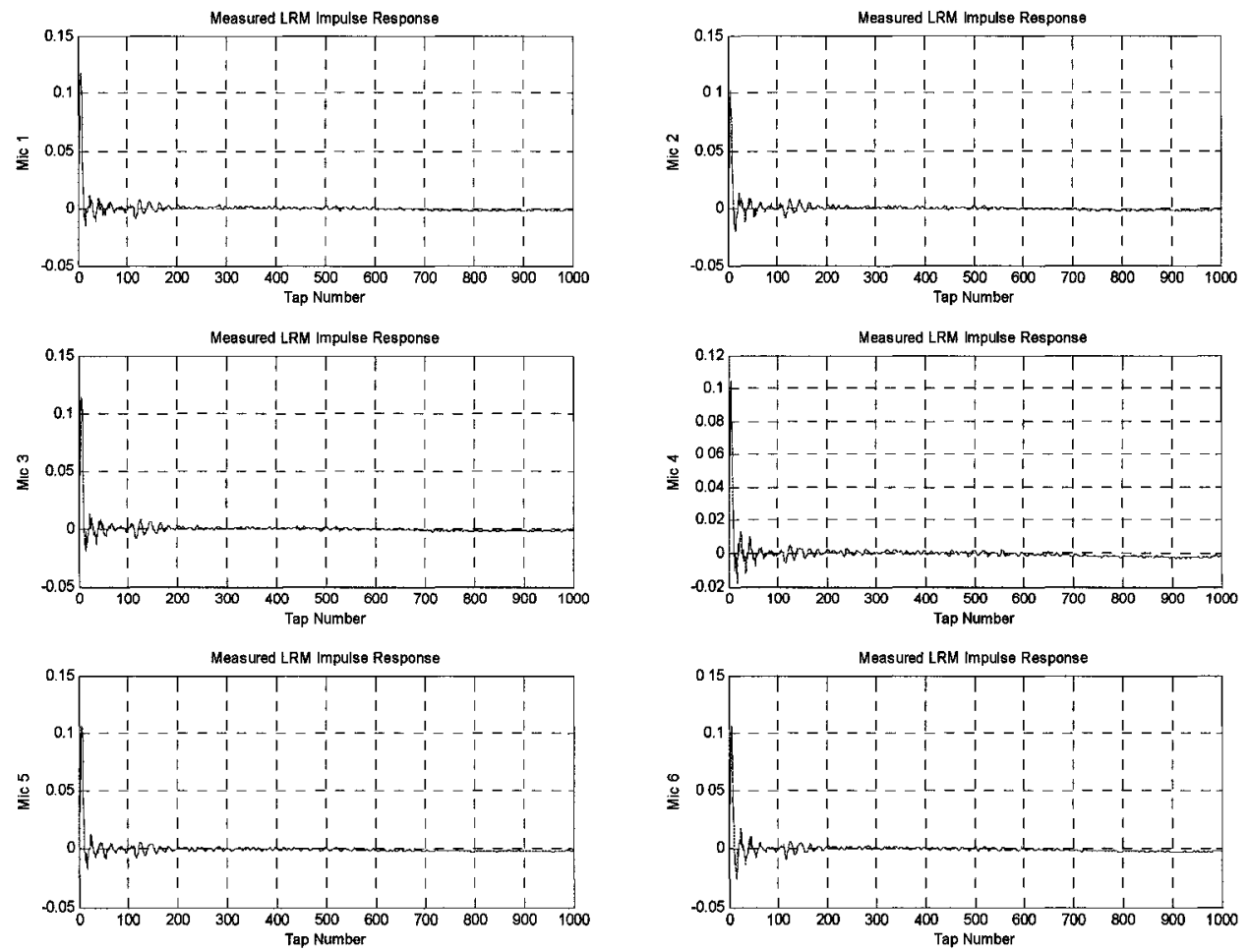

FIGURE 6.1 Modified LRM IRs used to Artificially Simulate Changing Room Acoustical Conditions

\subsubsection{Simulation Results under Increasing Front-End AEC Taps}

Figure 6.2 (shown below) displays ERLE simulation results for all current structures and for the proposed structure where the size of the front-end AECs (see Figure 3.4) were increased from 50 taps up to 100 taps while the front and tail-end AEC step sizes were held constant at 0.2 . 


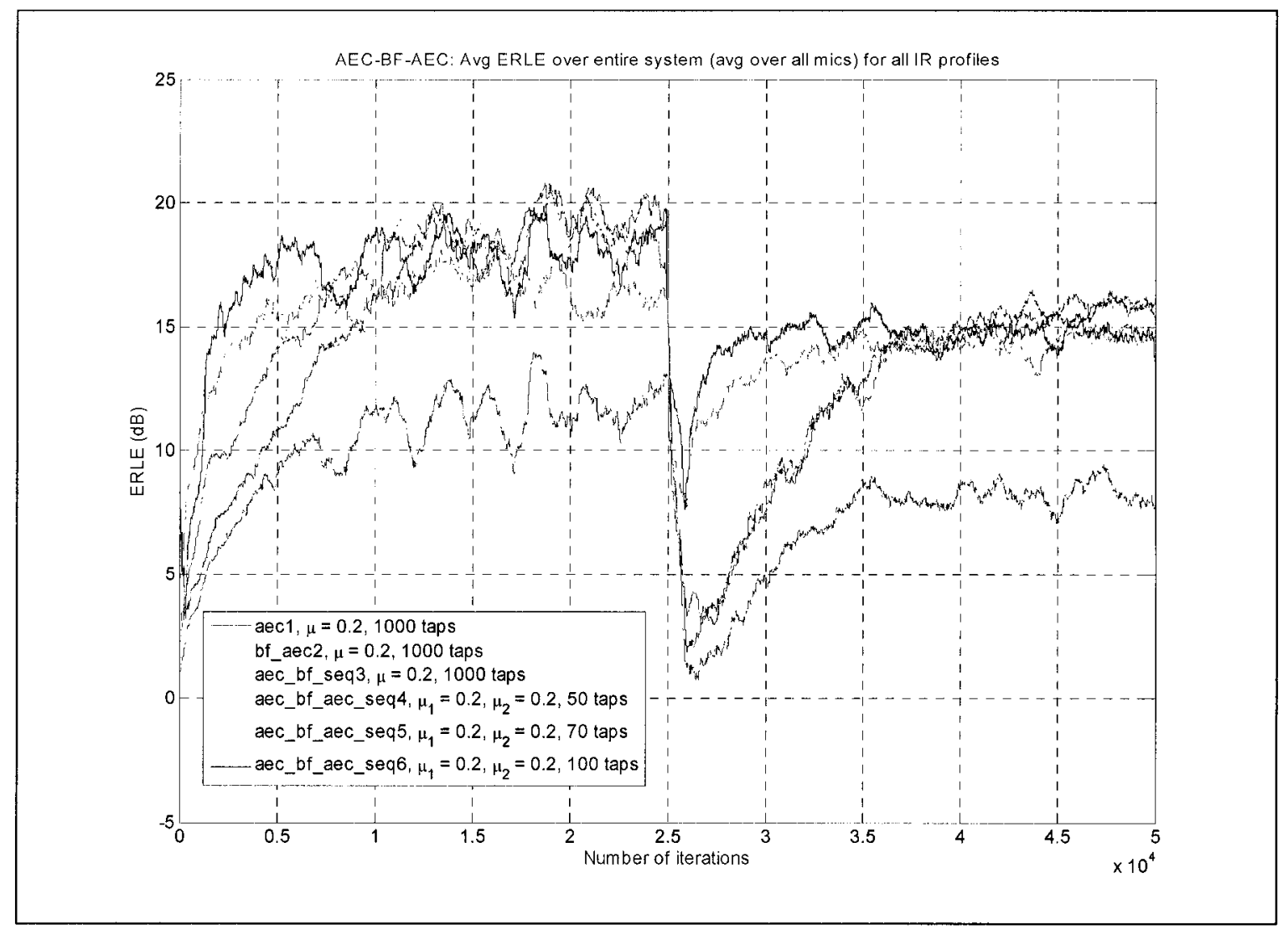

\section{FIGURE 6.2 ERLE Results under Artificially Changing Room Conditions with Increasing Front-End AEC Taps for Proposed Structure}

As shown in Figure 6.2 the single microphone structure, indicated by the purple curve, had the worst overall ERLE performance out of all the structures. The BF-AEC and AEC-BF structures attained similar overall ERLE performance as indicated by the blue and red curves respectively. The single microphone, BF-AEC, and AEC-BF structures ERLE performance suffered greatly when the change in room environment occurred but the proposed structure performed considerably better at every front-end AEC length from 50 to 70 and finally 100 , as shown by the green, yellow, and black curves respectively in Figure 6.2. The proposed structure's ERLE performance did not drop as much and converged significantly faster, as the number of front-end AEC taps increased compared to the BF-AEC and AEC-BF structures. This was a result of the ability of the proposed 
structure's relatively short front-end AECs to quickly track the changes in the LRM IRs and therefore re-converge faster to steady state ERLE as the length of the front-end AECs increased from 50 to 100 . Since the current structures modeled the entire 1000 tap LRM IRs they were not able to adapt to the changes in the LRM transfer functions as quickly as the proposed structure and thus their ERLE performance took longer to reach steady state.

As the number of front-end AEC taps increased for the AEC-BF-AEC structure the steady state ERLE performance improved towards that of the AEC-BF and BF-AEC structures before and after the change in room environment. Also, it should be noted that the proposed AEC-BF-AEC structure displayed faster initial convergence at all front-end AEC lengths during the first 7500 iterations than all of the current structures. A summary of the average ERLE performance for all structures during initial steady state conditions, during re-convergence after the change in LRM IRs, and during final steady state conditions is given in Table 6.1 below. All structures were assumed to be in initial steady state, re-convergence, and final steady state conditions between $12500-25000,25000-$ 37500 , and $37500-50000$ iterations respectively as seen in Figure 6.2. 


\begin{tabular}{|c|c|c|c|c|c|c|}
\cline { 2 - 6 } \multicolumn{1}{c|}{} & $\begin{array}{c}\text { AEC } \\
\text { Only }\end{array}$ & $\begin{array}{c}\text { BF- } \\
\text { AEC }\end{array}$ & $\begin{array}{c}\text { AEC- } \\
\text { BF }\end{array}$ & $\begin{array}{c}\text { AEC-BF- } \\
\text { AEC 50 } \\
\text { Taps }\end{array}$ & $\begin{array}{c}\text { AEC-BF- } \\
\text { AEC 70 } \\
\text { Taps }\end{array}$ & $\begin{array}{c}\text { AEC-BF- } \\
\text { AEC 100 } \\
\text { Taps }\end{array}$ \\
\hline $\begin{array}{r}\text { Avg. ERLE during } \\
\text { initial steady state } \\
\text { (dB) }\end{array}$ & 11.76 & 19.06 & 18.71 & 16.73 & 17.25 & 18.16 \\
\hline $\begin{array}{c}\text { Avg. ERLE during } \\
\text { re-convergence (dB) }\end{array}$ & 5.71 & 9.33 & 9.24 & 13.03 & 13.77 & 14.19 \\
\hline $\begin{array}{c}\text { Avg. ERLE during } \\
\text { finial steady state } \\
\text { (dB) }\end{array}$ & 8.23 & 15.24 & 15.37 & 14.45 & 14.63 & 14.70 \\
\hline
\end{tabular}

TABLE 6.1 Average ERLE Performance under Artificially Changing Room Conditions with Increasing Front-End AEC Taps for Proposed Structure

After the structures reached initial steady state the proposed structure's average ERLE performance using 100 front-end AEC taps lagged the BF-AEC and AEC-BF structures by less than a single $\mathrm{dB}$ and was more than $6 \mathrm{~dB}$ higher than the performance of the single microphone structure. At 50 and 70 front-end AEC taps the proposed structure's average ERLE performance trailed the BF-AEC and AEC-BF structures by about 2 and $1.5 \mathrm{~dB}$ respectively but was higher than the single microphone structure by approximately 5 and $5.5 \mathrm{~dB}$ respectively. During final steady state operation the same average ERLE trends for the proposed AEC-BF-AEC structure during initial steady state conditions were observed as shown in Table 6.1.

During re-convergence, after the change in the room environment occurred, the average ERLE value attained by the proposed AEC-BF-AEC structure using 100 front-end AEC taps was almost $5 \mathrm{~dB}$ greater than for the $\mathrm{BF}-\mathrm{AEC}$ and $\mathrm{AEC}-\mathrm{BF}$ structures and about $8.5 \mathrm{~dB}$ greater than the single microphone structure. At 50 and 70 front-end AEC taps the 
proposed structure's average ERLE performance was approximately 3.7 and $4.4 \mathrm{~dB}$ greater than the BF-AEC and AEC-BF structures and approximately 7.3 and $8 \mathrm{~dB}$ higher than the single microphone structure.

\subsubsection{Simulation Results under Increasing Front-End AEC Taps Towards Full Echo Path Modeling}

Figure 6.3 (see below) displays ERLE simulation results for all current structures and for the proposed structure where the size of the front-end AECs were increased from 80 taps towards full modeling of the individual LRM IRs while the front and tail-end AEC step sizes were held constant at 0.2 . 


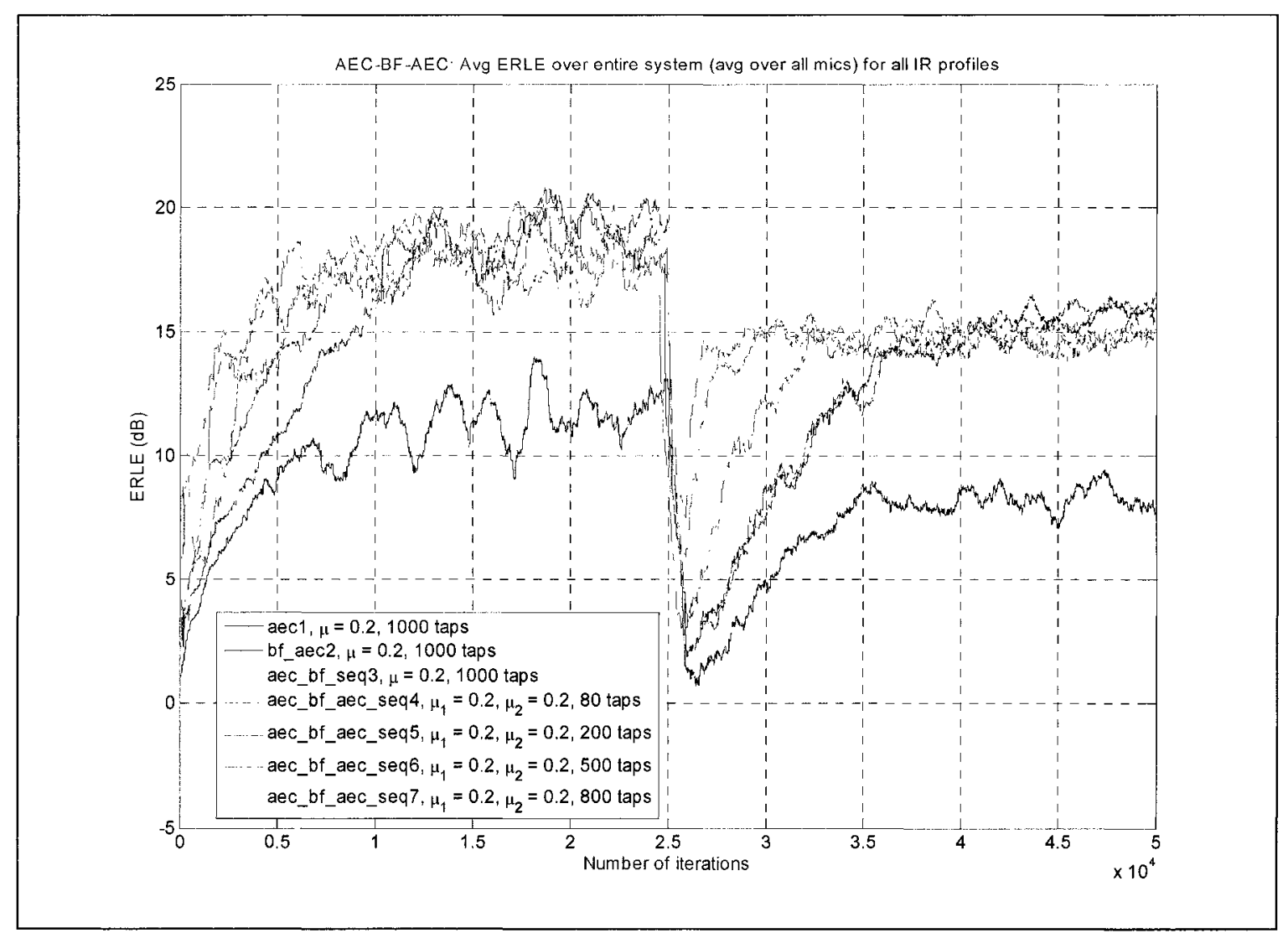

\section{FIGURE 6.3 ERLE Results under Artificially Changing Room Conditions with Increasing Front-End AEC Taps Towards Full Echo Path Modeling for Proposed Structure}

The single microphone, BF-AEC, and AEC-BF structures ERLE performance, indicated by the black, blue and red curves respectively, was the same as in Figure 6.2. As the number of front-end AEC taps increased for the proposed structure from 80 to 200 to 500 and finally to 800 the ERLE performance deteriorated towards that of the BF-AEC and AEC-BF structure after the change in room environment occurred, as shown by the green, cyan, purple, and yellow curves respectively in Figure 6.3. This was a result of the inability of the proposed structure's longer front-end AECs to track the changes in the LRM IRs as quickly as when shorter front-end AECs were used (see Figure 6.2). Therefore, the proposed structure re-converged slower to steady state ERLE as the length of the front-end AECs increased from 80 to 800 . 
As the number of front-end AEC taps increased from 80 to 800 for the AEC-BF-AEC structure the steady state ERLE performance was approximately the same as that of the AEC-BF and BF-AEC structures before and after the change in room environment. A summary of the average ERLE performance for all structures during initial steady state conditions, during re-convergence after the change in LRM IRs, and during final steady state conditions is given in Table 6.2 below. All structures were assumed to be in initial steady state, re-convergence, and final steady state conditions between $12500-25000$, $25000-37500$, and $37500-50000$ iterations respectively as seen in Figure 6.3.

\begin{tabular}{|c|c|c|c|c|c|c|c|}
\hline & $\begin{array}{l}\text { AEC } \\
\text { Only }\end{array}$ & $\begin{array}{l}\mathrm{BF}- \\
\mathrm{AEC}\end{array}$ & AEC & $\begin{array}{l}\text { AEC- } \\
\text { BF- } \\
\text { AEC 80 } \\
\text { Taps }\end{array}$ & $\begin{array}{c}\text { AEC- } \\
\text { BF- } \\
\text { AEC } \\
200 \\
\text { Taps }\end{array}$ & $\begin{array}{r}\text { AEC- } \\
\text { BF } \\
\text { AEC } \\
\mathbf{5 0 0} \\
\text { Taps }\end{array}$ & $\begin{array}{l}\text { AEC- } \\
\text { BF- } \\
\text { AEC } \\
\mathbf{8 0 0} \\
\text { Taps }\end{array}$ \\
\hline $\begin{array}{l}\text { Avg. ERLE } \\
\text { during initial } \\
\text { steady state (dB) }\end{array}$ & 11.76 & 19.06 & 18.71 & 17.34 & 18.31 & 18.05 & 17.50 \\
\hline $\begin{array}{l}\text { Avg. ERLE } \\
\text { during re- } \\
\text { convergence } \\
\text { (dB) }\end{array}$ & 5.71 & 9.33 & 9.24 & 13.96 & 13.84 & 11.59 & 10.59 \\
\hline $\begin{array}{l}\text { Avg ERLE } \\
\text { during finial } \\
\text { steady state (dB) }\end{array}$ & 8.23 & 15.24 & 15.37 & 14.77 & 14.65 & 14.98 & 15.21 \\
\hline
\end{tabular}

TABLE 6.2 Average ERLE Performance under Artificially Changing Room Conditions with Increasing Front-End AEC Taps Towards Full Echo Path Modeling for Proposed Structure

During initial and final steady state operation the proposed AEC-BF-AEC structure achieved approximately the same average ERLE performance at all front-end AEC tap lengths as the BF-AEC and AEC-BF structures as seen in Table 6.2. The single AEC 
structure was outperformed by the proposed structure at all front-end tap lengths during both initial and final steady state operation by at least 5.5 and $6 \mathrm{~dB}$ respectively on average, as shown in Table 6.2.

During re-convergence, after the change in the room environment occurred, the average ERLE value attained by the proposed AEC-BF-AEC structure using 80 and 200 front-end AEC taps was about $4.5 \mathrm{~dB}$ greater than for the BF-AEC and AEC-BF structures and about $8 \mathrm{~dB}$ greater than the single microphone structure. At 500 and 800 front-end AEC taps the proposed structure's average ERLE performance was only approximately 2.2 and $1.2 \mathrm{~dB}$ greater than the BF-AEC and AEC-BF structures and approximately 5.9 and $4.9 \mathrm{~dB}$ higher than the single microphone structure.

\subsubsection{Simulation Results under Increasing Front-End AEC Step Size}

Figure 6.4 (shown below) displays ERLE simulation results for all current structures and for the proposed structure where the front-end AECs adaptation step sizes were increased from 0.1 to 0.4 while the tail-end AEC adaptation step size was held constant at 0.2 . Also, the front-end AECs adaptive filter lengths were held constant at 80 taps. 


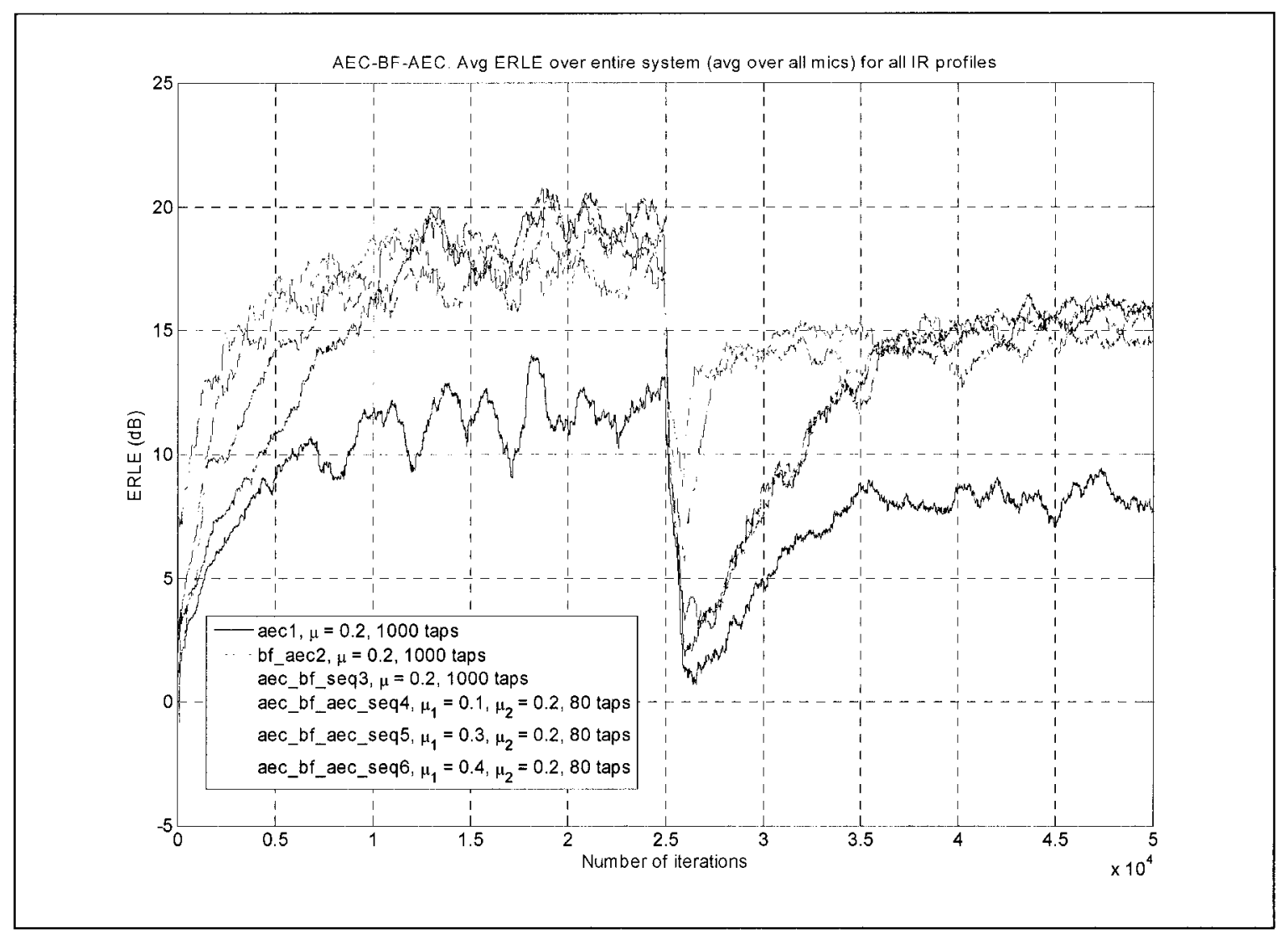

\section{FIGURE 6.4 ERLE Results under Artificially Changing Room Conditions with Increasing Front-End AEC Step Sizes for Proposed Structure}

The single microphone, BF-AEC, and AEC-BF structures ERLE performance, indicated by the black, blue and red curves respectively, was the same as in Figure 6.2. The overall steady state ERLE performance of the proposed AEC-BF-AEC structure degraded, before and after the change in the LRM IRs occurred, as the front-end AECs step sizes increased from 0.1 to 0.3 and finally 0.4 , as indicated by the green, purple, and yellow curves respectively. As the front-end AECs step sizes, $\mu_{l}$, were increased the proposed structure initially converged faster than the other structures but to a lower steady state ERLE due to higher MSE caused by the larger adaptation step sizes. 
After the change in room conditions was applied the proposed structures ERLE performance suffered considerably less, at all values of $\mu_{1}$, than the other structures as a result of its ability track the severe changes in the LRM IRs quickly due to the relatively short front-end AECs adaptive filters. As $\mu_{1}$ increased the proposed structure was able to initially track the major changes in the LRM IRs faster, due to the higher adaptation step size, resulting in a lower drop and faster convergence in ERLE performance than at lower values of $\mu_{1}$ (see Figure 6.4). However, the larger front-end AECs step sizes of the proposed structure resulted in lower final steady state ERLE performance due to higher MSE. A summary of the average ERLE performance for all structures during initial steady state conditions, during re-convergence after the change in LRM IRs, and during final steady state conditions is given in Table 6.3 below. All structures were assumed to be in initial steady state, re-convergence, and final steady state conditions between 12500 $-25000,25000-37500$, and $37500-50000$ iterations respectively as seen in Figure 6.4.

\begin{tabular}{|c|c|c|c|c|c|c|}
\cline { 2 - 7 } \multicolumn{1}{c|}{} & $\begin{array}{c}\text { AEC } \\
\text { Only }\end{array}$ & $\begin{array}{c}\text { BEC } \\
\text { AEC }\end{array}$ & $\begin{array}{r}\text { AEC- } \\
\text { BF }\end{array}$ & $\begin{array}{r}\text { AEC-BF- } \\
\text { AEC } \\
\boldsymbol{\mu}_{1}=\mathbf{0 . 1}\end{array}$ & $\begin{array}{r}\text { AEC-BF- } \\
\text { AEC } \\
\boldsymbol{\mu}_{1}=\mathbf{0 . 3}\end{array}$ & $\begin{array}{r}\text { AEC-BF- } \\
\text { AEC } \\
\boldsymbol{\mu}_{\mathbf{1}}=\mathbf{0 . 4}\end{array}$ \\
\hline $\begin{array}{c}\text { Avg. ERLE during } \\
\text { initial steady state } \\
\text { (dB) }\end{array}$ & 11.76 & 19.06 & 18.71 & 18.03 & 17.07 & 16.34 \\
\hline $\begin{array}{c}\text { Avg. ERLE during } \\
\text { re-convergence (dB) }\end{array}$ & 5.71 & 9.33 & 9.24 & 13.64 & 13.56 & 13.05 \\
\hline $\begin{array}{c}\text { Avg. ERLE during } \\
\text { finial steady state }\end{array}$ & 8.23 & 15.24 & 15.37 & 15.32 & 14.37 & 13.61 \\
\hline \begin{tabular}{c} 
(dB) \\
\hline
\end{tabular}
\end{tabular}

TABLE 6.3 Average ERLE Performance under Artificially Changing Room Conditions with Increasing Front-End AEC Step Sizes for Proposed Structure 
During initial and final steady state operation the proposed AEC-BF-AEC structure's average ERLE performance closely matched that of the BF-AEC and AEC-BF structure when the front-end AECs step sizes were 0.1, as shown in Table 6.3. As the front-end AECs step sizes increased to 0.4 the average ERLE performance of the proposed structure decreased by about $1.7 \mathrm{~dB}$ during initial and final steady state operation. As the front-end AECs adaptation step sizes increased the proposed structure's average ERLE performance was between 6.3 and $4.6 \mathrm{~dB}$ higher than the single microphone structure during initial steady state operation. During final steady state operation the proposed structure performed better than the single microphone structure by 7.1 to $5.4 \mathrm{~dB}$ as the front-end AECs step sizes increased from 0.1 to 0.4 , as shown in Table 6.3.

During re-convergence, after the change in the room environment occurred, the average ERLE value attained by the proposed AEC-BF-AEC structure, as the front-end AECs adaptation step sizes increased from 0.1 to 0.4 , was between approximately 4.3 and $3.7 \mathrm{~dB}$ higher than for the BF-AEC and AEC-BF structures. The single microphone structure was outperformed by the proposed structure by about 8 to $7.4 \mathrm{~dB}$ as the front-end AECs adaptive filters step sizes were increased from 0.1 to 0.4 , as seen in Table 6.3

\subsubsection{Simulation Results under Increasing Tail-End AEC Step Size}

Figure 6.5 (shown below) displays ERLE simulation results for all current structures and for the proposed structure where the tail-end AEC adaptation step size was increased from 0.1 to 0.3 while the front-end AECs adaptation step sizes were held constant at 0.2 . Also, the front-end AECs adaptive filter lengths were held constant at 60 taps. 


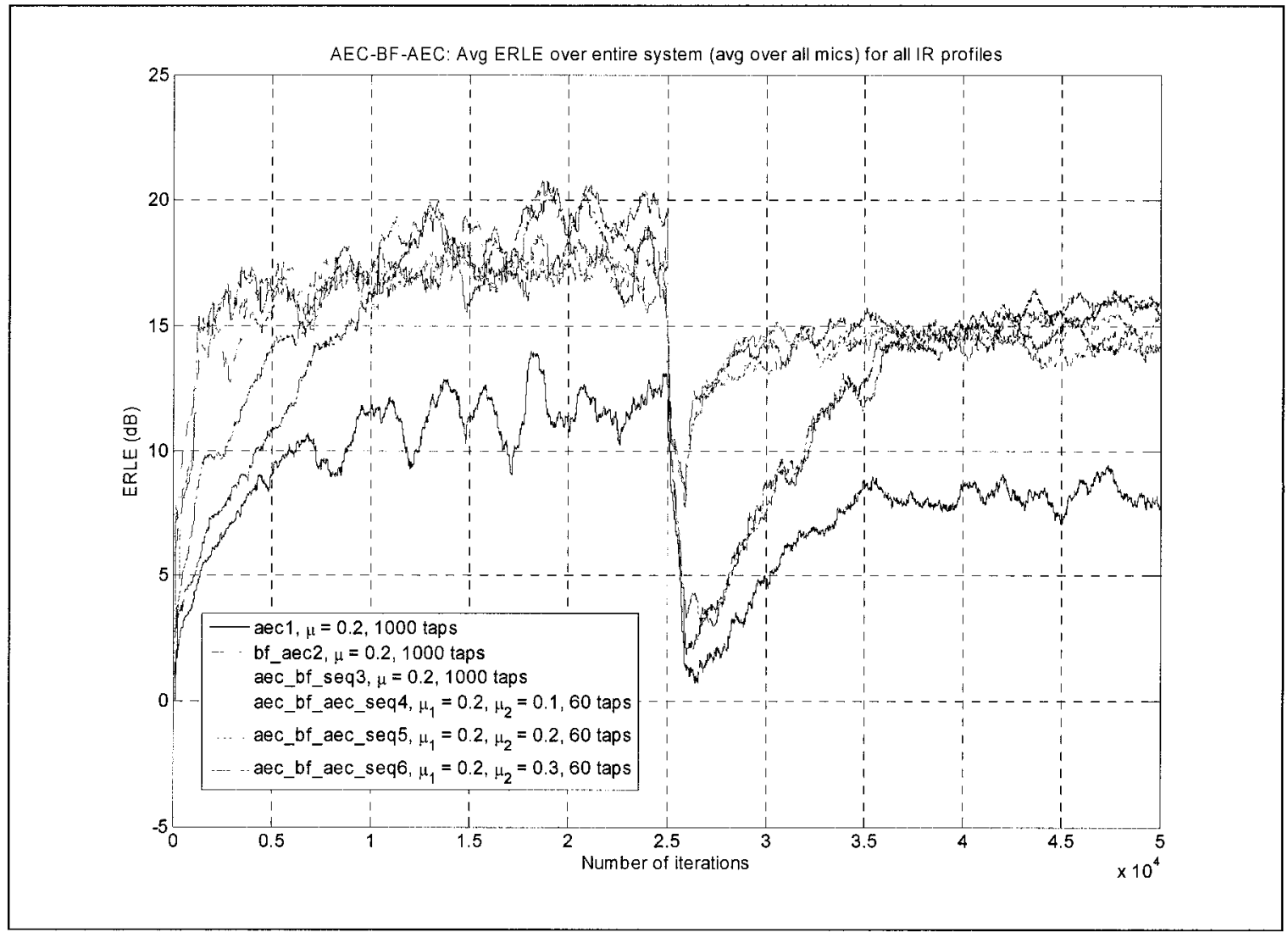

FIGURE 6.5 ERLE Results under Artificially Changing Room Conditions with Increasing Tail-End AEC Step Sizes for Proposed Structure

The single microphone, BF-AEC, and AEC-BF structures ERLE performance, indicated by the black, blue and red curves respectively, was the same as in Figure 6.2. The overall steady state ERLE performance of the proposed AEC-BF-AEC structure slightly degraded, before and after the change in the LRM IRs occurred, as the tail-end AEC step size increased from 0.1 to 0.2 and finally 0.3 , as indicated by the green, purple, and cyan curves respectively. As the tail-end AEC step sizes, $\mu_{2}$, were increased the proposed structure initially converged faster than the other structures but to a lower steady state ERLE due to higher MSE caused by the larger adaptation step sizes. 
After the change in room conditions was applied the proposed structure's ERLE performance suffered considerably less, at all values of $\mu_{2}$, than the other structures as a result of its ability track the severe changes in the LRM IRs quickly due to the relatively short front-end AECs adaptive filters. As $\mu_{2}$ increased the proposed structure was able to initially track the changes in the LRM IRs faster, due to the higher adaptation step size, resulting in faster convergence in ERLE performance than at lower values of $\mu_{2}$ (see Figure 6.5). However, the larger tail-end AEC step sizes of the proposed structure resulted in lower final steady state ERLE performance due to higher MSE. A summary of the average ERLE performance for all structures during initial steady state conditions, during re-convergence after the change in LRM IRs, and during final steady state conditions is given in Table 6.4 below. All structures were assumed to be in initial steady state, re-convergence, and final steady state conditions between $12500-25000,25000-$ 37500 , and $37500-50000$ iterations respectively as seen in Figure 6.5 .

\begin{tabular}{|c|c|c|c|c|c|c|}
\cline { 2 - 6 } \multicolumn{1}{c|}{} & $\begin{array}{r}\text { AEC } \\
\text { Only }\end{array}$ & $\begin{array}{c}\text { BE- } \\
\text { AEC }\end{array}$ & $\begin{array}{r}\text { AEC- } \\
\text { BF }\end{array}$ & $\begin{array}{r}\text { AEC-BF- } \\
\text { AEC } \\
\mu_{2}=\mathbf{0 . 1}\end{array}$ & $\begin{array}{c}\text { AEC-BF- } \\
\text { AEC } \\
\boldsymbol{\mu}_{2}=\mathbf{0 . 2}\end{array}$ & $\begin{array}{c}\text { AEC-BF- } \\
\text { AEC } \\
\boldsymbol{\mu}_{2}=\mathbf{0 . 3}\end{array}$ \\
\hline $\begin{array}{r}\text { Avg. ERLE during } \\
\text { initial steady state } \\
\text { (dB) }\end{array}$ & 11.76 & 19.06 & 18.71 & 17.27 & 17.14 & 17.25 \\
\hline $\begin{array}{c}\text { Avg. ERLE during } \\
\text { re-convergence (dB) }\end{array}$ & 5.71 & 9.33 & 9.24 & 13.38 & 13.83 & 13.78 \\
\hline $\begin{array}{c}\text { Avg. ERLE during } \\
\text { finial steady state } \\
\text { (dB) }\end{array}$ & 8.23 & 15.24 & 15.37 & 14.91 & 14.57 & 14.19 \\
\hline
\end{tabular}

TABLE 6.4 Average ERLE Performance under Artificially Changing Room Conditions with Increasing Tail-End AEC Step Sizes for Proposed Structure 
During initial steady state operation the proposed AEC-BF-AEC structure's average ERLE performance slightly decreased as the tail-end AEC step sizes increased and trailed the BF-AEC and AEC-BF structures by at most $1.92 \mathrm{~dB}$. The decrease in the proposed structure's average steady state ERLE performance was more evident during final steady state operation as the tail-end AEC step sizes increased and trailed the BF-AEC and AEC-BF structures by no more than $1.2 \mathrm{~dB}$. As the tail-end AEC adaptation step sizes increased the proposed structure's average ERLE performance was between 5.5 and 5.4dB higher than the single microphone structure during initial steady state operation. During final steady state operation the proposed structure performed better than the single microphone structure by 6.7 to $6.0 \mathrm{~dB}$ as the tail-end AEC step sizes increased from 0.1 to 0.3 , as shown in Table 6.4 .

During re-convergence, after the change in the room environment occurred, the average ERLE value attained by the proposed AEC-BF-AEC structure, as the tail-end AEC adaptation step sizes increased from 0.1 to 0.3 , was between approximately 4.1 and $4.6 \mathrm{~dB}$ higher than for the BF-AEC and AEC-BF structures. The single microphone structure was outperformed by the proposed structure by about 7.7 to $8.1 \mathrm{~dB}$ as the tail-end AEC adaptive filters step sizes were increased from 0.1 to 0.3 , as seen in Table 6.4

Also, it should be noted that the impact on average initial and final steady state ERLE performance for the proposed structure was greater when the front-end AECs step sizes were increased compared to increasing the tail-end AEC step sizes. As the front-end AECs step sizes were increased from 0.1 to 0.4 the average initial and final steady state 
ERLE for the proposed structure dropped by approximately $1.7 \mathrm{~dB}$ (see Table 6.3). The average initial and final steady state ERLE dropped by only 0.14 and $0.72 \mathrm{~dB}$ respectively when the tail-end AEC step sizes were increased (see Table 6.4).

\subsubsection{Artificially Changing Room Environment Simulation Results Summary}

Taking the above simulation results into consideration, a summary of the trends observed on the ERLE performance of the proposed structure compared to current structures under artificially changing room conditions is as follows:

- As the length of front-end AEC adaptive filters was increased the initial and final steady state ERLE performance of the proposed structure improved towards that of the BF-AEC and AEC-BF structures and outperformed the single microphone structure by at least $5 \mathrm{~dB}$ (see Figures $6.2-6.3$ and Tables $6.1-6.2$ )

- The initial and final steady state ERLE performance of the proposed structure using relatively short front-end AEC adaptive filter lengths between 50 and 100 performed similarly to the BF-AEC and AEC-BF structures (see Figure 6.2 and Table 6.1)

- During re-convergence as the number of front-end AEC adaptive filter taps was increased between 50 and 100 the ERLE performance of the proposed structure improved and significantly outperformed the BF-AEC and AEC-BF structures by up to approximately $5 \mathrm{~dB}$ and the single microphone structure by at least $7 \mathrm{~dB}$ on average(see Figure 6.2 and Table 6.1)

- As the length of front-end AEC adaptive filters was increased beyond 100 taps the ERLE performance of the proposed structure, after the change in room conditions, 
degraded towards that of the BF-AEC and AEC-BF structures (see Figures $6.2-6.3$ and Tables $6.1-6.2$ )

- As the front-end and tail-end AEC adaptive filters step sizes were increased the initial and final steady state ERLE performance of the proposed structure degraded away from the performance of the $\mathrm{BF}-\mathrm{AEC}$ and $\mathrm{AEC}-\mathrm{BF}$ structures but still outperformed the single microphone structure by at least $4.6 \mathrm{~dB}$ (see Figures $6.4-6.5$ and Table 6.3)

- During re-convergence increasing the front-end AECs adaptive filter step sizes resulted in a lower drop and faster convergence in ERLE performance for the proposed structure compared to the other structures (see Figure 6.4)

- During re-convergence increasing the tail-end AEC adaptive filter step sizes resulted in faster convergence in ERLE performance for the proposed structure compared to the other structures (see Figure 6.5)

- Increasing the front-end AECs adaptive filters step sizes for the proposed structure had a greater negative impact on steady state ERLE performance than increasing the tail-end AEC adaptive filter step sizes (see Figures $6.4-6.5$ and Tables $6.3-6.4$ )

- The single microphone AEC structure had the worst overall ERLE performance of all the structures (see Figures 6.2-6.5)

Simulations of the proposed structure were also performed for increasing front-end and tail-end AEC adaptive filter step sizes towards and beyond 2.0. At step sizes of 2.0 and greater the proposed structure's AEC adaptive filters became divergent as discussed in Section 3.5.1. 


\subsubsection{Simulation Results under a Real Changing Room Environment}

The following changing room environment simulations were performed using the measured LRM IRs of Figure 4.5 and Figure 4.6 that were acquired under different room conditions as discussed in Section 4.4.2. The measured LRM IRs were used in the simulations of all structures in order to observe their behavior under the real world changing room condition described in section 4.4.2. Simulation results under this real world changing room condition are discussed below.

\subsubsection{Simulation Results under Increasing Front-End AEC Taps}

Figure 6.6 (shown below) displays ERLE simulation results for all current structures and for the proposed structure where the size of the front-end AECs (see Figure 3.4) were increased from 60 taps up to 100 taps while the front and tail-end AEC step sizes were held constant at 0.15 and 0.3 respectively. 


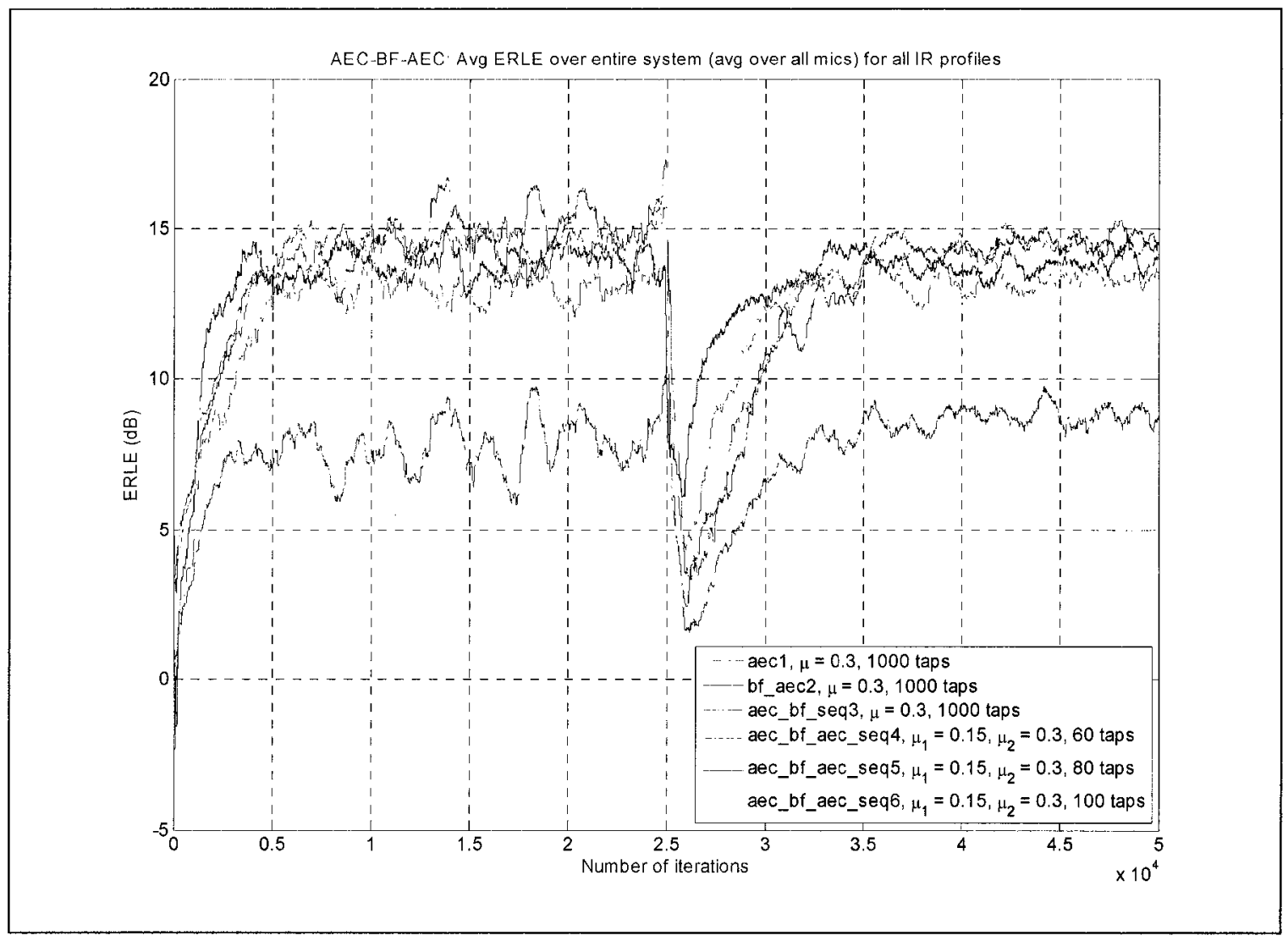

\section{FIGURE 6.6 ERLE Results under Real Changing Room Conditions with Increasing Front- End AEC Taps for Proposed Structure}

As seen in Figure 6.6 the same general trends occurred in the ERLE performance of all structures as under the artificially changing room conditions for increasing front-end AECs lengths (see Figure 6.2), as discussed in Section 6.1.1. Again, the BF-AEC and AEC-BF structures attained similar overall ERLE performance and the single microphone structure performed the worst, indicated by the blue, red, and purple curves respectively. The single microphone, BF-AEC, and AEC-BF structures ERLE performance suffered greatly when the change in room environment occurred but the proposed structure performed increasingly better as the front-end AEC length was increased from 60 to 80 and finally 100, as shown by the green, black, and yellow curves 
respectively in Figure 6.6. A summary of the average ERLE performance for all structures during initial steady state conditions, during re-convergence after the change in LRM IRs, and during final steady state conditions is given in Table 6.5 below. All structures were assumed to be in initial steady state, re-convergence, and final steady state conditions between $7500-25000,25000-37500$, and $37500-50000$ iterations respectively as seen in Figure 6.6.

\begin{tabular}{|c|c|c|c|c|c|c|}
\cline { 2 - 6 } \multicolumn{1}{c|}{} & $\begin{array}{c}\text { AEC } \\
\text { Only }\end{array}$ & $\begin{array}{c}\text { BF- } \\
\text { AEC }\end{array}$ & $\begin{array}{c}\text { AEC- } \\
\text { BF }\end{array}$ & $\begin{array}{c}\text { AEC-BF- } \\
\text { AEC 60 } \\
\text { Taps }\end{array}$ & $\begin{array}{r}\text { AEC-BF- } \\
\text { AEC 80 } \\
\text { Taps }\end{array}$ & $\begin{array}{c}\text { AEC-BF- } \\
\begin{array}{c}\text { AEC 100 } \\
\text { Taps }\end{array}\end{array}$ \\
\hline $\begin{array}{r}\text { Avg. ERLE during } \\
\text { initial steady state } \\
\text { (dB) }\end{array}$ & 7.79 & 14.82 & 14.59 & 13.08 & 13.89 & 14.15 \\
\hline $\begin{array}{c}\text { Avg. ERLE during } \\
\text { re-convergence (dB) }\end{array}$ & 6.50 & 10.40 & 10.70 & 11.35 & 12.47 & 12.60 \\
\hline $\begin{array}{c}\text { Avg. ERLE during } \\
\text { finial steady state } \\
\text { (dB) }\end{array}$ & 8.78 & 14.43 & 14.44 & 13.25 & 13.75 & 13.92 \\
\hline
\end{tabular}

\section{TABLE 6.5 Average ERLE Performance under Real Changing Room Conditions with} Increasing Front-End AEC Taps for Proposed Structure

Again, the same general trends for the average ERLE performance of all structures occurred in Table 6.4 as in Table 6.1 that was discussed in Section 6.1.1. The proposed structure performed about as well as the BF-AEC and AEC-BF structures during initial and final steady state operation at 100 front-end AEC taps and trailed by no more than $1.74 \mathrm{~dB}$ at 60 front-end AEC taps in either case. The proposed structure's average initial and final steady state ERLE was between 5.29 and 6.36dB higher and between 4.47 and 5.14dB higher as the number of front-end AEC taps increased correspondingly, than the single microphone AEC structure. 
During re-convergence the proposed structure's average ERLE performance was at least 0.65 to $1.9 \mathrm{~dB}$ higher than the BF-AEC and AEC-BF structures, as the number of frontend AEC taps increased correspondingly. Also, the proposed AEC-BF-AEC structure outperformed the single microphone AEC structure by 4.85 to $6.1 \mathrm{~dB}$ on average as the number of front-end AEC taps increased.

\subsubsection{Simulation Results under Increasing Front-End AEC Taps Towards Full Echo Path Modeling}

Figure 6.7 (see below) displays ERLE simulation results for all current structures and for the proposed structure where the size of the front-end AECs were increased from 80 taps towards full modeling of the individual LRM IRs while the front and tail-end AEC step sizes were held constant at 0.15 and 0.3 respectively. 


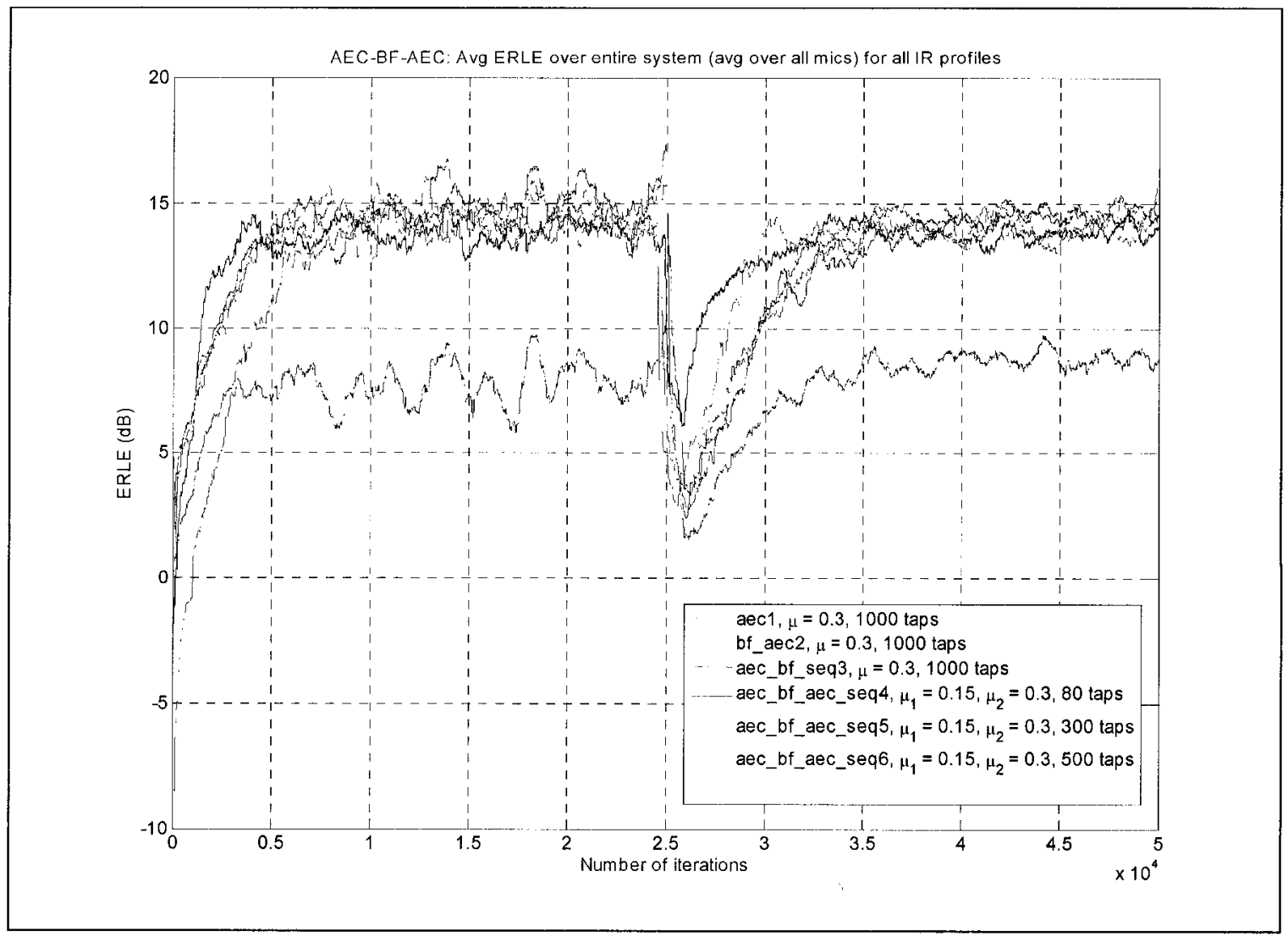

\section{FIGURE 6.7 ERLE Results under Real Changing Room Conditions with Increasing Front- End AEC Taps Towards Full Echo Path Modeling for Proposed Structure}

The single microphone, BF-AEC, and AEC-BF structures ERLE performance, indicated by the green, blue and red curves respectively, was the same as in Figure 6.6. Again, the same general trends occurred in the ERLE performance for the proposed structure as under the artificially changing room conditions for increasing front-end AECs lengths towards full modeling of the echo paths (see Figure 6.3), as discussed in Section 6.1.1. As the number of front-end AEC taps increased for the proposed structure from 80 to 300 and finally to 500 the ERLE performance deteriorated towards that of the BF-AEC and AEC-BF structure after the change in room environment occurred, as shown by the black, cyan, and purple, curves respectively in Figure 6.7. During steady state ERLE operation the performance of the proposed was approximately the same as that of the AEC-BF and 
BF-AEC structures at all front-end AEC tap lengths. A summary of the average ERLE performance for all structures during initial steady state conditions, during reconvergence after the change in LRM IRs, and during final steady state conditions is given in Table 6.6 below.

\begin{tabular}{|c|c|c|c|c|c|c|}
\cline { 2 - 6 } \multicolumn{1}{c|}{} & $\begin{array}{c}\text { AEC } \\
\text { Only }\end{array}$ & BEC & $\begin{array}{c}\text { AEC- } \\
\text { BF }\end{array}$ & $\begin{array}{c}\text { AEC-BF- } \\
\text { AEC 80 } \\
\text { Taps }\end{array}$ & $\begin{array}{r}\text { AEC-BF- } \\
\text { AEC 300 } \\
\text { Taps }\end{array}$ & $\begin{array}{c}\text { AEC-BF- } \\
\text { AEC 500 } \\
\text { Taps }\end{array}$ \\
\hline $\begin{array}{c}\text { Avg. ERLE during } \\
\text { initial steady state } \\
\text { (dB) }\end{array}$ & 7.79 & 14.82 & 14.59 & 13.89 & 14.31 & 14.00 \\
\hline $\begin{array}{c}\text { Avg. ERLE during } \\
\text { re-convergence (dB) }\end{array}$ & 6.50 & 10.40 & 10.70 & 12.47 & 11.75 & 10.18 \\
\hline $\begin{array}{c}\text { Avg. ERLE during } \\
\text { finial steady state } \\
\text { (dB) }\end{array}$ & 8.78 & 14.43 & 14.44 & 13.75 & 14.13 & 13.98 \\
\hline
\end{tabular}

TABLE 6.6 Average ERLE Performance under Real Changing Room Conditions with Increasing Front-End AEC Taps Towards Full Echo Path Modeling for Proposed Structure

Again, the same general trends for the average ERLE performance of all structures occurred in Table 6.6 as in Table 6.2 that was discussed in Section 6.1.1. During initial and final steady state operation the proposed AEC-BF-AEC structure achieved approximately the same average ERLE performance at all front-end AEC tap lengths as the BF-AEC and AEC-BF structures as seen in Table 6.6. The single AEC structure was outperformed by the proposed structure at all front-end tap lengths during both initial and final steady state operation by at least 6.1 and $4.97 \mathrm{~dB}$ respectively on average, as shown in Table 6.6 . 
During re-convergence the proposed structure's average ERLE performance ranged from at least $1.77 \mathrm{~dB}$ higher to about the same performance as the BF-AEC and $\mathrm{AEC}-\mathrm{BF}$ structures, as the number of front-end AEC taps increased correspondingly. Also, the proposed AEC-BF-AEC structure outperformed the single microphone AEC structure by 5.97 to $3.68 \mathrm{~dB}$ on average as the number of front-end AEC taps increased towards full modeling of the LRM IRs.

\subsubsection{Simulation Results under Increasing Front-End AEC Step Size}

Figure 6.8, shown below, displays ERLE simulation results for all current structures and for the proposed structure where the front-end AECs adaptation step sizes were increased from 0.1 to 0.3 while the tail-end AEC adaptation step size was held constant at 0.3 . Also, the front-end AECs adaptive filter lengths were held constant at 80 taps. 


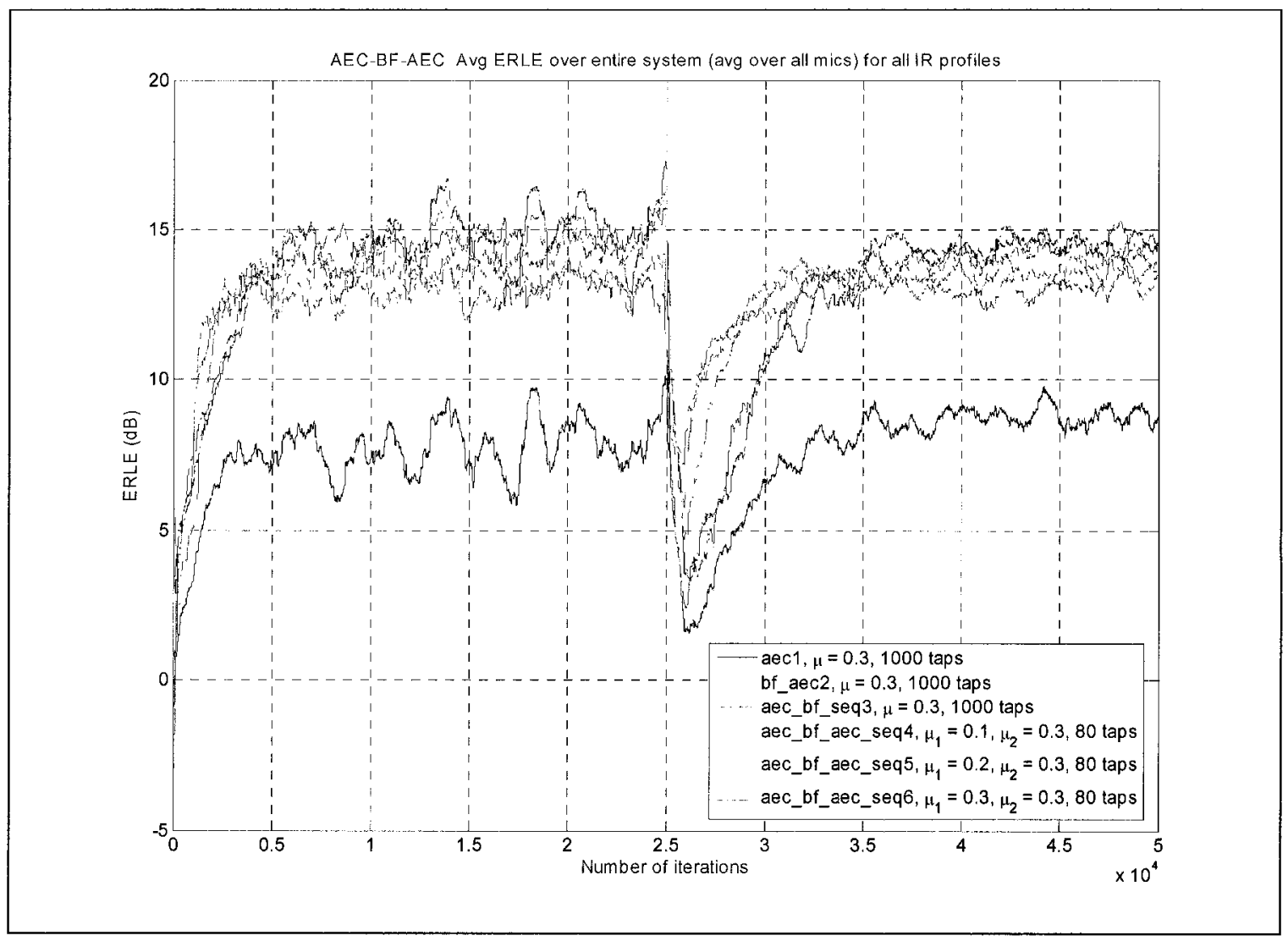

\section{FIGURE 6.8 ERLE Results under Real Changing Room Conditions with Increasing Front- End AEC Step Sizes for Proposed Structure}

The single microphone, BF-AEC, and AEC-BF structures ERLE performance, indicated by the black, blue and red curves respectively, was the same as in Figure 6.6. Again, the same trends occurred in the ERLE performance for the proposed structure as under the artificially changing room conditions for increasing front-end AECs step sizes (see Figure 6.4), as discussed in Section 6.1.1. The overall initial and final steady state ERLE performance of the proposed AEC-BF-AEC structure degraded as the front-end AECs step sizes increased, as indicated by the green, cyan, and purple curves respectively. As the front-end AECs step sizes increased the ERLE performance of the proposed structure dropped less and converged faster, but to lower steady state values, than the BF-AEC and AEC-BF structures after the change in room condition occurred. A summary of the 
average ERLE performance for all structures during initial steady state conditions, during re-convergence after the change in LRM IRs, and during final steady state conditions is given in Table 6.7 below.

\begin{tabular}{|c|c|c|c|c|c|c|}
\cline { 2 - 6 } \multicolumn{1}{c|}{} & $\begin{array}{c}\text { AEC } \\
\text { Only }\end{array}$ & $\begin{array}{c}\text { BF- } \\
\text { AEC }\end{array}$ & $\begin{array}{r}\text { AEC- } \\
\text { BF }\end{array}$ & $\begin{array}{r}\text { AEC-BF- } \\
\text { AEC } \\
\mu_{1}=\mathbf{0 . 1}\end{array}$ & $\begin{array}{r}\text { AEC-BF- } \\
\text { AEC } \\
\boldsymbol{\mu}_{1}=\mathbf{0 . 2}\end{array}$ & $\begin{array}{c}\text { AEC-BF- } \\
\text { AEC } \\
\boldsymbol{\mu}_{1}=\mathbf{0 . 3}\end{array}$ \\
\hline $\begin{array}{r}\text { Avg. ERLE during } \\
\text { initial steady state } \\
\text { (dB) }\end{array}$ & 7.79 & 14.82 & 14.59 & 14.19 & 13.54 & 13.05 \\
\hline $\begin{array}{c}\text { Avg. ERLE during } \\
\text { re-convergence (dB) }\end{array}$ & 6.50 & 10.40 & 10.70 & 12.07 & 12.11 & 12.09 \\
\hline $\begin{array}{c}\text { Avg. ERLE during } \\
\text { finial steady state } \\
\text { (dB) }\end{array}$ & 8.78 & 14.43 & 14.44 & 13.99 & 13.53 & 13.02 \\
\hline
\end{tabular}

TABLE 6.7 Average ERLE Performance under Real Changing Room Conditions with Increasing Front-End AEC Step Sizes for Proposed Structure

Once more, the same general trends for the average ERLE performance of all structures occurred in Table 6.7 as in Table 6.3 , with the exception of the approximately constant average ERLE performance for the proposed structure during re-convergence, as discussed in Section 6.1.1. During initial and final steady state operation the proposed AEC-BF-AEC structure's average ERLE performance was at most 0.63 to $1.77 \mathrm{~dB}$ less than the BF-AEC and AEC-BF structures as the front-end AECs step sizes increased accordingly in either case. As the front-end AECs adaptation step sizes increased the proposed structure's average ERLE performance was at least 5.26dB and 4.24dB higher than the single microphone structure during initial and final steady state operation respectively. 
During re-convergence the average ERLE value attained by the proposed structure, as the front-end AECs adaptation step sizes increased, was approximately $1.37 \mathrm{~dB}$ higher than for the BF-AEC and AEC-BF structures for all front-end AECs step sizes. The single microphone structure was outperformed by the proposed structure by about $5.57 \mathrm{~dB}$ at all front-end AECs adaptive filters step sizes, as seen in Table 6.7.

\subsubsection{Simulation Results under Increasing Tail-End AEC Step Size}

Figure 6.9, shown below, displays ERLE simulation results for all current structures and for the proposed structure where the tail-end AEC adaptation step sizes were increased from 0.1 to 0.3 while the front-end AECs adaptation step sizes were held constant at 0.15 . Also, the front-end AECs adaptive filter lengths were held constant at 80 taps. 


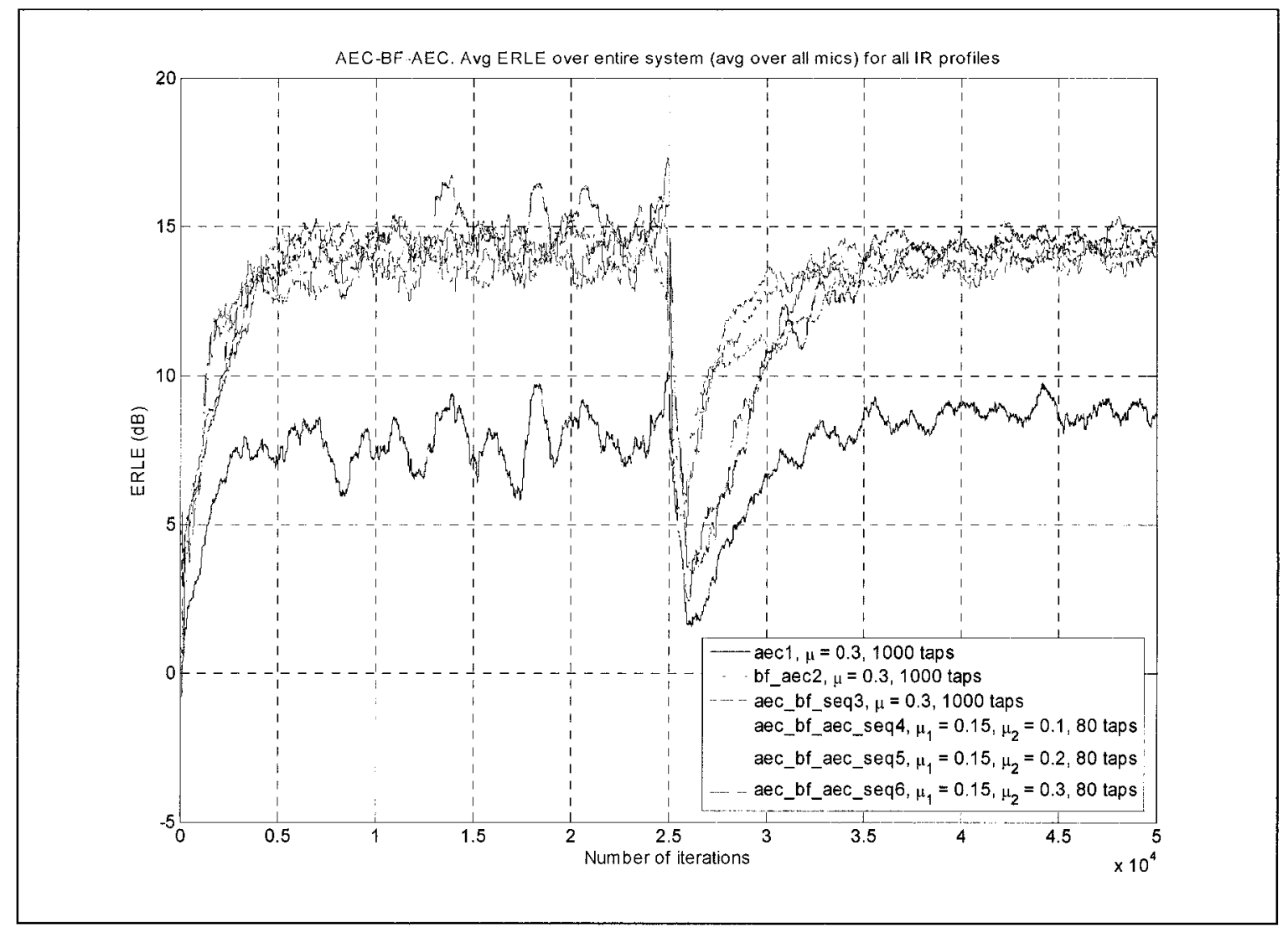

FIGURE 6.9 ERLE Results under Real Changing Room Conditions with Increasing TailEnd AEC Step Sizes for Proposed Structure

The single microphone, BF-AEC, and AEC-BF structures ERLE performance, indicated by the black, blue and red curves respectively, was the same as in Figure 6.6. Once more, the same trends occurred in the ERLE performance for the proposed structure as under the artificially changing room conditions for increasing tail-end AEC step sizes (see Figure 6.5), as discussed in Section 6.1.1. The steady state ERLE performance of the proposed AEC-BF-AEC structure slightly degraded as the tail-end AEC step size increased, as indicated by the green, cyan, and purple curves respectively. As the tail-end AEC step sizes increased the ERLE performance of the proposed structure dropped less and converged faster, but to lower steady state values, than the BF-AEC and AEC-BF structures after the change in room condition occurred. A summary of the average ERLE 
performance for all structures during initial steady state conditions, during reconvergence after the change in LRM IRs, and during final steady state conditions is given in Table 6.8 below.

\begin{tabular}{|c|c|c|c|c|c|c|}
\cline { 2 - 6 } & $\begin{array}{r}\text { AEC } \\
\text { Only }\end{array}$ & $\begin{array}{c}\text { BF- AEC- } \\
\text { AEC }\end{array}$ & $\begin{array}{r}\text { AEC-BF- } \\
\text { AEC } \\
\boldsymbol{\mu}_{2}=\mathbf{0 . 1}\end{array}$ & $\begin{array}{r}\text { AEC-BF- } \\
\text { AEC } \\
\boldsymbol{\mu}_{2}=\mathbf{0 . 2}\end{array}$ & $\begin{array}{c}\text { AEC-BF- } \\
\text { AEC } \\
\boldsymbol{\mu}_{2}=\mathbf{0 . 3}\end{array}$ \\
\hline $\begin{array}{c}\text { Avg. ERLE during } \\
\text { initial steady state } \\
\text { (dB) }\end{array}$ & 7.79 & 14.82 & 14.59 & 14.11 & 13.93 & 13.89 \\
\hline $\begin{array}{c}\text { Avg. ERLE during } \\
\text { re-convergence (dB) }\end{array}$ & 6.50 & 10.40 & 10.70 & 11.39 & 12.15 & 12.26 \\
\hline $\begin{array}{c}\text { Avg. ERLE during } \\
\text { finial steady state } \\
\text { (dB) }\end{array}$ & 8.78 & 14.43 & 14.44 & 13.95 & 13.89 & 13.92 \\
\hline
\end{tabular}

TABLE 6.8 Average ERLE Performance under Real Changing Room Conditions with Increasing Tail-End AEC Step Sizes for Proposed Structure

Again, the same general trends for the average ERLE performance of all structures occurred in Table 6.8 as in Table 6.4 as discussed in Section 6.1.1. During final steady state operation the proposed AEC-BF-AEC structure's average ERLE performance slightly decreased as the tail-end AEC step sizes increased and trailed the BF-AEC and AEC-BF structures by at most $0.55 \mathrm{~dB}$. The decrease in the proposed structure's average steady state ERLE performance was more evident during initial steady state operation as the tail-end AEC step sizes increased and trailed the BF-AEC and AEC-BF structures by no more than $0.93 \mathrm{~dB}$. As the tail-end $\mathrm{AEC}$ adaptation step sizes increased the proposed structure's average steady state ERLE performance was at least 6.1 and $5.1 \mathrm{~dB}$ higher than the single microphone structure during initial and final steady state operation respectively. 
During re-convergence the average ERLE value attained by the proposed AEC-BF-AEC structure, as the tail-end AEC adaptation step sizes increased from 0.1 to 0.3 , was between approximately 0.69 and $1.56 \mathrm{~dB}$ higher than for the BF-AEC and AEC-BF structures. The single microphone structure was outperformed by the proposed structure by about 4.89 to $5.76 \mathrm{~dB}$ as the tail-end AEC adaptive filters step sizes were increased, as seen in Table 6.8

Once more, it should be noted that the impact on average initial and final steady state ERLE performance for the proposed structure was greater when the front-end AECs step sizes were increased compared to increasing the tail-end AEC step sizes, as seen in Table 6.7 and 6.8 .

\subsubsection{Real Changing Room Environment Simulation Results Summary}

Taking the above simulation results into consideration, a summary of the trends observed on the ERLE performance of the proposed structure compared to current structures under real changing room conditions were the same as under artificially changing room conditions and are stated again for convince:

- As the length of front-end AEC adaptive filters was increased the initial and final steady state ERLE performance of the proposed structure improved towards that of the BF-AEC and AEC-BF structures and outperformed the single microphone structure by at least $4.47 \mathrm{~dB}$ (see Figures $6.6-6.7$ and Tables $6.5-6.6$ ) 
- The initial and final steady state ERLE performance of the proposed structure using relatively short front-end AEC adaptive filter lengths between 60 and 100 performed similarly to the BF-AEC and AEC-BF structures (see Figure 6.6 and Table 6.5)

- During re-convergence as the number of front-end AEC adaptive filter taps was increased between 60 and 100 the ERLE performance of the proposed structure improved and significantly outperformed the BF-AEC and AEC-BF structures by up to approximately $1.9 \mathrm{~dB}$ and the single microphone structure by at least $4.85 \mathrm{~dB}$ (see Figure 6.6 and Table 6.5)

- As the length of front-end AEC adaptive filters was increased beyond 100 taps the ERLE performance of the proposed structure, after the change in room conditions, degraded towards that of the BF-AEC and AEC-BF structures (see Figures 6.6 - 6.7 and Tables $6.5-6.6)$

- As the front-end and tail-end AEC adaptive filters step sizes were increased the initial and final steady state ERLE performance of the proposed structure degraded away from the performance of the BF-AEC and AEC-BF structures but still outperformed the single microphone structure by at least $4.24 \mathrm{~dB}$ (see Figures $6.8-6.9$ and Table $6.7)$

- During re-convergence increasing the front-end AECs adaptive filter step sizes resulted in a lower drop and faster convergence in ERLE performance for the proposed structure compared to the other structures (see Figure 6.8)

- During re-convergence increasing the tail-end AEC adaptive filter step sizes resulted in faster convergence in ERLE performance for the proposed structure compared to the other structures (see Figure 6.9) 
- Increasing the front-end AECs adaptive filters step sizes for the proposed structure had a greater negative impact on steady state ERLE performance than increasing the tail-end AEC adaptive filter step sizes (see Figures 6.8 - 6.9 and Tables 6.7 - 6.8)

- The single microphone AEC structure had the worst overall ERLE performance of all the structures (see Figures 6.6-6.9)

Once again, simulations of the proposed structure were also performed for increasing front-end and tail-end AEC adaptive filter step sizes towards and beyond 2.0. At step sizes of 2.0 and greater the proposed structure's AEC adaptive filters became divergent as discussed in Section 3.5.1.

The slightly higher average steady state ERLE values attained by either the BF-AEC or AEC-BF structures in Sections 6.1.1 and 6.1.2 compared to the other combined structures could possibly be attributed to a varying beamformer beamshape with frequency, as well as loudspeaker distortion. 


\section{CHAPTER 7 CONCLUSIONS}

This work investigated several structures for implementing hands-free communication systems using digital signal processing techniques. A new compromise structure for combining microphone array beamforming and acoustic echo cancellation for hands-free communication systems was proposed in this thesis, in an effort to offset the drawbacks of current structures under non-stationary conditions. A single microphone AEC only structure, two existing generic combined structures, and the proposed structure were investigated in this thesis. A summary of main findings of the investigations is presented in the following section.

\subsection{Summary of Results}

As a result of performing the LRM IR experiments, discussed in Chapter 4, trends were observed in the dynamic behaviour of the office room acoustics as discussed in Section 4.4.2. Specifically, the first several taps of each LRM IR remained fairly constant while the vast majority of changes in the LRM IRs occurred in roughly the next hundred taps. The rest of the LRM IR taps remained relatively unchanged.

The proposed AEC-BF-AEC structure (see Section 3.4) along with the single microphone structure (see Section 3.1), as well as the existing BF-AEC (see Section 3.2) and AEC-BF (see Section 3.3) structures were studied under stationary room environment and beamforming conditions (see Section 5.2.1). Under these conditions the proposed structure's ERLE performance using 70 front-end AEC adaptive filter taps was 
approximately the same as the BF-AEC and AEC-BF structures. Thus a major computational savings was realized as the reduced complexity proposed structure was able to perform at an almost equivalent level to the highly complex AEC-BF structure. As the number of front-end AEC taps increased beyond 70 for the proposed structure only a marginal increase in ERLE performance occurred. Also, the proposed structure's ERLE performance was at least $4 \mathrm{~dB}$ greater than that of the single microphone structure when at least 40 front-end AEC adaptive filter taps were used in the proposed structure (see Section 5.2.1).

Under the stationary room and changing BF conditions discussed in Section 5.3.1 the proposed AEC-BF-AEC structure's steady state ERLE performance was about the same as the $\mathrm{AEC}-\mathrm{BF}$ and $\mathrm{BF}-\mathrm{AEC}$ structures when relatively short front-end $\mathrm{AEC}$ adaptive filters between 60 and 100 taps were used. Again, the proposed structure's steady state ERLE performance was greater than that of the single microphone structure by at least $5.12 \mathrm{~dB}$ when more than 60 front-end AEC taps were used in the proposed structure. After the BF sector switch occurred the BF-AEC structure's ERLE performance was severely impacted but the proposed structure was only slightly affected by the BF time variations at all front-end AEC filter lengths as shown in Section 5.3.1. During the reconvergence period the proposed structure outperformed the BF-AEC structure by at least $1.12 \mathrm{~dB}$ on average. The AEC-BF structure was unaffected by the BF sector switch.

Operating under changing room and fixed BF conditions the proposed structure was able to attain very similar steady state ERLE performance as the BF-AEC and AEC-BF 
structures using small front-end AEC adaptive filters up to 100 taps as discussed in Sections 6.1.1 and 6.1.2. The proposed structure's steady state ERLE performance was at least $4.47 \mathrm{~dB}$ greater than that of the single microphone structure for both changing room conditions. After the change in the room environment occurred the BF-AEC and AECBF structures ERLE performance was significantly impaired but the proposed structure's ERLE performance was not impacted as much when relatively short front-end AEC adaptive filters up to 100 taps were used, as seen in Sections 6.1.1 and 6.1.2. During the re-convergence period the proposed structure outperformed the BF-AEC and AEC-BF structures by up to $5 \mathrm{~dB}$ and the single microphone structure by at least $7 \mathrm{~dB}$ on average under the artificially changing room conditions of Section 6.1.1. Under the real changing room conditions of Section 6.1.2 the proposed structure outperformed the current combined structures by up to $1.9 \mathrm{~dB}$ and the single microphone structure by at least $4.85 \mathrm{~dB}$ on average during the re-convergence period. As the length of the front-end AEC adaptive filters increased beyond 100 taps the ERLE performance of the proposed structure during re-convergence began to suffer more and approached that of the BF-AEC and AEC-BF structures under both changing room conditions.

Overall the proposed structure was able to perform approximately as well as the BF-AEC and AEC-BF structures under stationary conditions while requiring significantly less computational resources than the AEC-BF structure. Although the proposed structure remained more complex than the $\mathrm{BF}-\mathrm{AEC}$ structure it was more robust than the $\mathrm{BF}-\mathrm{AEC}$ structure to changing $\mathrm{BF}$ and room conditions. The proposed structure was also more robust than the AEC-BF structure to changing room conditions. 


\subsection{Recommendations for Future Research}

As a result of the studies performed in this thesis there are several areas in which research could continue. The structures could be reevaluated using speech signals as the far-end input instead of WGN. This would verify the performance of the proposed structure under more realistic hands-free communication system conditions, to see if similar results to the ones found in this work could be achieved.

Also, the hands-free communication system structures investigated in this thesis could be implemented in real-time on DSP hardware. This would allow the performance of all structures to be studied and verified in a real world hand-free communication system. If the structures were to be implemented on fixed-point DSP hardware, instead of floatingpoint, then care must be taken to ensure that the underlying structure algorithms are not affected by the numerical limitations of the fixed-point hardware.

Another source of future research would be to investigate the feasibility of using more elaborate algorithms for AEC, such as RLS type adaptive filtering algorithms or the FAP algorithm. Also, subband or transform domain adaptive filtering approaches, as well as the possibility of using adaptive beamforming algorithms could be investigated. Using more elaborate algorithms could result in a hands-free communication system structure that achieves superior results compared to the structure proposed in this thesis. 


\section{REFERENCES}

[1] D. G. Messerschmitt, "Echo cancellation in speech and data transmission," IEEE J. Select. Areas Commun., vol. SAC-2, pp. 283-297, Mar. 1984.

[2] K. Murano, S. Unagami, and F. Amano, "Echo cancellation and applications," IEEE Commun. Mag., vol. 28, pp. 49-55, Jan. 1990.

[3] M. M. Sondhi and D. A. Berkeley, "Silencing echoes in the telephone network," Proc. IEEE, vol. 68, pp. 948-963, Aug. 1980.

[4] M. M. Sondhi and W. Kellermann, "Adaptive echo cancellation for speech signal," Advances in Speech Signal Processing, S. Furui and M. Sondhi, Ed. New York: Dekker, ch. 11, 1992.

[5] M. E. Knappe, "Acoustic Echo Cancellation: Performance and Structures," M.Eng Thesis, Carleton University, Ottawa, Canada, July 1992.

[6] M. Dahl and I. Claesson, "Acoustic noise and echo canceling with microphone array," IEEE Trans. Veh. Technol., vol. 48, pp. 1518-1526, Sept. 1999.

[7] M. Kallinger, J. Bitzer, and K.-D. Kammeyer, "Study on combining multichannel echo cancellers with beamformers," in Proc. IEEE ICASSP, vol. 2, pp. 797-800, Jun. 2000.

[8] H. Buchner, W. Herbordt, and W. Kellermann, "An Efficient Combination of Multi-Channel Acoustic Echo Cancellation With a Beamforming Microphone Array," in Proc. Int. Workshop on Hands-Free Speech Communication (HSC), pp. 55-58, Kyoto, Japan, April, 2001.

[9] W. Herbordt, H. Buchner, and W. Kellermann, "Computationally Efficient Frequency-Domain Combination of Acoustic Echo Cancellation and Robust Adaptive Beamforming," in Proc. EUROSPEECH, vol. 2, pp. 1001-1004, Aalborg, Danmark, September, 2001.

[10] W. Herbordt, and W. Kellermann, "Limits for generalized sidelobe cancellers with embedded acoustic echo cancellation," in Proc. IEEE ICASSP, vol. 5, pp. 3241-3244, May 2001.

[11] M. Dahl, I. Claesson, and S. Nordebo, "Simultaneous echo cancellation and car noise suppression employing a microphone array," in Proc. IEEE ICASSP, vol. 1 pp. 239-242, Apr. 1997. 
[12] W. Kellermann, "Strategies for combining acoustic echo cancellation and adaptive beamforming microphone arrays," in Proc. IEEE ICASSP, 1997, pp. 219-222.

[13] W. L. Kellermann, "Acoustic echo cancellation for beamforming microphone arrays," in Microphone Arrays: Signal Processing Techniques and Applications, M. Brandstein, and D. Ward, Eds. Berlin: Springer, 2001, pp. 281-306

[14] M.J. Reed, and M.O.J. Hawksford, "Acoustic echo cancellation with the fast affine projection," in IEE Colloquium on Audio and Music Tech., pp. 16/1-16/8, Nov. 1998.

[15] M. Montazeri and P. Duhamel, "A set of algorithms linking NLMS and block RLS algorithms," IEEE Trans. Signal Processing, vol. 43, pp. 444-453, Feb. 1995.

[16] M. Tahernezhadi, J. Liu, G. Miller, and X. Kong, "Acoustic echo cancellation using subband technique for teleconferencing applications," IEEE Global Telecomm. Conf., vol. 1, pp. 243-247, Dec. 1994.

[17] S. Gudvangen, and S.J. Flockton, "Intermediate complexity adaptive filters in acoustic echo cancellation," in IEE Colloquium on New Directions in Adaptive Signal Processing, pp. 5/1-5/4, Feb. 1993.

[18] P. S. R. Diniz, Adaptive Filtering: Algorithms and Implementation. Boston, MA: Kluwer Academic Publishers, 1997.

[19] S. Haykin, Adaptive Filter Theory, $3^{\text {rd }}$ ed., Upper Saddle River, NJ: Prentice-Hall, 1996.

[20] M. Tahernezhadi, and M. Dai, "Application of QR-LS algorithm for one-channel and two-channel acoustic echo cancellation," in IEEE $39^{\text {th }}$ Midwest symposium on Circuits and Systems, vol. 2 pp. 813-816, Aug. 1996.

[21] M. G. Siqueira, and A. Alwan, "New adaptive-filtering techniques applied to speech echo cancellation," in Proc. IEEE ICASSP, vol. 2, pp. 265-268, Apr. 1994.

[22] F.Capman, J.Boudy, and P.Lockwood, "Acoustic echo cancellation using a fast QR-RLS algorithm and multirate schemes," in Proc. IEEE ICASSP, vol. 2, pp. 969-972, May 1995.

[23] T. Petillon, A. Gilloire, and S. Theodoridis, "Complexity reduction in fast RLS transversal adaptive filters with application to acoustic echo cancellation," in Proc. IEEE ICASSP, vol. 4, pp. 37-40, Mar. 1992. 
[24] S.L. Gay, "Dynamically regularized fast RLS with application to echo cancellation," in Proc. IEEE ICASSP, vol. 2, pp. 957-960, May 1996.

[25] W.Kellermann, "Analysis and design of multirate systems for cancellation of acoustical echoes," in Proc. ICASSP, 1988, pp. 2570-2573.

[26] A. Gilloire and M. Vetterli, "Adaptive filtering in subbands with critical sampling: Analysis, experiments, and application to acoustic echo cancellation," IEEE Trans. Signal Processing, vol. 40, pp. 1862-1875, Aug. 1992.

[27] J.Benesty and D. R.Morgan, "Frequency-domain adaptive filtering revisited, generalization to the multi-channel case, and application to acoustic echo cancellation," in Proc. IEEE ICASSP, vol. 2, 2000, pp. 789-792.

[28] A.Gilloire and M.Vetterli, "Adaptive filtering in sub-bands," in Proc. IEEE ICASSP, vol. 3, pp. 1572-1575, Apr. 1988.

[29] Y. Bendel, D. Burshtein, O. Shalvi, and E. Weinstein, "Delayless frequency domain acoustic echo cancellation," in IEEE Trans. Speech Audio Processing, vol. 9, pp. 589-597, July 2001.

[30] O.A. Amrane, E. Moulines, M. Charbit, and Y. Grenier, "Low-delay frequency domain LMS algorithm," in Proc. IEEE ICASSP, vol. 4, pp. 9-12, Mar. 1992.

[31] S.L.Gay and S.Tavathia, "The fast affine projection algorithm," in Proc. IEEE ICASSP, vol. 5, pp. 3023-3026, May 1995.

[32] R. H. Kwong and E. W. Johnston, "A variable step size LMS algorithm," IEEE Trans. Signal Processing, vol. 40, pp. 1633-1642, July 1992.

[33] V. J. Mathews and Z. Xie, "A stochastic gradient adaptive filter with gradient adaptive step size," IEEE Trans. Signal Processing, vol. 41, pp. 2075-2087, June 1993.

[34] J. B. Evans, P. Xue, and B. Liu, "Analysis and implementation of variable step size adaptive algorithms," IEEE Trans. Signal Processing, vol. 41, pp. 25172535, Aug. 1993.

[35] E. Jan and J. Flanagan, "Microphone arrays for speech processing," in Proc. IEEE ISSSE, 1995, pp. 373-376.

[36] B.D. Van Veen, and K.M. Buckley, "Beamforming: A versatile approach to spatial filtering," IEEE ASSP Magazine, vol. 5, pp. 4-24, Apr. 1988. 
[37] J.G. Ryan, and R.A. Goubran, "Application of near-field optimum microphone arrays to hands-free mobile telephony," IEEE Trans. Veh. Technol., vol. 52, pp. 390-400, Mar. 2003.

[38] S. Oh, V. Viswanathan, and P. Panamichalis, "Hands-free voice communication in an automobile with a microphone array," in Proc. IEEE ICASSP, vol. 1, pp. 281-284, Mar. 1992.

[39] Y. Grenier, "A microphone array for car environments," in Proc. IEEE ICASSP, vol. 1, pp. 305-308, Mar. 1992.

[40] B.D.Van Veen, "An analysis of several partially adaptive beamformer designs," IEEE Trans. Acoust., Speech, Signal Processing, vol. 37, pp. 192-203, Feb. 1989.

[41] H. Yang and M.A. Ingram, "Design of partially-adaptive arrays using the singular value decomposition," IEEE Trans. Antennas Propagat., vol. 45, pp. 843-850, May 1997.

[42] W.H. Neo and B. Farhang-Boroujeny, "Robust microphone arrays using subband adaptive filters," in Proc. IEEE ICASSP, vol. 6, pp. 3721-3724, May 2001.

[43] S.E. Nordholm, I. Claesson, and N. Grbic, "Performance limits in subband beamforming," in IEEE Trans. Speech Audio Processing, vol. 11, pp. 193-203, May 2003.

[44] S.Nordholm, I.Claesson, and M.Dahl, "Adaptive microphone array employing calibration signals: An analytical evaluation," IEEE Trans. Speech Audio Processing, vol. 7, pp. 241-252, May 1999.

[45] S. Doclo, M. Moonen, and E. de Clippel, "Combined acoustic echo and noise reduction using GSVD-based optimal filtering," in Proc. IEEE ICASSP, vol. 2, pp. 1061-1064, Jun. 2000.

[46] J. Lariviere, and R. Goubran, "GMDF for noise reduction and echo cancellation," IEEE Signal Processing Letters, vol. 7, pp. 230-232, Aug. 2000.

[47] W.L.B. Jeannes, P. Scalart, G. Faucon, and C. Beaugeant, "Combined noise and echo reduction in hands-free systems: a survey," IEEE Trans. Speech Audio Processing, vol. 9, pp. 808-820, Nov. 2001.

[48] A. N. Birkett and R. Goubran, "Acoustic echo cancellation using NLMS-neural network structures," in Proc. IEEE ICASSP, vol. 5, pp. 3035-3038, May 1995.

[49] F. Kuch, and W. Kellermann, "Nonlinear line echo cancellation using a simplified second order Volterra filter," in Proc. IEEE ICASSP, vol. 2, pp. 1117-1120, 2002. 
[50] J. Chen and J. Vandewalle, "Study of adaptive nonlinear echo canceller with volterra expansion," in Proc. IEEE ICASSP, vol. 2, pp. 1376-1379, May 1989.

[51] G. Sicuranza, A. Bucconi, and P. Mitri, "Adaptive echo cancellation with nonlinear digital filters," in Proc. IEEE ICASSP, vol. 9, pp. 130-133, Mar. 1984.

[52] H. Yuan, "Dynamic Behavior of Acoustic Echo Cancellation," M.Eng Thesis, Carleton University, Ottawa, Canada, January 1994.

[53] D.H. Johnson, and D.E. Dudgeon, Array Signal Processing: Concepts and Techniques. Englewood Cliffs, NJ: Prentice-Hall, 1993. 